\begin{tabular}{ll||}
\hline B & \\
205 & $\$ C 164846$
\end{tabular}

$45 \mathrm{AS}$

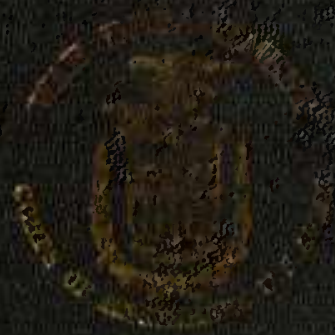

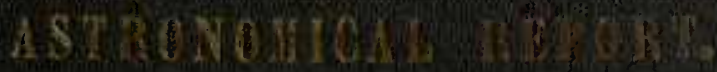

GWOCRARUCAT AND CGOMOCOAL

BXPLORATLWS ANE STRWDS

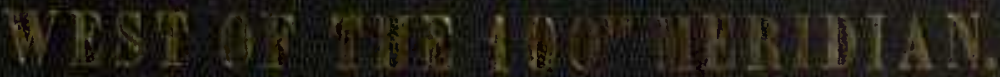

1.8\% 2. 
GIBIATE

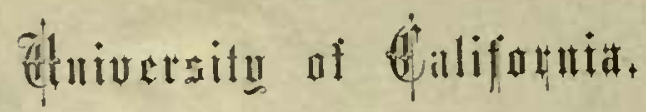

No.

42.

Division

Range

Shelf

Received 1874.

Wrath the Complimensis of

$r$

Bearge Ollo. Orhecles,

cinst Lient. Couphe of Bingineew,

Q6. D) dump 






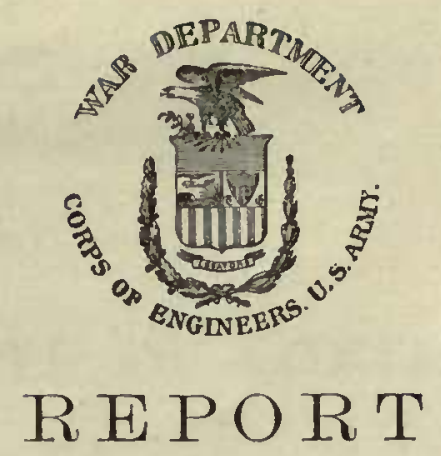

UPON

\section{THE DE'TERMINATION}

OF TIIE

\section{.ASTRONOMICAL CO-ORDINATES}

OF

THE PRINARY STATIONS AT CHEYENNE, WYOMING TERRITORY, AND COLORADO SPRINGS, COLORADO TERRITORY,

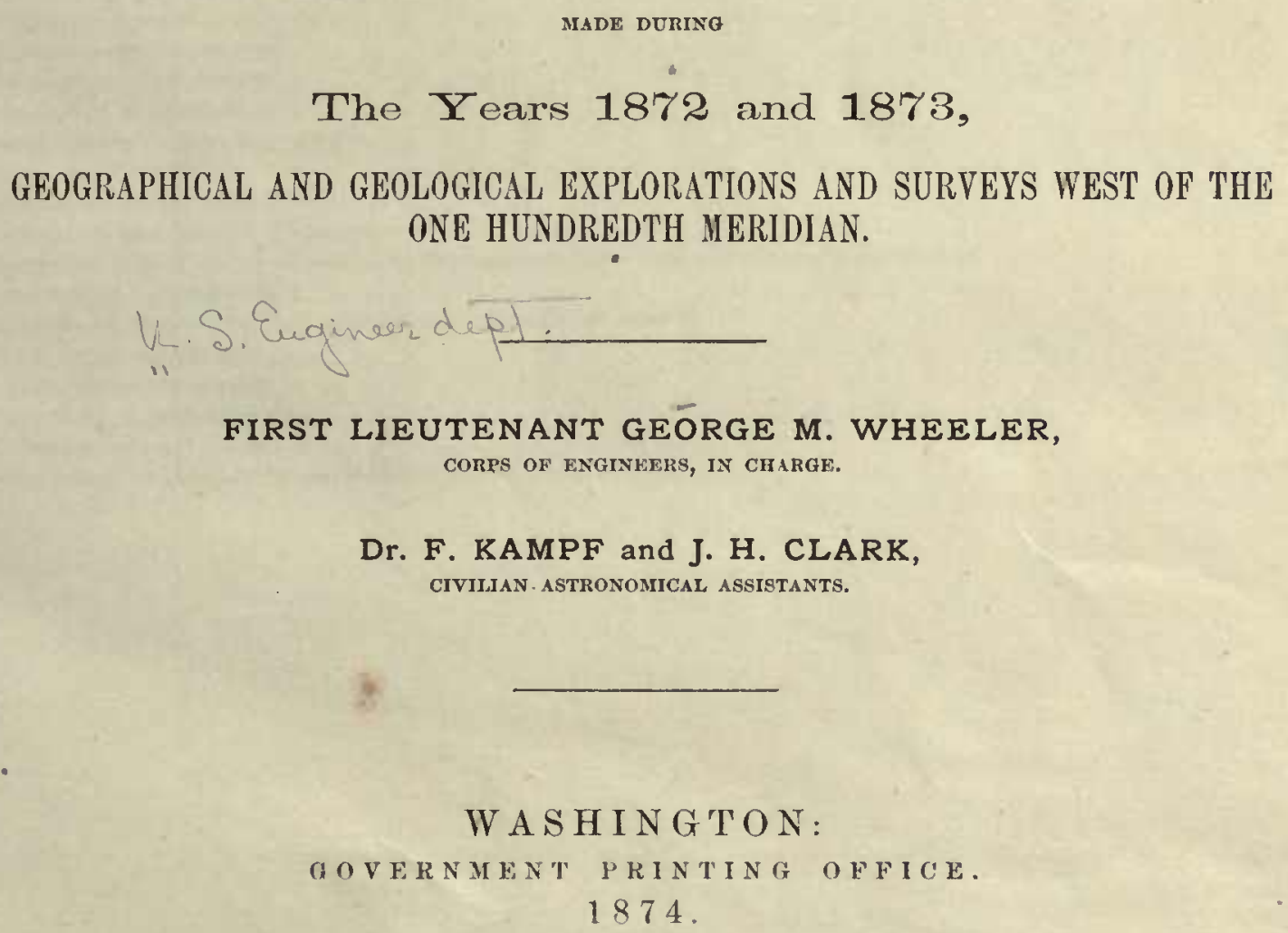





\section{TABLE OF CONTENTS.}

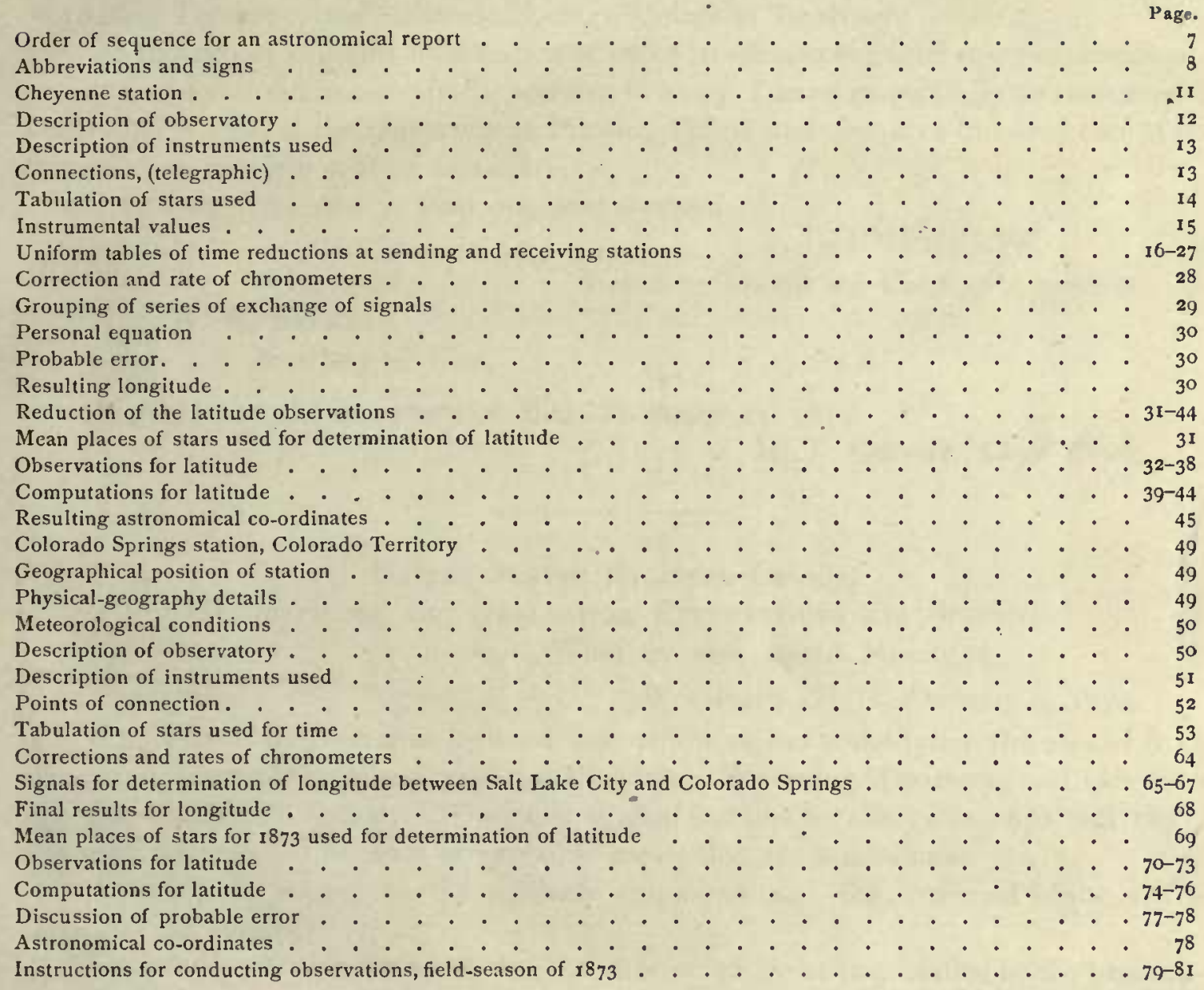





\section{Office of the Chimp of Evaneers, \\ Washington, D. C., February 13,1874 .}

Sir: Lient. George M. Wheeler, Corps of Engineers, has sent to this office a report embodying the results from the astronomical observations made at Cheyenne, Wyoming Territory, and Colorado Springs, Colorado Territory.

As this report contains information of value to officers engaged upon explorations, surveys, and reconnaissances in the westerm country, I have respectfully to recommend that it be printed at the Government Printing-Office, and that five hundred copies be furnished on requisition from this office.

Very respectfully, your obedient servant,

A. A. HUMPHREYS, Brigadier-General and Chief of Engineers.

Hon. W. W. BeLKNAP, Secretary of War.

Approved by the Secretary of War, February 14, 1874.

H. T. Crossy, Chief Clerk.

\section{United States Engineer Office, Geograpitical and Geologrcal Explorations and Surveys \\ West of the iootil Mermian, Washington, D. C., February 9, 1874.}

SIR: I have the honor to forward herewith a report embodying the results from the astronomical observations made at Cheyenne, Wyoming Territory, and Colorado Springs, Colorado Territory. They are typical stations for the years 1872 and 1873 , although not selected because of probable errors that are a minimum.

Attention is invited to the methods employed and the order of sequence in reporting the results.

Uniformity of plan seems to be a matter of so great importance in the prosecution of astronomical work in the western interior that the one now in use is submitted for consideration as a step at least in this direction.

Minor features of the methods are still to be perfected; yet the errors of starplaces, from observation, and those known as instrumental, have now become reduced to such small and nearly equable values, that it seems desirable that some general and uniform plan should be adopted for the report at least, if not for the nanner of conducting the observations.

The present one is submitted with extreme diffidence, and will probably demand certain ehanges in the light of future experience.

At Colorado Springs a heavy stone monument and observing-pier, with meridianmarks, have been establisher. 
The same has been done at the greater number of the other points occupied as main field-stations in the years 1871,1872 , and 1873 .

These meridian-lines may serve various purposes in clıcking future surveys in adjacent areas.

Very respectfully, your obedient servant,

GEO. M. WHEELER,

Brig. Gen. A. A. Humphreys,

Lieutenant of Engineers, in Charge.

Chief of Engineers, United States Army.

Note.-The results from the observations made during the field-seasons of $187 \mathrm{r}$, 1872 , and 1873 , at the remaining primary stations, twenty-two in number, will appear in Volume II of the Reports of the Survey, as proposed in the Annual Report of the Chief of Engineers for I873, Appendix EE. 
ORDER OF SEQUENCE

FOR AN

\section{A S T R O N O M I C.A L R E P O R T}

AT

MAIN OR PRIMARY FIELD-STATIONS.

Longitude by Telegraph; Latitude by Zenith Telescope, (Talcott's method.)

I. Goographical position of station.

2. Plyysical-geography details; especially all physical peculiarities.

3. Meteorological conditions, both general and special; the latter while observations were made.

4. Description of observatory; including personnel of party, name of telegraphoperator, and name of telegraph-company whose wire has been employed.

5. Description of instruments used.

6. Points witl which connections were made; nights of observation, and observers; also name of computer or computers.

7. Tabulation of stars used, and number of observations.

8. Instrumental values; circumstances of telegraphic communication, $i$. e., length of circuit, number of batteries, repeaters, \&c.

9. Uniform tables of time-reductions at recoiving-station.

10. Uniform tables of time-reductions at sending-station.

I I. Grouping of series of excliange-signals, including means of single and serial results.

I 2. Personal equation.

13. Probable error by least squares.

14. Resulting longitude.

I5. Reduction of the latitude-observations properly grouped, with discussion of results.

I6. Resulting astronomical co-ordinates. 


\section{ABBREVIATIONS AND SIGNS.}

$a, b, c,=$ azimuth, level, and collimation corrections.

$\mathrm{A}, \mathrm{B}, \mathrm{C},=$ azimuth, level, and collimation factors.

$\mathrm{T}=$ observed time reduced to the mean of wires and corrected for rate.

$\mathrm{T}^{\prime}=$ observed time corrected for instrumental errors.

$\mathrm{AR} .=$ apparent right ascension of star.

$\Delta_{0} \mathrm{~T}=$ resulting error of the chronometer after the mean of the wires is corrected for rate and level.

$\Delta \mathrm{T}_{0}=$ adopted mean error of chronometer.

$\delta \mathrm{T}=\Delta \mathrm{T}_{0}-\Delta_{0} \mathrm{~T}$.

$\Delta \mathrm{T}=$ error of the chronometer.

$v=$ difference between mean final correction of chronometer and $\Delta \mathrm{T}$. 


\section{R E P O R T \\ ON \\ ASTRONOMICAL OPERATIONS, \\ CONDUCTED DURING}

\section{THE FIELD-SEASON OF 1872,}

AT THE

MAIN OR PRIMARY FIELD-STATION, CHEYENNE, WYOMING TERRITORY,

AND

DEDUCTION OF RESULTS.

BY

DR. F. KAMPF AND J. H. CLARK, CIVTLIAN ASTRONOMICAL ABSISTANTS, 


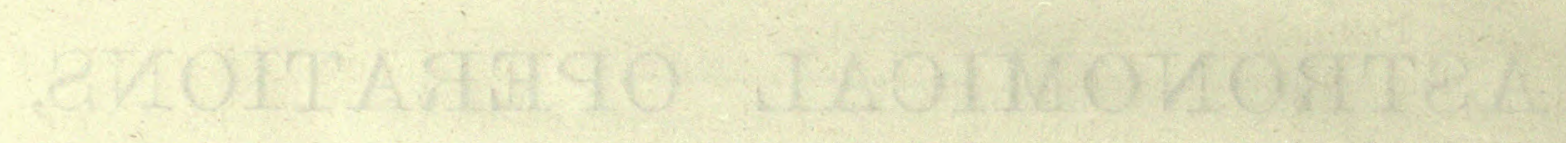




\section{United States EngineER Office, Geograpilical and Geological Explorations and Surveys West of true rooth Meridian, Washington, D. C., Jamuary I, 1874.}

\section{CHEYENNE, WYOMING TERRITORY.}

\section{(i.) Geographical Position of Station.}

The station at Cheyenne, the latitude of which is N. $41^{\circ} 7^{\prime} 46^{\prime \prime} .62$, and its longitude $28^{\mathrm{m}} 19^{\mathrm{s}} .44$ east of the Mormon observatory at Salt Lake, is situated in the western part of the town, in lot II, block $4 \mathrm{I} 3$, and is nortliwest from the junction of the branchroad leading to the depot of Fort D. A. Rirssell with the main stem of the Union Pacific Railroad.

The town of Cheyenne, on the north side of the railroad, is the capital of Wyoming Territory; and besides the Union Pacific Railroad with its branch to Fort Russell, has a railroad-connection with Denver, Colorado. These facilities make it a flourishing place, and it possesses already, in addition to its public buildings, quite a number of substantial business-houses and attractive private residences.

A fine hotel, engine-houses, workshops, and other similar improvements, are among the sources of prosperity which spring directly from the railroad-company.

Besides the traffic with Fort Russell, it is the depot for Red Cloud's agency, Fort Laramie, and the settlements on the North Platte.

The surrounding region affords unlimited pasturage, and cattle are said not only to subsist but grow fat on it during the whole year:

Agriculturally there is but little promise outside of what may be accomplished by irrigation.

\section{(2.) Physical-Geography Details.}

The site of Cheyenne is nearly or quite level; northward, however, there is a gradual swell of the land, and within a fer miles it cuts of the extensive view one is accustomed to on the great plains. Eastward and southward there is the usual rolling and slightly-broken prairie; but in the southwest, Long's Peak, already white this September with snow, some seventy miles distant, looms up boldly above the horizon. West and northwest, low mountains, many of which mark the rim of the Laramie Plains, are just visible. Crow Creek, a moderate stream coming down from the foothills of this rim, forms a valley immediately west and south of the town, working its way apparently through mountain-drift, as the direct eruptive force of the Rocky Mountain system seems not to have reached so far east in this latitude.

\section{(3.) Meteorological Conditions.}

The determination of this station occupied the first three weeks in the month of October, 1872. There were no rains, nor any clonds, and, with the exception of two or three windy nights, the elements presented no obstacle whatever to a continued series of observations. In the early morning it would become cold enough to form 
ice, making it sufficiently uncomfortable to restrain the ardor of an observer from extending his observations into the small hours of the night. From the experience of this season, and that of many others which I have undergone in former years in astronomical work, both on the plains and in the mountains, I am of the opinion that the best time of the year (so far as the weather is concerned) for astronomical observations in the Territories and the far Western States occurs in September and October. They are the months that fall between the rainy season of the summer' and the stormy weather of the winter.

The following table shows the direction of the wind, and the estimated force, from 7 a. m. to 7 p. m. and from 7 p. m. to 7 a. m. The weather was clear all the time. Such an atmosphere, at an elevation of 6,000 feet, could not be othervise than favorable for astronomical work.

\begin{tabular}{|c|c|c|c|c|c|}
\hline \multirow{2}{*}{ Date. } & \multicolumn{2}{|c|}{ General direction of wind. } & \multicolumn{2}{|c|}{ Estimated force of wlnd, } & \multirow{2}{*}{ Remarks. } \\
\hline & 7 a. m. to 7 p. m. & 7 p. m. 107 a. m. & 7 a. m. to 7 p. m. & 7 p. m. to 7 a. m. & \\
\hline 1872. & $311=48$ & 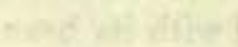 & 20 & 77 & \\
\hline October 5 & NW. & N. & I & I & \\
\hline 6 & sw. & o & I & o & \\
\hline 7 & NW. & o & t & o & \\
\hline 8 & NE. & o & $\mathrm{I}$ & 。 & \\
\hline 9 & SE. & o & I & tht & . \\
\hline 10 & SE. & 0 & I & o & \\
\hline II & N. & o & I & o & \\
\hline 12 & N. & o & I & o & \\
\hline 13 & o & 0 & o & o & \\
\hline 14 & NW. & NW. & 1 & I & Aurora borealis in the north \\
\hline 15 & NW. & o & 2 & o & at $\mathrm{It} .30 \mathrm{p} . \mathrm{m}$. \\
\hline 16 & NW. & o & (⿻)丨 & o & \\
\hline 17 & sw. & a & I & o & Aurora borcalis in the north \\
\hline 18 & o & o & 0 & o & \\
\hline 19 & w. & o & I & o & \\
\hline 20 & w. & N. & I & 2 & \\
\hline
\end{tabular}

(4.) Description of Observatory.

A large wall-tent drawn over a framework formed the observatory. The opening for the meridian-line was furnished with a flap and curtains; the former served to protect the instrument from the weather when not in use, and the latter to keep off the wind and dust while observing. Here, and at other stations previously occupied, the opening for the meridian-line in the observatory was entirely nnobstructed. Subsequently it was found to be an improvement to retain the ridge-pole (which commonly interferes but little with the completeness of the observations) as a support against the violent winds so prevalent in the conntry in which our operations are conducted. The tent was furnished with all the appliances of a field and temporary observatory, such as stools, stands, tables, and the like, and nothing was wanting as to equipment for firstclass field-work. The Western Union Telegraph Company furnished the line and other facilities for this station. Mr. Bates, one of the operators employed at the Cheyenine oflice, was assigned to do the telegraphing; and when business did not per- 
mit him to leave, which sometimes happened, Mr. Henderson, who was off duty at this lour, was obliging enough to supply his place gratuitously. Mr. F. R. Simonton was my assistant here, and kept, in conjunction with C. Herbert, an hourly meteorological record, besides lis other duties as assistant.

\section{(5.) Description of Instruments Used.}

\section{(a.) Transit.}

The transit used was the meridian-zenith-instrument No. 28, made by Wuirdemann, a description of which is given in Dr. Kampf's report, and it was mounted on a large block of wood. 'The instiumental values are, for one division of the micrometerscrew, $\mathrm{O}^{\prime \prime} .6216$; of the striding-level A, I".2I; and of the zenith-level, $\mathrm{I}^{\prime \prime} .10$.

\section{(b.) Clironograph.}

The record of the observations for time, as well as the exchange of signals, with one exception (the night of the I 4 th ), was made by means of the chronograph, of the form contrived by Professor Harkness, United States Naval Observatory. It consists of clock-work driven by a weight, and ean be adjusted to run some two lours. The regulation of the movement is effected by a steel spring, with movable balances, striking on a fly-wheel. A cylinder is attached, covered with paper, and is made to revolve once a minute. Along this cylinder a screw carries a pen, which, being in the same circuit with the chronometer, records its breaks. The chronometric breaks are made every second, except the sixtieth, which is omitted, to mark the minute. Removing the paper from the cylinder, both the minutes and the seconds will be found, if the instrument is working properly, recorded in parallel lines, and the culminations of the stars observed, distinctly marked by arbitrary breaks, and easily read off.

\section{(c.) Chronométer.}

The chronometer in use here was the Negus break-circuit No. r499. It had a gaining-rate, +0.054 , hourly average, at a mean temperature of $50^{\circ}$. The breakarrangement got out of order once, but it was readily repaired, and ran the rest of the season without giving any trouble.

\section{(d.) Battery.}

A local circuit of sufficient force was produced by two cups of zinc and copper, a form known, I believe, among electricians as the Hill battery. It is sinple, works a week or more without renewal, and the only possible objection to it for the purpose to which we apply it is that the sulphate is a little slow to act, particularly in cold weather.

\section{(6.) Connections.}

The observatory was west of the telegrapli-office, and the main connection was effected by a loop into one of the main overland wires and put in communication with Salt Lake by a sivitch at Ogden. The local connections, including the chronometer, the cluronograph, and the observing-keys, were made by means of a switch-board, which also received the main circuit. 'The various wires being put in their proper 
posts on this board, and the circuit closer, it required only the simple movement of sliding a switch-button to cut off or put on either circuit, and thus send the clironometric break, or receive that of the connected station on the chronographic sheet, as desired. The nights of the Ist, $3 \mathrm{~d}, 8 \mathrm{th}$, 9 th, Ioth, 11 th, and 1 2th were occupied on latitude, in which time 257 results were obtained. The 5th, 14th, I6th, I 8th, 19th, and 2 Ist were successively put in on longitude. 'T'here were several other evenings when efforts were made in this direction, but failed from causes beyond my control. Mr. Austin did the observing at Salt Lake, while the observations at Cheyenne were made by myself, and also the longitude reductions for both stations. Prof. W. A. Rogers, of Harvard College Observatory, computed the latitude-observations, and they were subsequently revised, partially recomputed, and formulated by Dr. Kampf.

\section{(7.) Tabulation of Stars Used.}

Tabulation of Stars used for Determination of Time at Cheyenne, Wyoming Territory, and Salt Lake City, Utah Tervitory.

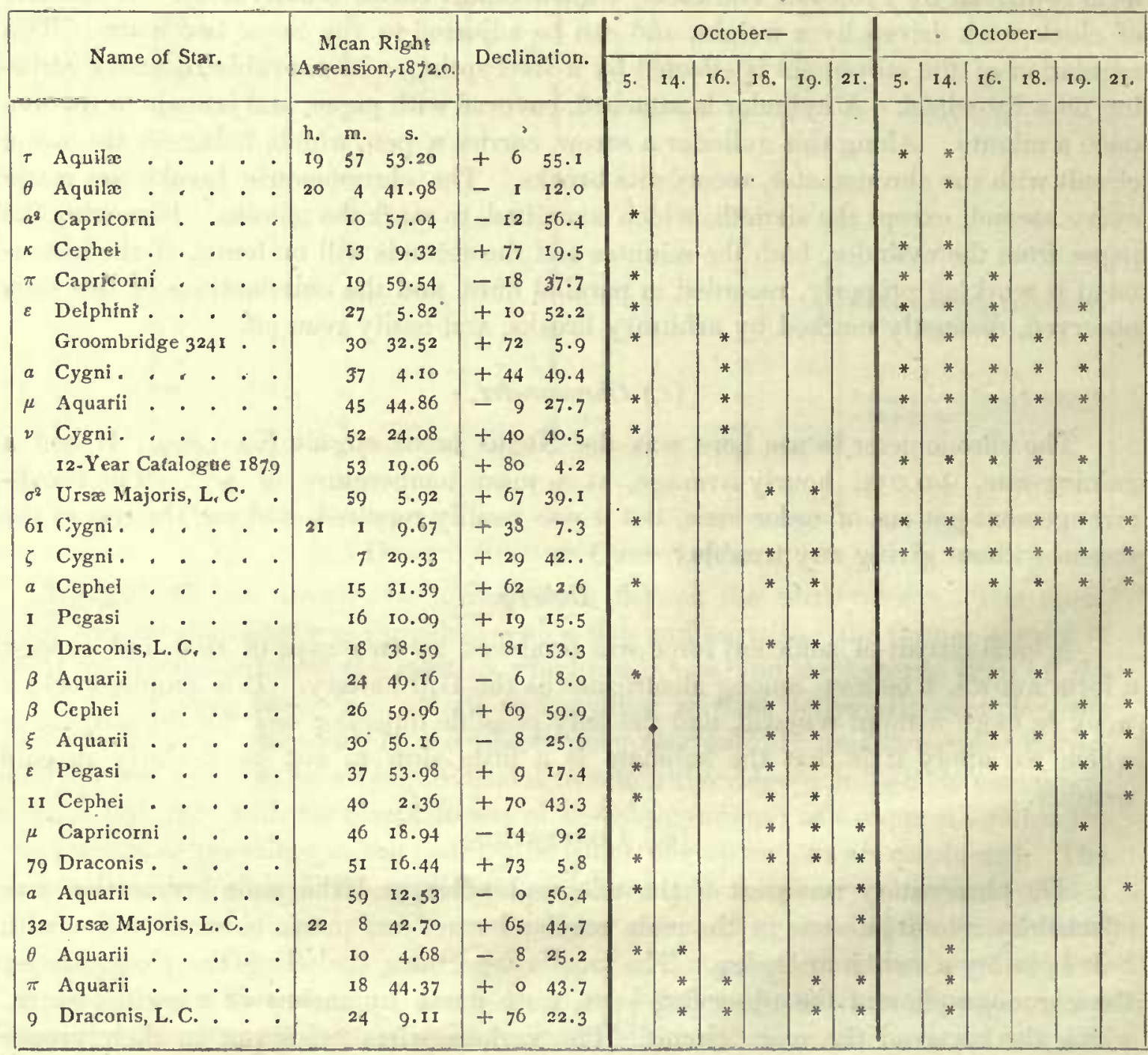


Tabulation of Stars used for Determination of Time, \&c.-Continued.

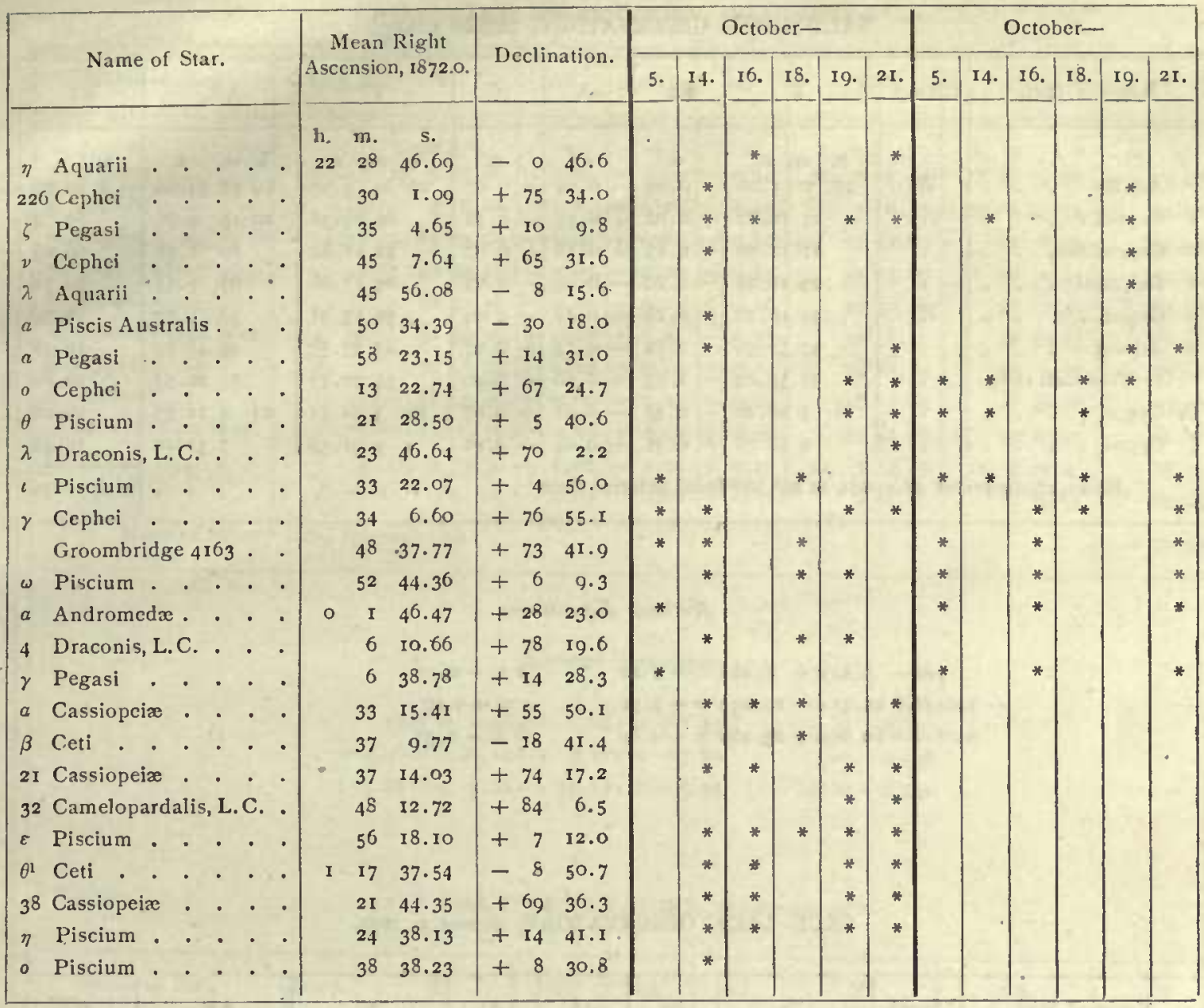

\section{(8.) Instrumental Values.}

The values of the implements pertaining to zenith-instrument No. 28 are, for one division of the micrometer-screw, $\mathrm{O}^{\prime \prime} .622$; of the striding-level $\mathrm{A}, \mathrm{I}^{\prime \prime} .21$; and of the zenith-level, I".10. The wire which made the circuit between the stations Salt Lake and Cheyenne, counting the distance along the railroad, is some 550 miles, and does not include any battery or repeating-office. At Cheyenne there was a repenting-office; but as the observatory, as elsewhere stated, was situated west of it, the signals did not work through any repenter; and at the time these observations were made Salt Lake itself was only a relay-office; but there was a switch at Ogden, which had always to be called to "straighten" the wire before the work of exchanging could be commenced. Powerful batteries have always been kept at both places, and lately the repeating-office at Corinne has been removed to Salt Lake, and the latter has not only become a repeating-office but also the headquarters of the western division of the main or eastern branch of the company. 
(9:) Uniform 'Tables of Trme-Reducrions at Receiving-Station.

SALT LAKE OBSERVATORY, October 5, 1872.

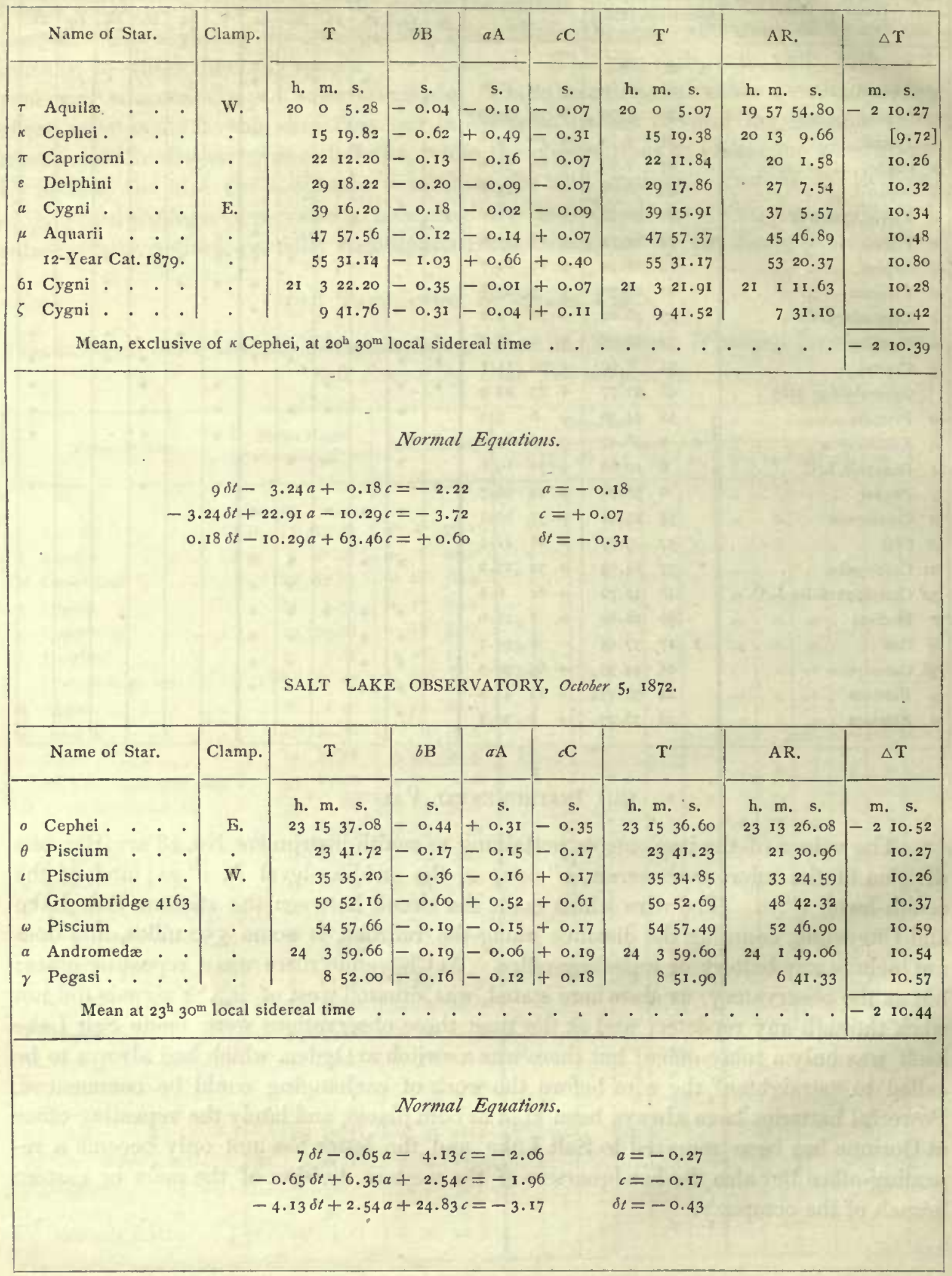


(9.) Uniform Tables of Time-Reductions at Receiving-Station-Continued.

SALT LAKE OBSERVATORY, October 14, 1872.

\begin{tabular}{|c|c|c|c|c|c|c|c|c|c|c|c|c|c|c|c|c|}
\hline & Name of Star & & Clamp. & & $\mathrm{T}$ & & $b \mathrm{~B}$ & & $a \mathrm{~A}$ & & ${ } \mathrm{C}$ & & $\mathbf{T}^{\prime}$ & & AR. & $\Delta \mathbf{T}$ \\
\hline$\tau$ & Aquila. & . & E. & $\begin{array}{l}\mathrm{h} . \\
20\end{array}$ & $\begin{array}{l}\text { m. s. } \\
\text { o I } 7.86\end{array}$ & - & $\begin{array}{l}\text { s. } \\
0.08\end{array}$ & - & $\begin{array}{c}\text { s. } \\
0.14\end{array}$ & & $\begin{array}{l}\text { s. } \\
0.10\end{array}$ & $\begin{array}{l}\mathrm{h} . \\
20\end{array}$ & $\mathrm{~m}$ & $\begin{array}{c}s . \\
17 \cdot 54\end{array}$ & $\begin{array}{l}\text { h. m. s. } \\
\text { I9 } 57 \quad 54.66\end{array}$ & $\begin{array}{c}\text { m. } \quad \text { s. } \\
-222.88\end{array}$ \\
\hline$\theta$ & Aquila. &. & - & t & 76.86 & - & 0.06 & - & 0.17 & - & 0.10 & & 7 & 6.53 & $20 \quad 4 \quad 43.55$ & 22.98 \\
\hline$\kappa$ & Cephei . & .. & - & 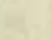 & 1531.66 & - & 0.25 & + & 0.68 & - & 0.46 & - & I5 & 31.63 & 138.71 & 22.92 \\
\hline$\pi$ & Capricorni. & . $\cdot$ & - & 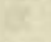 & 2224.56 & - & 0.03 & - & 0.23 & - & O. I I & t & 22 & $24 \cdot 19$ & $20 \quad 1.44$ & 22.75 \\
\hline$\varepsilon$ & Delphini · & - $\quad$ & - & tis & 2930.56 & - & 0.05 & - & 0.13 & - & 0.10 & 4 & 29 & 30.23 & 277.40 & 22.88 \\
\hline & Groombridge & $324 I$ & - & 1 & $3255.7^{8}$ & - & 0.16 & + & 0.42 & - & 0.33 & & 32 & $55.7 \mathrm{I}$ & 3032.87 & 22.84 \\
\hline$a$ & Cygni . . & $\cdot \quad \cdot$ & W. & & 3928.28 & - & 0.09 & + & 0.02 & + & 0.14 & 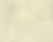 & 39 & 28.35 & $37 \quad 5 \cdot 34$ & 23.00 \\
\hline$\mu$ & Aquarii . & .. & - & & $4^{8} \quad 9.96$ & - & 0.04 & - & 0.20 & + & 0.10 & & $4^{8}$ & 9.82 & 4546.76 & 23.06 \\
\hline & 12-Year Cat. & I 879. & - & 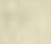 & 5540.70 & - & 0.13 & + & $0.9^{2}$ & + & 0.58 & 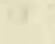 & 55 & 42.07 & $53 \quad 19.25$ & 22.82 \\
\hline $6 I$ & Cygni . & - & - & $2 \mathrm{I}$ & $334 \cdot 30$ & + & 0.01 & - & 0.02 & + & 0.13 & 21 & 3 & $34 \cdot 42$ & $21 \quad I \quad 11,45$ & 22.97 \\
\hline$\zeta$ & Cygni . & . . & - & & 954.05 & - & 0.03 & 1- & 0.06 & $1+$ & 0.12 & 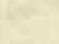 & 9 & 54.08 & 730.95 & 23.13 \\
\hline & Mean at & $20^{\mathrm{h}} 30^{1}$ & ocals & & time & . & & , & - & & - & - & - & & $\cdot \cdot \cdot \cdot$ & -222.93 \\
\hline
\end{tabular}

Normal Equations.

$\begin{array}{rr}1 \mathrm{I} \delta t-4.46 a+1.25 c=+1.73 & a=-0.250 \\ -4.46 \delta t+26.19 a+5.21 c=-7.89 & c=-0.096 \\ 1.25 \delta t+5.21 a+75.02 c=-8.35 & \delta t=+0.070\end{array}$

SALT LAKE OBSERVATORY, October $14,1872$.

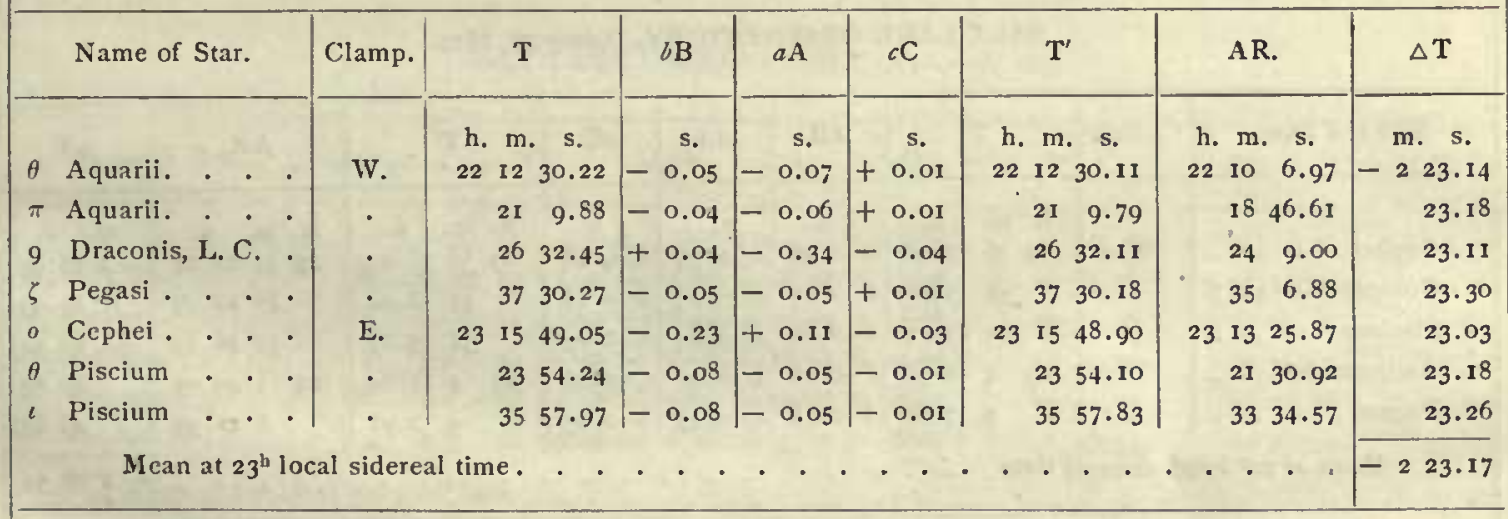

Normal Equations.

$$
\begin{array}{rr}
7 \delta t+5.68 a-5.79 c=-1.78 & a=-0.09 \\
5.68 \delta t+17.39 a-11.98 c=-2.72 & c=+0.01 \\
-5.79 \delta t-11.98 a+29.56 c=+1.70 & \delta t=-0.18
\end{array}
$$


(9.) Uniform Tables of Tmme-Reductions at Receiving-Station-Continued.

SALT LAKE OBSERVATORY, October I6, 1872.

\begin{tabular}{|c|c|c|c|c|c|c|c|c|c|c|c|c|c|c|c|}
\hline & Name of Star. & Clamp. & & $\mathrm{T}$ & s & & $6 \mathrm{~B}$ & $a \mathrm{~A}$ & $c \mathrm{C}$ & & $T^{\prime}$ & & & AR. & $\Delta \mathrm{T}$ \\
\hline$\pi$ & Capricorni & W. & $\begin{array}{l}\text { h. } \\
20\end{array}$ & $\begin{array}{l}\mathrm{m} . \\
22\end{array}$ & $\begin{array}{c}\text { s. } \\
27.72\end{array}$ & & $\begin{array}{c}\text { s. } \\
0.04\end{array}$ & $\begin{array}{c}\text { s. } \\
+\quad 0.16\end{array}$ & $\begin{array}{c}\text { s. } \\
. \quad \text {. }\end{array}$ & $\begin{array}{l}\text { h. } \\
20\end{array}$ & m. & $\begin{array}{c}\text { s. } \\
27.84\end{array}$ & $\begin{array}{l}\text { h. } \\
20\end{array}$ & $\begin{array}{ll}\text { m. } & \text { s. } \\
20 & \text { I. } 41\end{array}$ & $\begin{array}{c}\text { m. s. } \\
-226.43\end{array}$ \\
\hline$\varepsilon$ & Delphini . . & - & 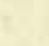 & 29 & $33.6 \mathrm{I}$ & - & 0.09 & +0.09 & .. & & 293 & $33.6 \mathrm{I}$ & & $27 \quad 7.37$ & 26.24 \\
\hline & Groombridge $324 \mathrm{I}$ & . & t & 32 & 59.45 & - & 0.28 & -0.30 & & & 325 & 58.87 & & 3032.72 & 26.15 \\
\hline$a$ & Cygni . . . & . & & 39 & 31.84 & - & 0.25 & -0.02 & . & & 393 & 31.57 & & $37 \quad 5.29$ & 26.28 \\
\hline$\mu$ & Aquarii. . . & . & 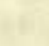 & 48 & 13.16 & - & 0.13 & +0.14 & . & & 48 & 13.17 & & $45 \quad 46.74$ & 26.43 \\
\hline & 12-Year Cat., I 879 & E. & & 55 & 47.17 & - & 0.99 & -0.66 & . & & 55 & $45 \cdot 52$ & & $53 \times 8.99$ & 26.53 \\
\hline $6 \mathrm{r}$ & Cygni . . . & - & $2 \mathrm{I}$ & 3 & $37 \cdot 7^{8}$ & - & 0.09 & +0.01 & - & $2 \mathrm{I}$ & 33 & $37 \cdot 70$ & $2 I$ & 1 II.4I & 26.2 \\
\hline$\zeta$ & Cygni . . & - & & 9 & 57.05 & - & 0.08 & +0.04 & - & 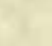 & 95 & 57.01 & & 730.92 & 26.06 \\
\hline$a$ & Cephei. & - & & I7 & 59.12 & + & 0.02 & $-0.1_{4}$ & . & & 17 & 59.00 & & 1532.72 & 26.2 \\
\hline$\beta$ & Aquarii. . & - & & 27 & 17.73 & + & 0.01 & +0.13 & - & & 27 & 17.87 & & 2451.19 & 26. \\
\hline$\beta$ & Cephei . . & . & & 29 & $27 \cdot 95$ & + & 0.03 & -0.26 & . & & 292 & 27.72 & & $27 \quad 1.34$ & 26.3 \\
\hline$\xi$ & Aquarii. . & - & & 33 & 24.70 & + & $0.0 \mathrm{I}$ & +0.13 & - & & 33 & 24.84 & & $305^{8.26}$ & 26.5 \\
\hline$\varepsilon$ & Pegasi . . & . & & 40 & $22 \cdot 32$ & t+ & 0.01 & +0.09 & - & & 40 & 22.42 & & 375592 & 26.50 \\
\hline & Mean at $2 \mathrm{I}^{\mathrm{b}}$ & & & & & & & $\cdot \cdot$ & & 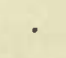 & · & & - & $\cdot \cdot \cdot$ & \\
\hline
\end{tabular}

\section{Normal Equations.}

$$
\begin{array}{rr}
13 \delta t+3.15 a+3.05 c=-5.43 & a=-0.18 \\
3.15 \delta t+20.33 a+21.20 c=-4.81 & c=0.00 \\
3.05 \delta t+21.20 a+68.43 c=-4.33 & \delta t=-0.37
\end{array}
$$

SALT LAKE OBSERVATORY, October $16,1872$.

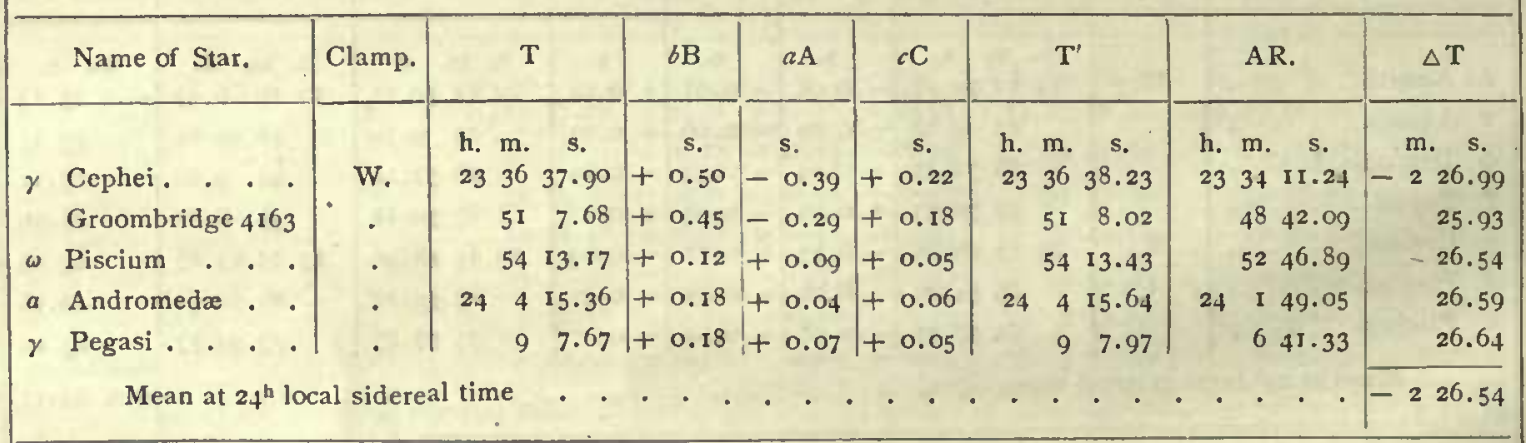

\section{Normal Equations.}

$$
\begin{array}{rlrl}
5 \delta t+3.26 a & =-3.17 & a & =-0.15 \\
3.26 \delta t+11.07 a & =-3.36 & \delta t & =-0.54
\end{array}
$$


(9.) Uniform Tables of Time-Reductions at Receiving-Station-Continued.

SALT LAKE OBSERVATORY, October 18, 1872.

\begin{tabular}{|c|c|c|c|c|c|c|c|c|c|c|c|c|c|c|c|c|}
\hline & Name of Star. & Clamp. & & $\mathbf{T}$ & & & $b \mathbf{B}$ & & $a \mathrm{~A}$ & $c \mathrm{C}$ & & $\mathbf{T}^{\prime}$ & & & AR. & $\Delta T$ \\
\hline 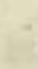 & Groombridge 3241 & W. & $\begin{array}{l}\text { h. } \\
20\end{array}$ & $\begin{array}{l}\mathrm{m} . \\
33\end{array}$ & $\begin{array}{l}\text { s. } \\
3 \cdot 3 I\end{array}$ & - & $\begin{array}{c}\text { s. } \\
0.31\end{array}$ & & $\begin{array}{c}\text { s. } \\
0.08\end{array}$ & $\begin{array}{c}\text { s. } \\
-\quad 0.39\end{array}$ & $\begin{array}{l}\text { h. } \\
20\end{array}$ & $\begin{array}{l}\mathrm{m} . \\
33\end{array}$ & $\begin{array}{l}\text { s. } \\
2.69\end{array}$ & $\begin{array}{l}\text { h. } \\
20\end{array}$ & $\begin{array}{c}\text { m. s. } \\
30 \quad 32.5^{8}\end{array}$ & $\begin{array}{cc}\text { m. s. } \\
-2 & \text { 30. I I }\end{array}$ \\
\hline$a$ & Cygni . . . . & . & & 39 & 35.44 & - & 0.15 & $\therefore$ & 0.01 & -0.17 & & 39 & 35.13 & & $37 \quad 5.23$ & $29 \cdot 90$ \\
\hline$\mu$ & Aquarii. . . & - & 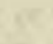 & $4^{8}$ & 17.04 & - & 0.06 & - & 0.04 & -0.12 & & $4^{8}$ & 16.82 & & $45 \quad 46.70$ & 30. I2 \\
\hline 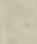 & 12-Year Cat., I 879 & - & & 55. & $49 \cdot 47$ & - & 0.36 & + & 0.18 & -0.69 & & 55 & 48.60 & & $53 \quad 18.73$ & 29.87 \\
\hline $6 I$ & Cygni . & - & $2 \mathrm{I}$ & 3 & $41.4^{8}$ & 一 & 0.09 & & 0.00 & -0.15 & $2 I$ & 3 & 41.24 & 21 & I II.37 & 29.87 \\
\hline$\zeta$ & Cygni . & E. & - & 10 & 0.63 & - & 0.08 & - & 0.01 & +0.14 & & Io & 0.68 & & 730.88 & 29.80 \\
\hline$a$ & Cephei . . & . & & IS & 2.16 & - & 0.07 & + & 0.04 & +0.25 & & I 8 & $2 \cdot 3^{8}$ & & I5 32.64 & $29 \cdot 74$ \\
\hline$\beta$ & Aquarii. & - & & 27 & 20.95 & - & 0.03 & - & 0.04 & +0.12 & & 27 & 21.00 & & 2451.17 & 29.83 \\
\hline$\xi$ & Aquarii. & - & & 33 & 28.01 & $\mp$ & 0.00 & - & 0.04 & +0.12 & & 33 & 28.09 & & 3058.24 & 29.85 \\
\hline$\varepsilon$ & Pegasi . & . & & 40 & $25 \cdot 72$ & $\mp$ & 0.00 & 1- & 0.03 & $1+0.12$ & & 40 & $25.8 \mathrm{I}$ & & 3755.90 & $29.9 \mathrm{I}$ \\
\hline & Mean at $2 \mathrm{I}^{\mathrm{h}}$ lo & 1 sidere & tim & & & . & & & & - & - & - & & & $\cdot$ & -229.90 \\
\hline
\end{tabular}

Normal Equations.

$\begin{array}{rlrl}10 \delta t+3.14 a-6.43 c & =+0.48 & a & =+0.05 \\ 3.14 \delta t+18.99 a-26.71 c & =-\mathrm{I} .8 \mathrm{I} & c & =+0.12 \\ -6.43 \delta t-26.71 a+59.95 c & =+5.86 & \delta t & =+0 . \mathrm{II}\end{array}$

SALT LAKE OBSERVATORY, October I8, 1872.

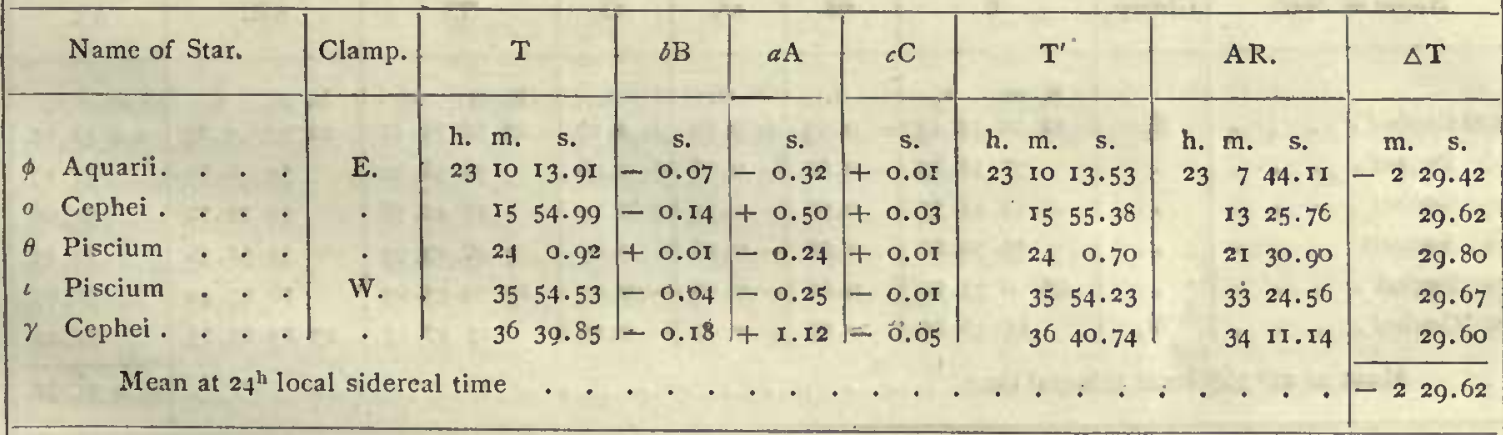

\section{Normal Equations.}

$$
\begin{array}{rl}
5 \delta t-1.85 a-0.79 c=+2.69 & a=-0.43 \\
-1.85 \delta t+9.36 a+9.19 c=-4.66 & c=+0.013 \\
-0.78 d t+9.19 a+29.27 c=-3.93 & \delta t+0.38
\end{array}
$$


(g.) Uniform Tables of Time-Reductions at Receiving-Station-Continued.

SALT LAKE OBSERVATORY, October $19,1872$.

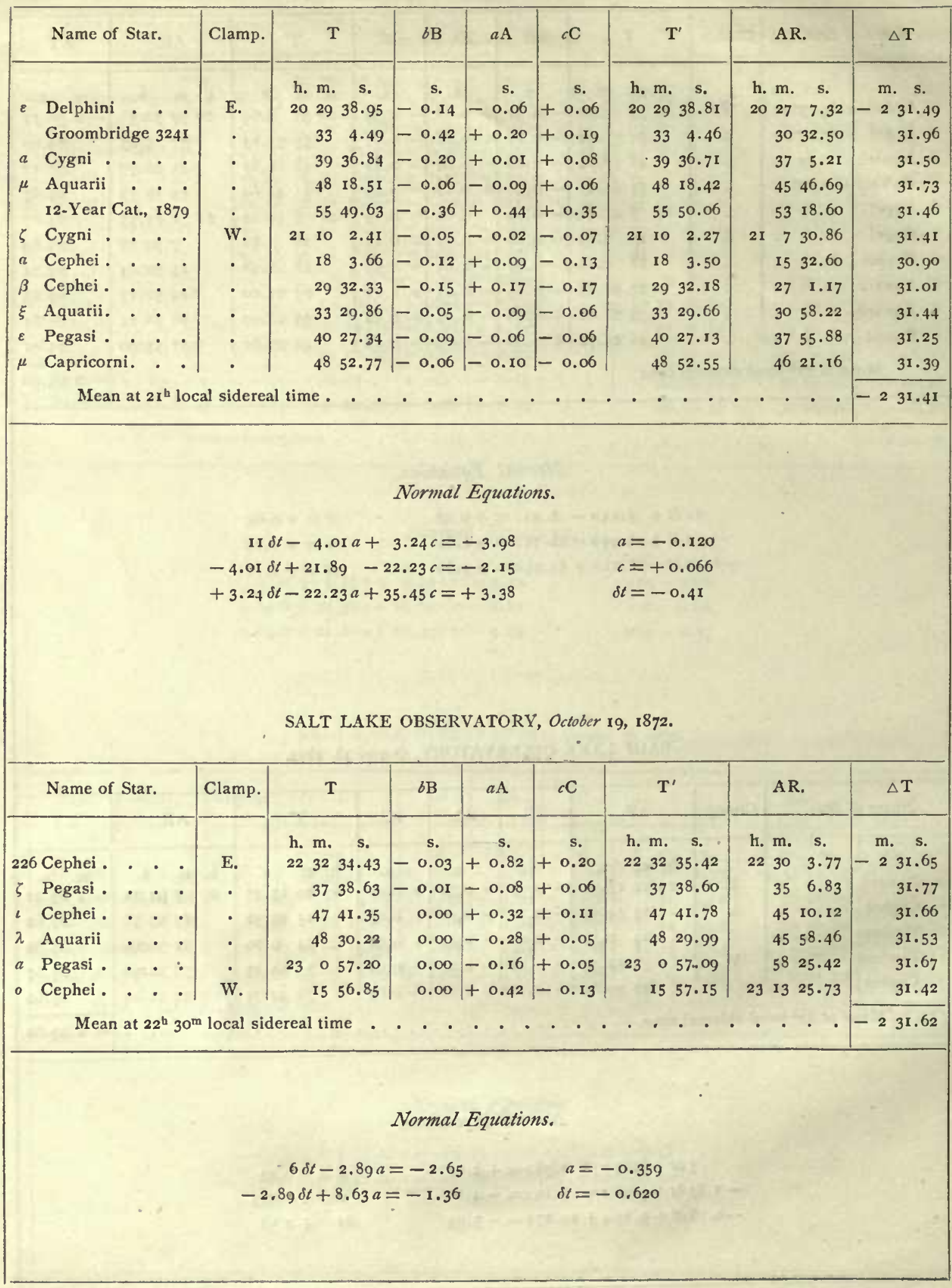


(9.) Uniform Tables of Trme-Reductions at Receiving-Station-Continued.

SALT LAKE OBSERVATORY, October $2 \mathrm{r}, 1872$.

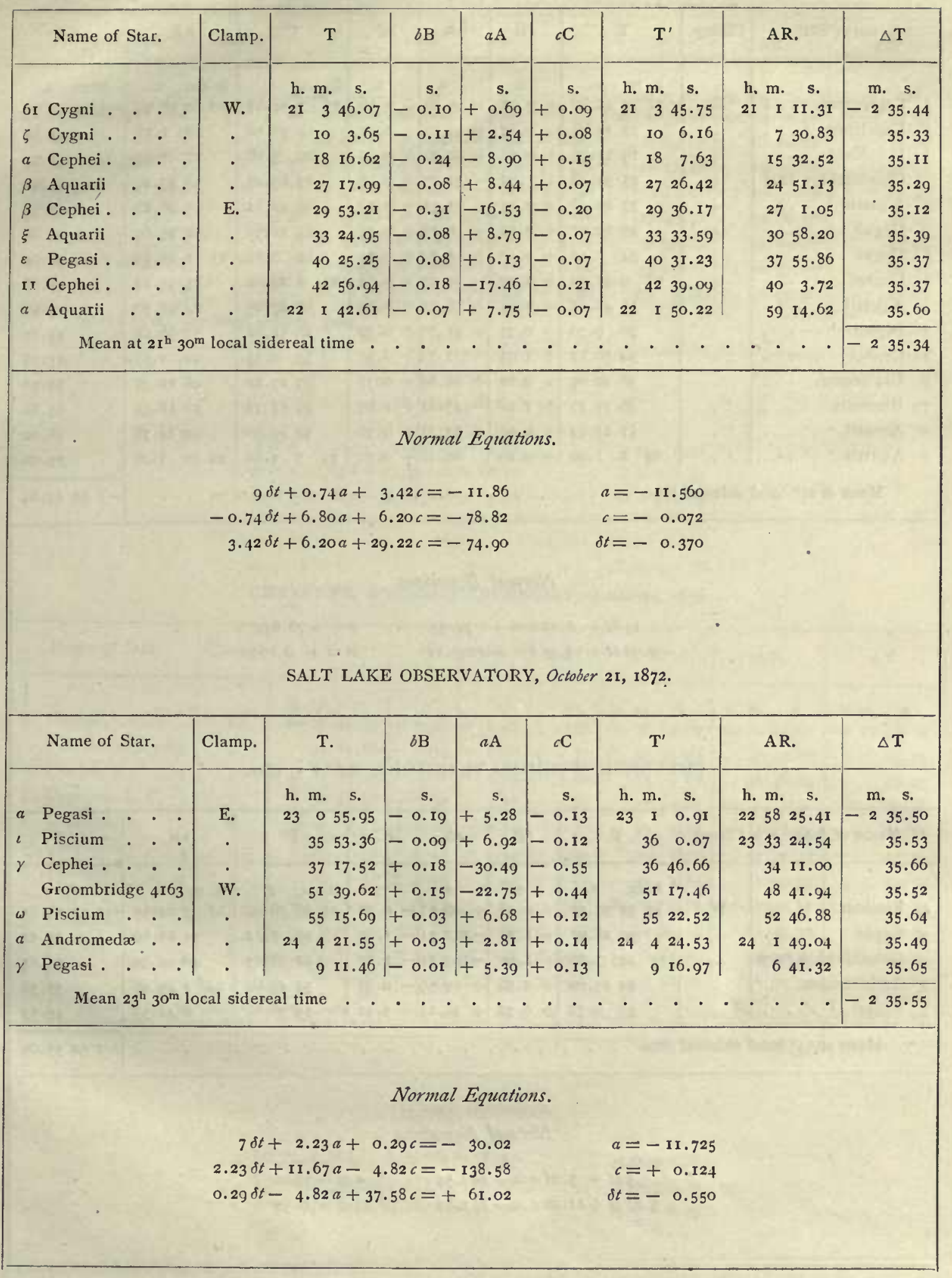


(io.) Uniform Tables of The-Reductions at Sending-Station.

CHEYENNE, WYOMING TERRITORY, October 5, 1872 .

\begin{tabular}{|c|c|c|c|c|c|c|c|c|c|c|}
\hline & Name of Star. & Clamp. & T & T & $6 \mathrm{~B}$ & $a . A$ & $c \mathrm{C}$ & $\mathbf{T}^{\prime}$ & AR. & $\Delta \mathbf{T}$ \\
\hline$a^{2}$ & corni. . & E. & $\begin{array}{l}\text { h. } \mathrm{m} \text {. } \\
222\end{array}$ & $\begin{array}{c}\text { s. } \\
48.80\end{array}$ & $\begin{array}{c}\text { s. } \\
+0.22\end{array}$ & $\begin{array}{r}\text { s. } \\
+65.35\end{array}$ & $\begin{array}{c}5 . \\
+0.30\end{array}$ & $\begin{array}{ccc}\text { h, m. } & \text { s. } \\
22 & 3 & 54.67\end{array}$ & $\begin{array}{l}\text { h. m. } \\
20 \text { 10 } 5^{8.95}\end{array}$ & $\begin{array}{l}\text { h. m. s. } \\
- \text { I } 5255.7\end{array}$ \\
\hline$\pi$ & Capricorni. . . & . & II & 144.94 & +0.19 & +71.72 & +0.31 & 1257.16 & $\begin{array}{lr}20 & \text { I. } 58\end{array}$ & 55.5 \\
\hline$\varepsilon$ & Delphini . . & . & I9 & 922.33 & +0.31 & +40.34 & +0.30 & $20 \quad 3.28$ & $27 \quad 7.54$ & 55.7 \\
\hline & Groombridge $324 \mathrm{I}$ & . & 25 & 539.18 & +1.03 & -131.73 & $+0.9^{8}$ & 2329.46 & $30 \quad 33.49$ & $55 \cdot 9$ \\
\hline$\mu$ & Aquarii . . & . & 37 & 740.48 & +0.25 & $+6 \mathrm{I} .5^{8}$ & +0.30 & $3842.6 \mathrm{I}$ & $45 \quad 46.87$ & 55. \\
\hline & Cygni . . . & - & 45 & 520.01 & +0.52 & +0.78 & +0.39 & 4521.70 & 5225.69 & 3.0 \\
\hline $6 \mathrm{r}$ & Cygni . • • & - & & +1.52 & +0.50 & $\vdash \quad 5.27$ & +0.38 & $54 \quad 7.67$ & $21 \quad I \quad 11.63$ & 6.0 \\
\hline$a$ & Cephei . . . & . & 239 & $927 \cdot 52$ & +0.79 & -59.93 & +0.64 & $23 \quad 8 \quad 29.02$ & 1533.14 & 5. \\
\hline$\beta$ & Aquarii . . & . & 16 & 648.55 & +0.28 & +58.12 & +0.30 & I7 47.25 & 2451 & 5 . \\
\hline & Pegasi . . & . & & o 9.30 & +0.34 & +41.72 & +0.30 & 3051.66 & 3756.05 & 5 . \\
\hline II & Cephei. . . & . & 34 & 456.12 & +1.05 & $-1 \times 7.73$ & +0.91 & $\begin{array}{ll}33 & 0.35\end{array}$ & $40 \quad 4.57$ & 55.7 \\
\hline & Capricorni. & - & $3^{8}$ & 8 ro.or & +0.22 & +66.69 & $+0.3 I$ & 3917.22 & $462 x \cdot 32$ & $55 \cdot$ \\
\hline 79 & Draconis . . & - & 46 & 635.77 & +1.16 & $-\mathrm{I} 43.2 \mathrm{I}$ & +1.03 & 4414.75 & $51 \quad 18.93$ & \\
\hline$a$ & Aquarii. . . & . & $5 \mathrm{I}$ & I 17.44 & +0.29 & +52.77 & +0.30 & 5210.80 & .5914 .78 & 56 \\
\hline$\theta$ & Aquarii. . . & - & 242 & $2 \quad 1.92$ & $1+0.26$ & 60.48 & $1+0.30$ & $\begin{array}{lll}24 & 3 & 2.96\end{array}$ & $2210 \quad 7.06$ & 55.90 \\
\hline & Mean at $2 \mathrm{I}^{\mathrm{b}}$ lo & & & & & & & & & \\
\hline
\end{tabular}

\section{Normal Equations.}

$\begin{array}{rlrl}\mathrm{I}_{5} \delta t-0.92 a & =+74.33 & a & =-78.642 \\ -0.92 \delta t+13.95 a & =-1097.1 \mathrm{I} & \delta t & =+0.160\end{array}$

CHEYENNE, WYOMING TERRITORY, October 5, 1872.

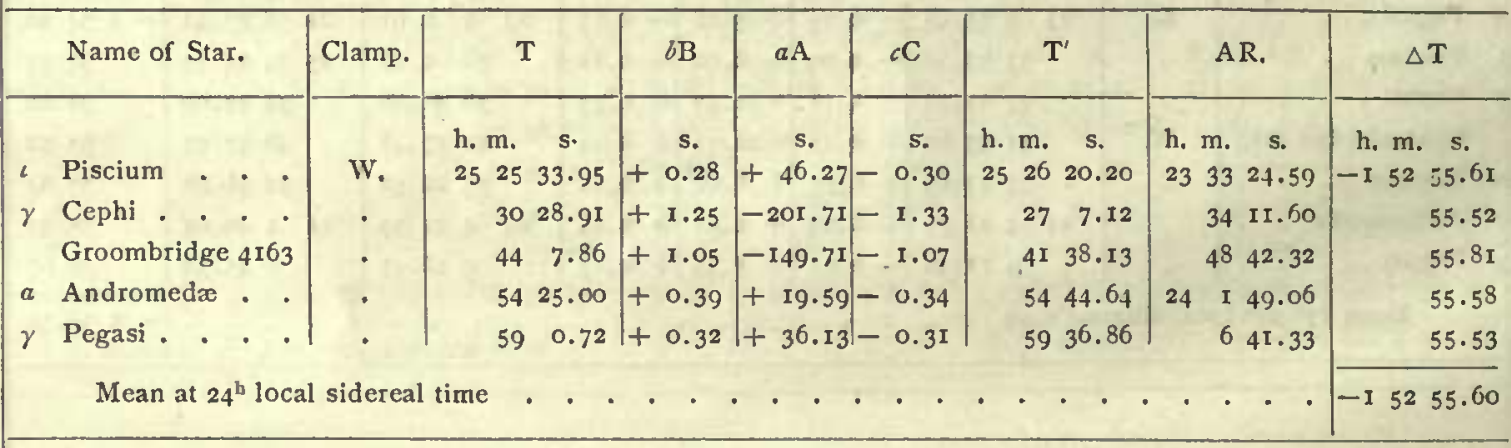

\section{Normal Equations.}

$$
\begin{array}{rlrl}
58 t+3.21 a & =-247.54 & a & =-78.00 \\
3.21 \delta t+11.02 a & =-857.87 & \delta t & =+0.58
\end{array}
$$


(io.) Uniform Tables of Time-Reductions at Receiving-Station-Continued. CHEYENNE, WYOMING TERRITORY, October I4, I872.

\begin{tabular}{|c|c|c|c|c|c|c|c|c|c|c|c|}
\hline & Name of Star. & Clamp. & & $\mathbf{T}$ & $6 \mathrm{~B}^{\circ}$ & $a \mathrm{~A}$ & $\iota \mathrm{C}$ & $\mathrm{T}^{\prime}$ & AR. & & $T$ \\
\hline$\theta$ & Aquarii. . & W. & $\begin{array}{lr}\text { h. } & \text { m. } \\
24 & 2\end{array}$ & $\begin{array}{c}s . \\
50.84\end{array}$ & $\begin{array}{c}s . \\
+0.05\end{array}$ & $\begin{array}{c}\text { s. } \\
-0.70\end{array}$ & $\begin{array}{c}s . \\
-0.35\end{array}$ & $\begin{array}{llc}\text { h. } & \text { m. } & \text { s. } \\
24 & 2 & 49.84\end{array}$ & $\begin{array}{llc}\text { h. } & \text { m. } & \text { s. } \\
22 & \text { 10 } & 6.97\end{array}$ & $\begin{array}{r}\text { 1. m. } \\
-\mathrm{I} 52\end{array}$ & $\begin{array}{c}\text { s. } \\
42.87\end{array}$ \\
\hline & Aquarii. & . & IO & $30.5 \mathrm{I}$ & +0.06 & -0.59 & -0.35 & Io 29.63 & I8 46.6 I & & 43.02 \\
\hline 9 & Draconis, L. C. & . & 16 & 54.06 & -0.16 & -3.42 & +1.48 & $16 \quad 51.96$ & 249.01 & & 42.95 \\
\hline 226 & 5 Cephei. . & . & 22 & 46.08 & +0.26 & +2.07 & -1.40 & 2247.01 & $30 \quad 4.06$ & & 42.95 \\
\hline$\zeta$ & Pegasi . ' & . & 27 & 50.73 & +0.07 & $-0.4^{8}$ & $-0.3^{6}$ & 2749.96 & $35 \quad 6.88$ & & 43.08 \\
\hline$\iota$ & Cephei. . . & . & 38 & 53.05 & +0.17 & +0.91 & -0.84 & $38 \quad 53.29$ & .4510 .27 & & 43.02 \\
\hline$a$ & Piscis Australis & . & 43 & 21.71 & +0.03 & -1.00 & $-0.4 \mathrm{I}$ & 4320.33 & 5037.19 & & 43.14 \\
\hline$a$ & Pegasi . . . & . & 50 & 935 & +0.07 & -0.42 & -0.36 & 50 8.64 & $5^{8} \quad 25 \cdot 4^{6}$ & & 43.18 \\
\hline
\end{tabular}

Normal Equations.

\begin{tabular}{|c|c|}
\hline $8 \delta t+3.99 a-7.40 c=-6.38$ & $a=-0.9 \mathrm{x}$ \\
\hline $3.99 \delta t+22.89 a+23.74 c=-12.73$ & $c=+0.35$ \\
\hline $7.408 t+23.74 a+45.34 c=-5.45$ & $\delta t=-0.02$ \\
\hline
\end{tabular}

CHEYENNE, WYOMING TERRITORY, October I4, 1872.

\begin{tabular}{|c|c|c|c|c|c|c|c|c|c|c|c|c|c|c|c|c|c|c|c|c|}
\hline \multicolumn{5}{|c|}{ Name of Star. } & & \multicolumn{2}{|r|}{$\mathbf{T}$} & \multirow{2}{*}{\multicolumn{2}{|c|}{$\begin{array}{c}b \mathrm{~B} \\
\begin{array}{c}\mathrm{s} . \\
+0.46\end{array}\end{array}$}} & \multirow{2}{*}{\multicolumn{2}{|c|}{$\begin{array}{c}a \mathrm{~A} \\
\begin{array}{c}\mathrm{s} . \\
+\quad \mathbf{1 . 9 7}\end{array}\end{array}$}} & \multirow{2}{*}{\multicolumn{2}{|c|}{$\begin{array}{c}c \mathrm{C} \\
s . \\
+0.88\end{array}$}} & \multicolumn{3}{|c|}{$\mathbf{T}^{\prime}$} & \multicolumn{2}{|r|}{ AR. } & \multicolumn{2}{|c|}{$\Delta \mathrm{T}$} \\
\hline$\gamma$ & Cephei . & . & . & & & $\begin{array}{l}\text { h. } \\
25\end{array}$ & ${ }_{26}$ m. $_{51.23}$ & & & & & & & $\begin{array}{l}\text { h. } \\
25\end{array}$ & & $\begin{array}{c}\text { s. } \\
54.54\end{array}$ & & $\begin{array}{lc}\text { m. } & \text { s. } \\
34 & \text { II. } 30\end{array}$ & $\begin{array}{r}\text { h. } m . \\
-I 52\end{array}$ & $\begin{array}{c}\text { s. } \\
43.24\end{array}$ \\
\hline & Groombrid & dge & 4163 & & . & . & 4122.86 & + & 0.39 & + & 1.46 & + & $0.7 \mathrm{I}$ & & $4 \mathrm{I}$ & $25 \cdot 42$ & & $\begin{array}{lll}4^{8} & 42.13\end{array}$ & & 43.29 \\
\hline$\omega$ & Piscium & . & . & & . & & 4530.00 & + & 0.10 & - & 0.44 & + & 0.20 & & 45 & 29.86 & & $\begin{array}{ll}52 & 46.89\end{array}$ & & 42.97 \\
\hline 4 & Draconis, I & L. $C$ & c. & • & - & & $58 \quad 55.60$ & - & 0.32 & - & 3.27 & - & 0.99 & & & 51.02 & 24 & 67.60 & & $43 \cdot 40$ \\
\hline$a$ & Cassiopeiac & & . & & W. & 26 & $26 \quad 1.77$ & + & 0.10 & + & 0.34 & - & 0.36 & 26 & 26 & 1.85 & & 3318.71 & & 43.14 \\
\hline 21 & Cassiopeix & & - & & - & & $30 \quad 1.80$ & + & 0.18 & + & 1.54 & - & 0.75 & & 30 & 2.77 & & 3719.31 & & $43 \cdot 46$ \\
\hline$\varepsilon$ & Piscium & . & - & • & - & & $49 \quad 4 \cdot 42$ & + & 0.05 & - & 0.43 & - & 0.20 & & 49 & 3.84 & & 5620.74 & & 43.10 \\
\hline$\theta^{\mathrm{l}}$ & Ceti . & . & - & • & - & 27 & I0 24.33 & + & 0.04 & - & 0.59 & - & 0.20 & 27 & 10 & $23.5^{8}$ & 251 & I7 40.21 & & $43 \cdot 37$ \\
\hline 38 & Cassiopeix & $\infty$ & . & & - & & I4 31.84 & + & 0.15 & + & 1.04 & - & 0.57 & & & 32.46 & & 2149.26 & & 43.20 \\
\hline$\eta$ & Piscium & . & . & & . & & I7 $24.5^{8}$ & + & 0.07 & - & 0.35 & - & 0.21 & & 17 & 24.09 & & 2440.83 & & 43.26 \\
\hline 0 & Piscium & . & . & • & . & & $31 \quad 24.62$ & + & 0.05 & - & $0.4 \mathrm{I}$ & - & 0.20 & & $3 \mathrm{I}$ & 24.06 & & $38 \quad 40.93$ & & 43.13 \\
\hline & Mean & at 2 & 24.3 & & local s & & al time & & - . & & & - & & - & & & & . & -152 & 43.23 \\
\hline
\end{tabular}

Normal Equations.

$\begin{array}{rr}11 \delta t-1.12 a-8.35 c=-3.39 & a=-0.76 \\ -1.12 \delta t+36.82 a-29.15 c=-33.68 & c=+0.20 \\ -8.35 \delta t-29.15 a+86.78 c=+42.90 & \delta t=-0.23\end{array}$


(io.) Uniform Tables of Time-Reductions at Receiving-Station-Continued. CHEYENNE, WYOMING TERRITORY, October I6, I872.

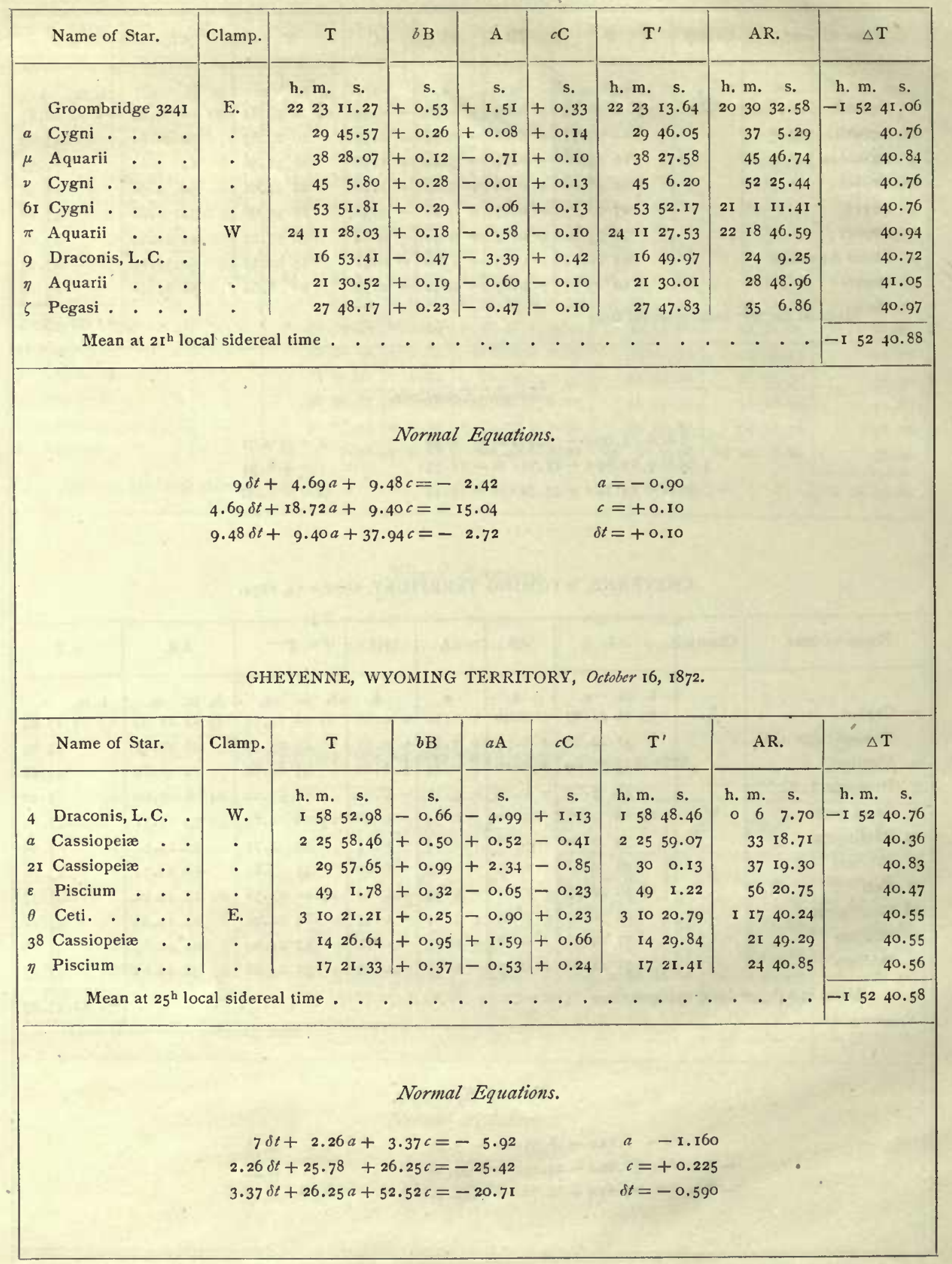


(io.) Uniform Tables of Time-Reductions at Receiving-Station-Continued. CHEYENNE, WYOMING TERRITORY, October $18,1872$.

\begin{tabular}{|c|c|c|c|c|c|c|c|c|c|c|c|c|}
\hline & Name of Star. & Clamp. & & $\mathrm{T}$ & & $6 \mathrm{~B}$ & & $a \mathrm{~A}$ & $c \mathrm{C}$ & $\mathbf{T}^{\prime}$ & AR. & $\Delta \mathrm{T}$ \\
\hline$\sigma^{8}$ & Ursæe Maj., L.C. & W. & $\begin{array}{l}\text { h. } \\
22\end{array}$ & $\begin{array}{l}\text { m. s. } \\
5 \mathrm{I} \quad 48.50\end{array}$ & & $\begin{array}{c}\text { s. } \\
0,14\end{array}$ & - & $\begin{array}{c}\text { s. } \\
3.13\end{array}$ & $\begin{array}{c}\text { s. } \\
+0.52\end{array}$ & $\begin{array}{l}\text { h. m. s. } \\
222 \text { 51 } 45 \cdot 75\end{array}$ & $\begin{array}{cc}\text { h. m. } & \text { s. } \\
59 & 7.95\end{array}$ & $\begin{array}{ccc}\text { h. m. s. } \\
\text {-I } 52 \quad 37.80\end{array}$ \\
\hline$\zeta$ & Cygni . . & . & 23 & o 9.13 & + & 0.17 & - & 0.29 & -0.23 & $23 \circ 8.78$ & 21730.88 & 37.90 \\
\hline$\alpha$ & Cephei . . & - & & 89.66 & + & 0.32 & + & 0.96 & -0.43 & $810.5 \mathrm{r}$ & I5 32.64 & 37.87 \\
\hline I & Draconis, L. C. & - & & II 25.78 & - & 0.62 & - & $7 \cdot 4^{8}$ & +1.42 & II I9. IO & 9 ז8 4 I .04 & 38.06 \\
\hline$\beta$ & Cephei. . & . & & 1937.72 & + & $0.5 \mathrm{I}$ & + & 1.78 & -0.59 & I9 39.42 & $21 \quad 27 \quad 1.22$ & 38.20 \\
\hline$\xi$ & Aquarii. & - & & $23 \quad 37: 34$ & + & 0.13 & - & 0.97 & -0.20 & $233^{6.30}$ & 3058.24 & 38.06 \\
\hline$\varepsilon$ & Pegasi . & - & & $30 \quad 34.49$ & + & 0.17 & - & 0.67 & -0.20 & $30 \quad 33.79$ & 3755.90 & 37.89 \\
\hline II & Cephei . & . & & 3240.02 & + & 0.53 & + & 1.89 & $-0.6 \mathrm{I}$ & 3241.83 & $40 \quad 3.88$ & $37 \cdot 95$ \\
\hline$\mu$ & Capricorni . & - & & $\begin{array}{ll}39 & 0.4 I\end{array}$ & + & 0.14 & - & 1.07 & $-0.2 I$ & 3859.27 & 4621.17 & 38.10 \\
\hline 79 & Draconis & & & 4354.07 & $1+$ & $0.5^{8}$ & 1+ & 2.29 & -0.69 & $43 \quad 56.25$ & 5118.18 & 38.07 \\
\hline & Mean at $22^{\mathrm{h}}$ & sider & & e. & & & & & 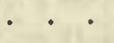 & • & . & $\begin{array}{lll}-1 & 52 & 37.99\end{array}$ \\
\hline
\end{tabular}

\section{Normal Equations.}

\begin{tabular}{|c|c|}
\hline $5.33 \delta t+51.26 a=-64.32$ & $\delta t=+0.010$ \\
\hline
\end{tabular}

CHEYENNE, WYOMING TERRITORY, October $18,1872$.

\begin{tabular}{|c|c|c|c|c|c|c|c|c|c|c|c|c|c|}
\hline & Name of Srar. & Clamp. & $\mathrm{T}$ & & & $6 \mathrm{~B}$ & $a \mathrm{~A}$ & cC & $\mathbf{T}^{\prime}$ & \multicolumn{2}{|r|}{ AR. } & \multicolumn{2}{|c|}{$\Delta \mathbf{T}$} \\
\hline$\iota$ & Piscium & W. & $\begin{array}{l}\text { h. } \mathrm{m} \\
2526\end{array}$ & $\begin{array}{l}\text { s. } \\
2.90\end{array}$ & + & $\begin{array}{c}\text { s. } \\
0.02\end{array}$ & $\begin{array}{c}s . \\
-0.5^{8}\end{array}$ & $\begin{array}{c}s . \\
-0.20\end{array}$ & $\begin{array}{ccc}\text { h. m. } & \text { s. } \\
25 & 26 & 2.14\end{array}$ & h. & $\begin{array}{c}\text { m. s. } \\
3324.56\end{array}$ & $\begin{array}{r}\text { h. } \mathrm{m} . \\
-\mathrm{I} 52\end{array}$ & s. \\
\hline & Groombridge 4163 & . & $4 \mathrm{I}$ & 18.63 & - & 0.03 & +1.87 & -0.71 & $41 \quad 19.76$ & & $48 \quad 42.03$ & & 37.73 \\
\hline$\omega$ & Piscium . . & - & 45 & 25.25 & - & $0.0 \mathrm{r}$ & $-0.5^{6}$ & -0.20 & $45 \quad 24.48$ & & $5^{2} 4^{6.89}$ & & 37.59 \\
\hline 4 & Draconis, L. C. & . & 58 & 48.54 & + & 0.10 & -4.19 & +0.99 & $5^{8} \quad 45.44$ & 24 & $\begin{array}{ll}6 & 7.77\end{array}$ & & 37.67 \\
\hline$a$ & Cassiopez. . & - & 2624 & $56.2 \mathrm{I}$ & 一 & 0.03 & +0.44 & -0.36 & $\begin{array}{lll}26 & 24 & 56.26\end{array}$ & & 3318.70 & & 37.56 \\
\hline$\beta$ & Ceti. . & - & 29 & $5 \mathrm{I} .3 \mathrm{I}$ & - & 0.02 & -0.89 & $-0.2 \mathrm{I}$ & 2950.19 & & $37 \quad 12.55$ & & 37.64 \\
\hline$\varepsilon$ & Piscium & . & 48 & 59.06 & - & 0.02 & -0.55 & $1-0.20$ & $4^{8} \quad 58.29$ & & 5620.76 & & 37.53 \\
\hline & Mean at $24^{\text {h }}$ lo & 1 sidere & ime & . & & 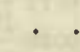 & 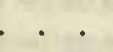 & . & - . & - & . & -152 & 37.61 \\
\hline
\end{tabular}

\section{Normal Equations.}

$$
\begin{aligned}
& 7 \delta t+4.57 a=-1.76 \quad a=-0.975 \\
& 4.57 \delta t+24.19 a=-21.82 \quad \delta t=+0.390
\end{aligned}
$$


(io.) Uniform T'ables of Time-Reductions at Rechiving-Station-Continued. CHEYENNE, WYOMING TERRITORY, Octoher 19,1872 .

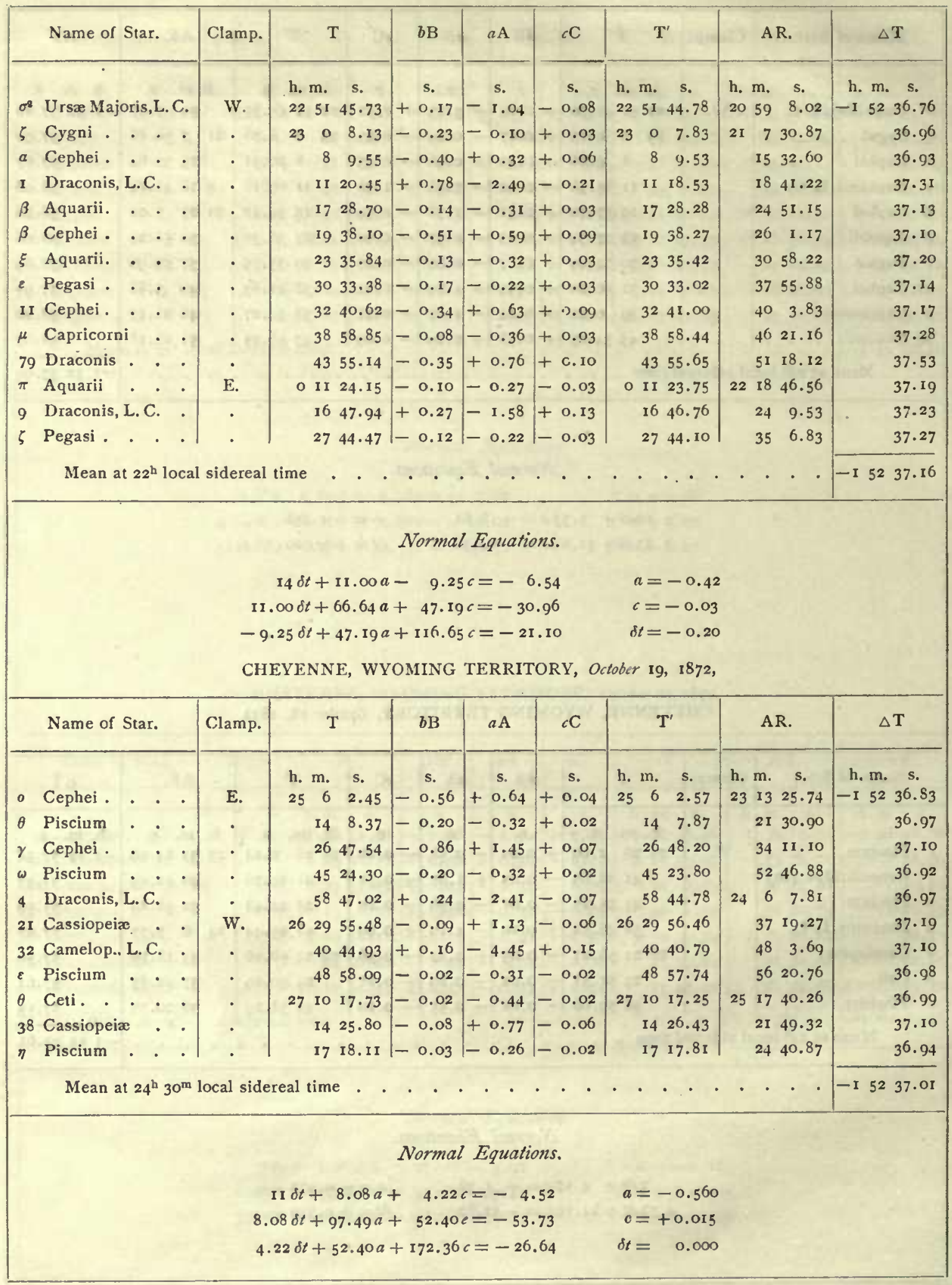


(io.) Uniform Tables of Trme-Reductions at R Receiving-Station-Continued. CHEYENNE, WYOMING TERRITORY, October 21, 1872.

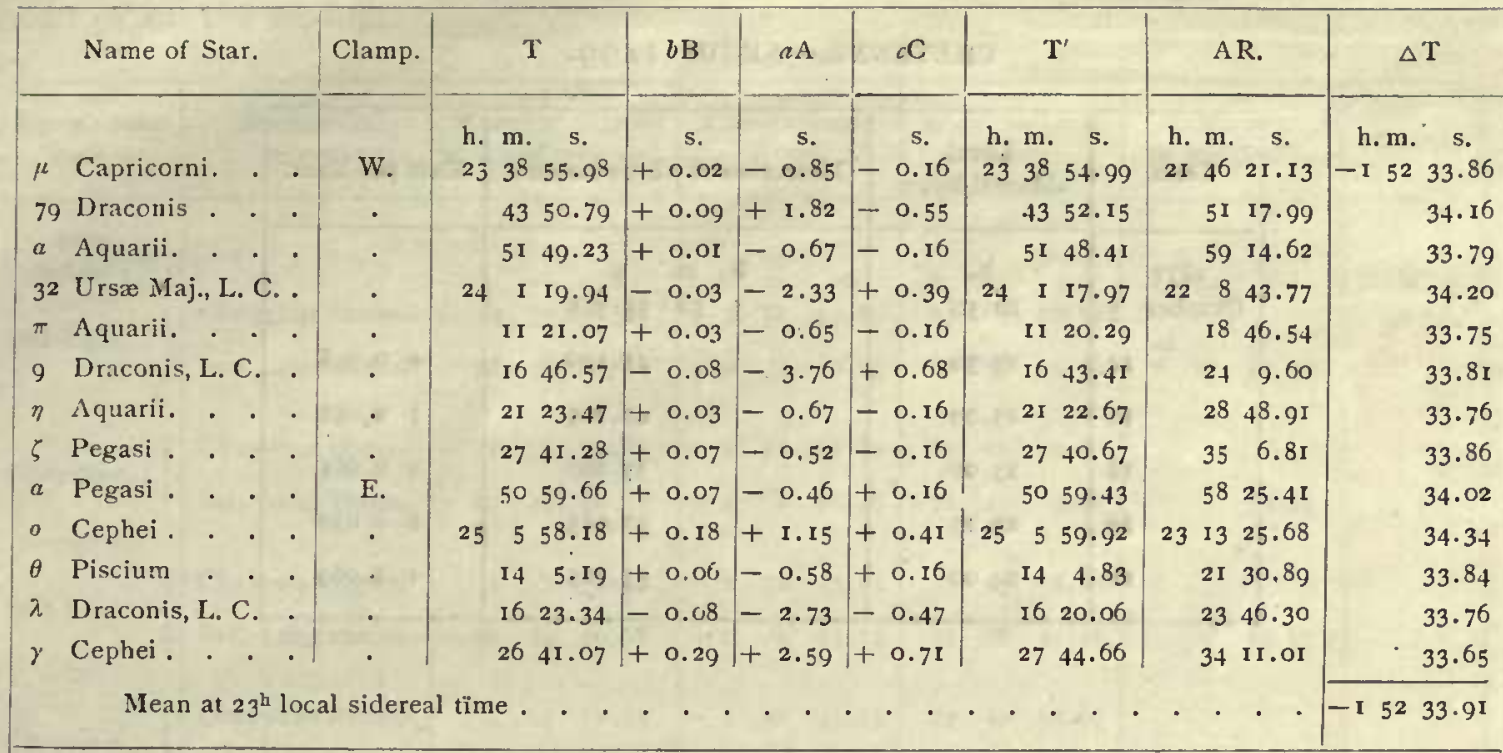

\section{Normal Equations.}

$\begin{array}{rlrl}13 \delta t+7.66 a+4.30 c & =-5.93 & & =-\mathrm{I} .00 \\ 7.66 \delta t+41.22 a+3.07 c & =-39.91 & c & =+0.16 \\ 4.30 \delta t+3.07 a+77.44 c & =+9.96 & \delta t=+0.10\end{array}$

CHEYENNE, WYOMING TERRITNRY, October 21, I872.

\begin{tabular}{|c|c|c|c|c|c|c|c|c|c|c|c|}
\hline & Name of & Star. & lamp. & & $\mathrm{T}$ & $b B$ & $a \mathrm{~A}$ & ${ }^{2} \mathrm{C}$ & $\mathrm{T}^{\prime}$ & AR. & $\Delta T$ \\
\hline$a$ & Cassiopex & - & E. & & $\begin{array}{l}\text { m. S. } \\
2551.28\end{array}$ & $\begin{array}{c}5 . \\
+0.09\end{array}$ & $\begin{array}{c}\text { s. } \\
+0.43\end{array}$ & $\begin{array}{c}5 . \\
+0.25\end{array}$ & 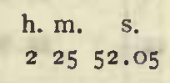 & $\begin{array}{l}\text { h. m. s. } \\
\circ 33 \times 8.69\end{array}$ & 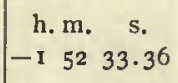 \\
\hline 2 I & Cassiopex & e. . & - & & 2950.46 & +0.15 & +1.92 & +0.52 & 2953.05 & $37 \quad 19.22$ & 33.83 \\
\hline 32 & Camelopa & rd., L. C. & . & & $40 \quad 46.90$ & -0.45 & -7.55 & -1.36 & 4037.54 & $48 \quad 3.75$ & 33.79 \\
\hline$\varepsilon$ & Piscium & . . . & · & & $4^{8} 54.62$ & +0.06 & -0.53 & +0.14 & $4^{8} \quad 54.29$ & $56 \quad 20.76$ & 33.53 \\
\hline$\theta$ & Ceti. . & . & W. & 3 & 1014.40 & +0.07 & -0.74 & -0.14 & 31013.59 & I I $7,40.26$ & 33.33 \\
\hline 38 & Cassiopex & . & - & & 1421.72 & +0.30 & +1.30 & -0.40 & 1422.92 & 2 I 49.3 I & $33.6 \mathrm{I}$ \\
\hline$\eta$ & Piscium & . & . & & 1714.62 & $1+0.15$ & -0.44 & -0.14 & 1714.19 & 2440.87 & $33 \cdot 32$ \\
\hline & Mean & at $25^{1 \mathrm{l}} \mathrm{lc}$ & side & & . & & & . & & 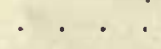 & $\begin{array}{lll}\text { I } & 52 & 33.54\end{array}$ \\
\hline
\end{tabular}

\section{Normal Equations.}

$\begin{array}{rlrl}7 \delta t+5.91 a-8.16 c & =-3.27 & a & =-0.950 \\ 5.91 \delta t+70.49 a-82.36 c & =-75.09 & c & =+0.140 \\ -8.16 \delta t-82.36 a+122.80 c & =+90.95 & \delta t & =+0.490\end{array}$


The following table shows the corrections and the rate of the chronometers used at Cheyenne and Salt Lake City:

CHEYENNE.-NEGUS 1499.

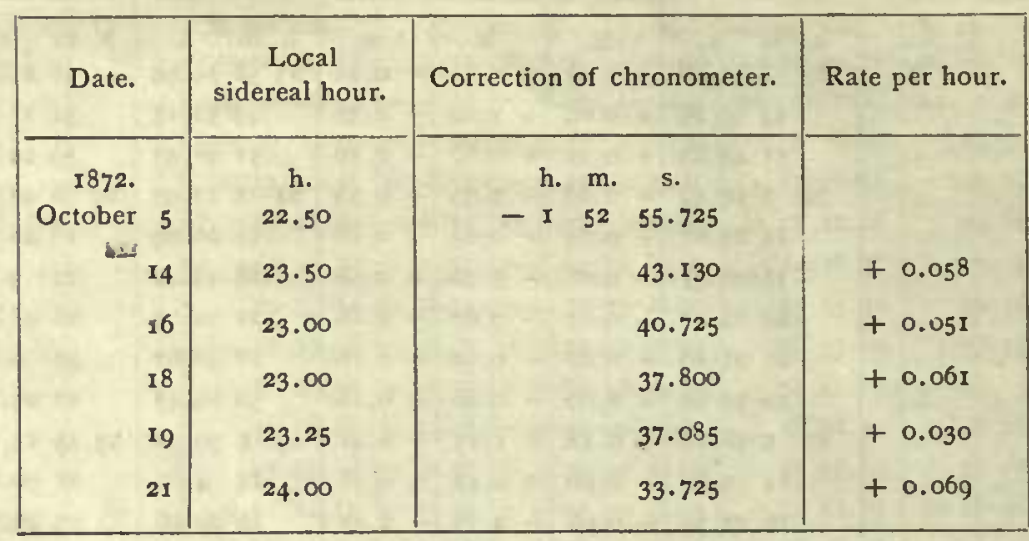

SALT LAKE.-NEGUS I 5 II.

\begin{tabular}{|c|c|c|c|}
\hline Date. & $\begin{array}{l}\text { Local } \\
\text { sidereal hour. }\end{array}$ & Correction of chronometer. & Rate per hour. \\
\hline $\begin{array}{c}1872 . \\
\text { October } 5\end{array}$ & $\begin{array}{c}\text { h. } \\
22.00\end{array}$ & $\begin{array}{ccc}\text { h. m. s. } \\
-02 & 20.415\end{array}$ & \\
\hline 14 & 21.75 & 23.050 & -0.059 \\
\hline 16 & 22.50 & 26.455 & -0.070 \\
\hline 18 & 22.50 & 29.760 & .0 .069 \\
\hline 19 & 21.75 & 31.515 & -0.075 \\
\hline 21 & 22.50 & $\sin 2 x+\frac{1}{10}$ & -0.081 \\
\hline
\end{tabular}




\section{(i i.) Grouping of Series of Exchange-Signals.}

This table shows the result obtained for longitude each way each night and the mean of all the nights:

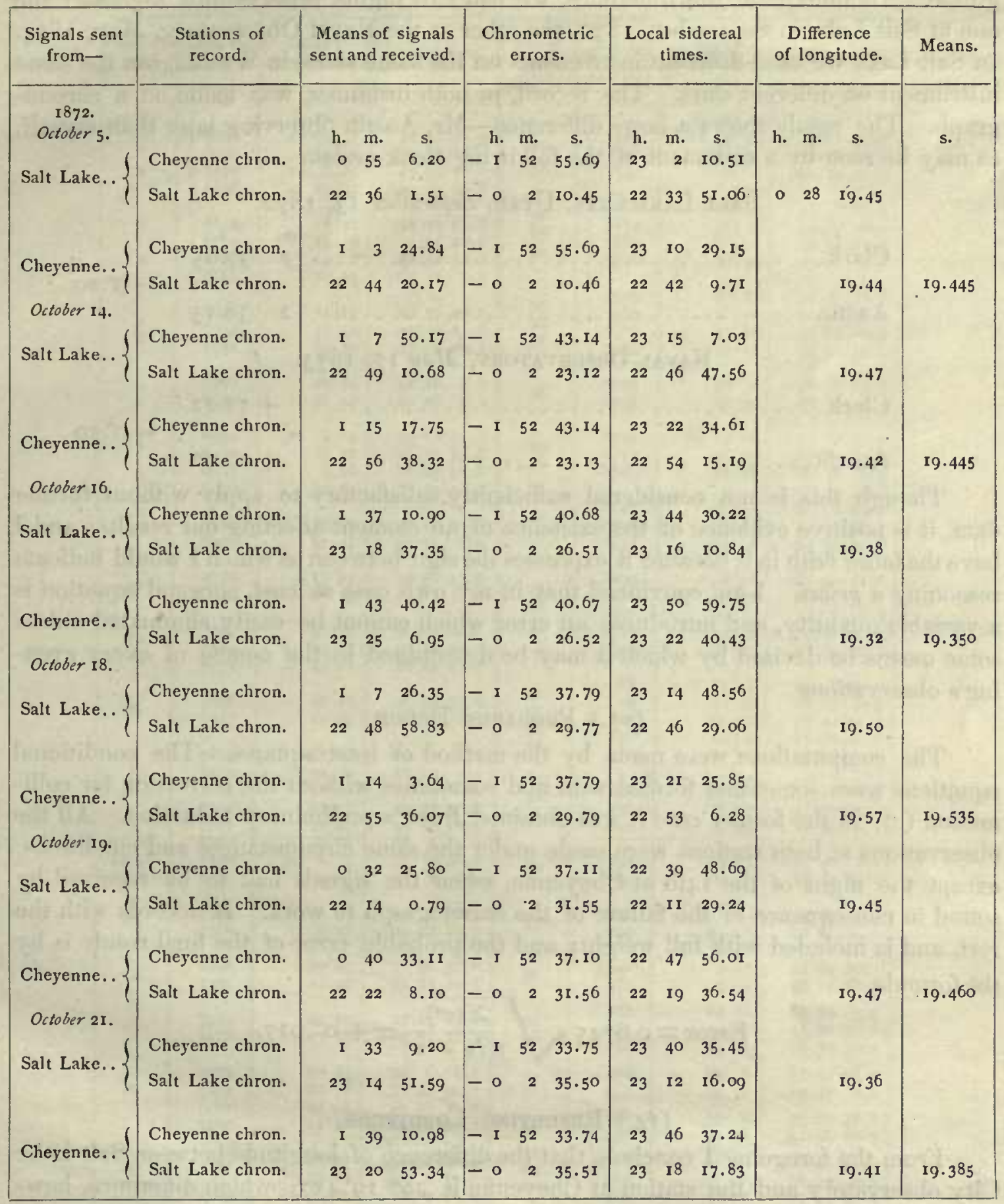

Cheycnne east of Salt Lake City . . . . . $0^{\text {h }} 28^{m} 19^{m} \cdot 437 \pm 0^{8} .017$ 


\section{(i 2.) Personal Equation.}

Whatever differences there may have been between Mr. Austin and myself in recording time, technically known as the personal equation, is still involved in the longitude. To determine this difference, we had two nights' observations together; the one at Salt Lake in September, 1872 , the other at the Naval Observatory, May, i873. In Salt Lake we used different instruments on the same stars, in Washington the same instrument on different stars. The record, in both instances, was made on a chronograph. The result shows a large difference-Mr. Anstin observing later than myself, as may be seen by a statement of the following clock-crrors:

\section{Salt Lake City, Utah, September I3, I872.}

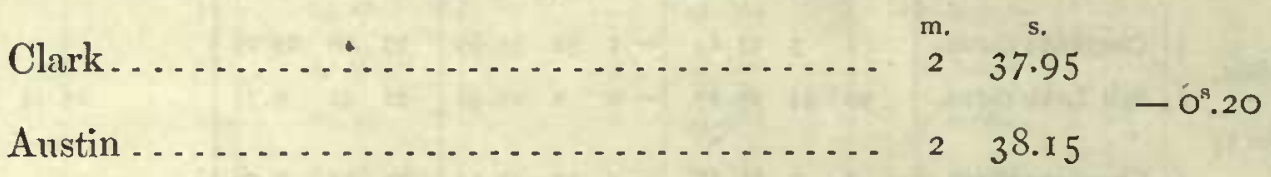

Naval Observatory, May 17, 1873.

Clark . . . . . . . . . . . . . . .

Austin....................... + + +10.92

Though this is not considered sufficiently satisfactory to apply without further data, it is positive evidence of the existence of an element affecting our results; and I have the more faith in it because it expresses the sign between us which I would indicate reasoning a priori. I am convinced that, in my own case at least, personal equation is a variable quantity, and introduces an error which cannot be easily eliminated unless some means be devised by which it may be determined in the course of every evening's observations.

\section{(13.) Probable Error.}

The computations were made by the metlod of least squares. The conditional equations were sometimes formed with and sometimes without the correction for collimation $(c)$; in the former case it was obtained from a preliminary reduction. All the observations at both stations were made under the same circumstances and conditions, except the night of the $14^{\text {th }}$ at Cheyenne, when the signals liad to be received by sound in consequence of the failure of the chronograph to work. It accords with the rest, and is included with full weight; and the probable error of the final result is by the formula,

$$
\text { Error }=0.6745 \wedge \sqrt{\frac{\sum\left(v^{2}\right)}{n(n-1)}}= \pm 0^{\prime \prime} .017
$$

\section{(14.) Resulting Longitude.}

From the foregoing I conclude that the difference of longitude between Salt Lake City observatory and the station at Cheyenne is $28^{\mathrm{m}} 19^{\mathrm{s}} .437$; which difference, however, is still subject to correction for personal equation; and if any weight is to be given to what is indicated as this equation between Mr. Austin and myself, this longitudinal difference will be reduced by the extent of, perhaps, $0^{5} .2$ of a second. 
(15.) Reduction of the Latitude Observations.

Mean Places of Stars for 1872.0 used for Determination of Latitude of Cheyenne, Wyoming Territory.

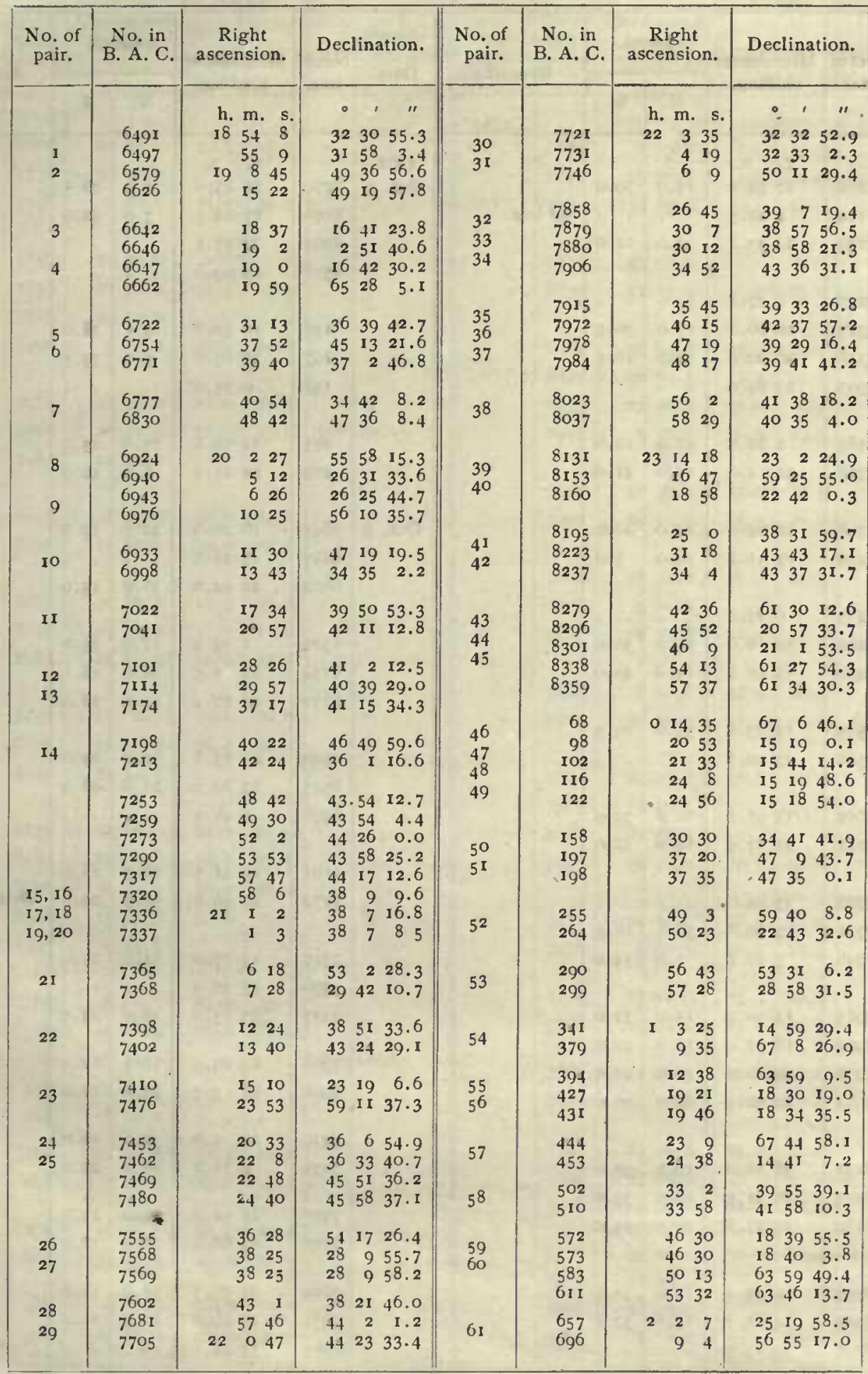


(15.) Reduction of the Latitude-Observations-Continued.

Observations for Latitude.-Station, Cheyenne, Wyoming Territory.

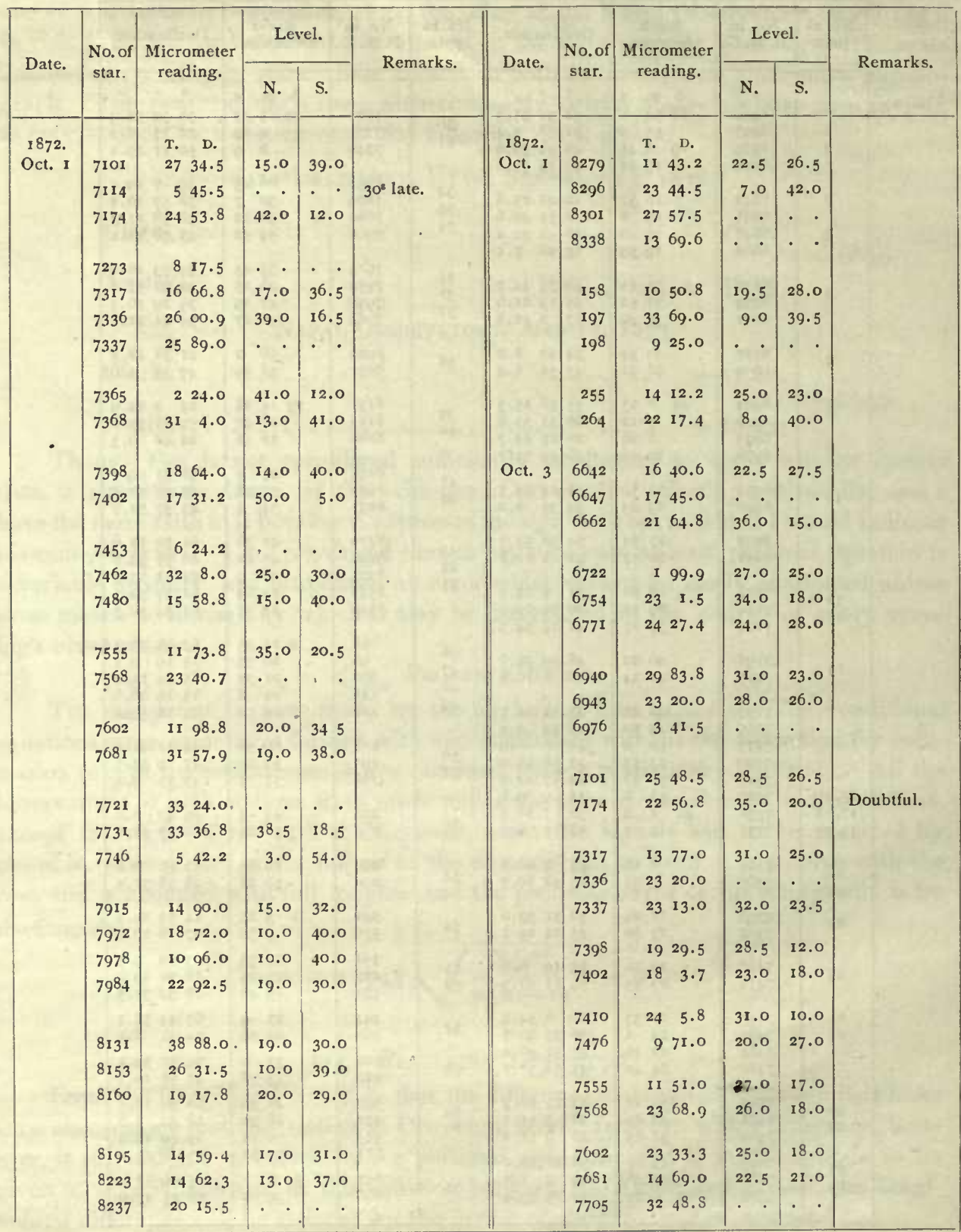


(15.) Reduction of thif Latitude-Observations-Continued.

Observations for Latitude.-Station, Cheyenne, Wyoming Territory.

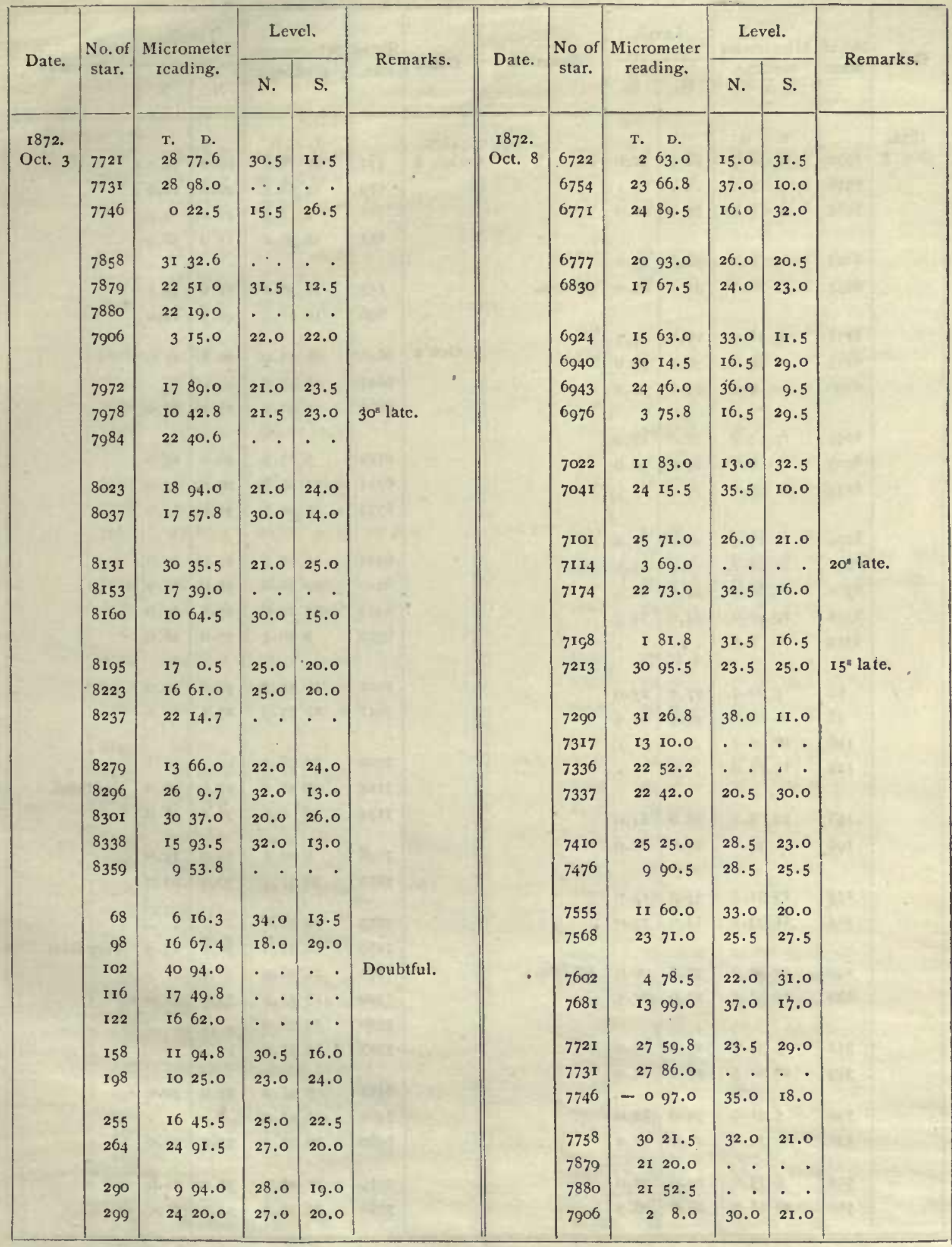


(15.) Reduction of the Latitude-Observations-Continued.

Observations for Latitude.-Station, Cheyenne, Wyoming Tervitory.

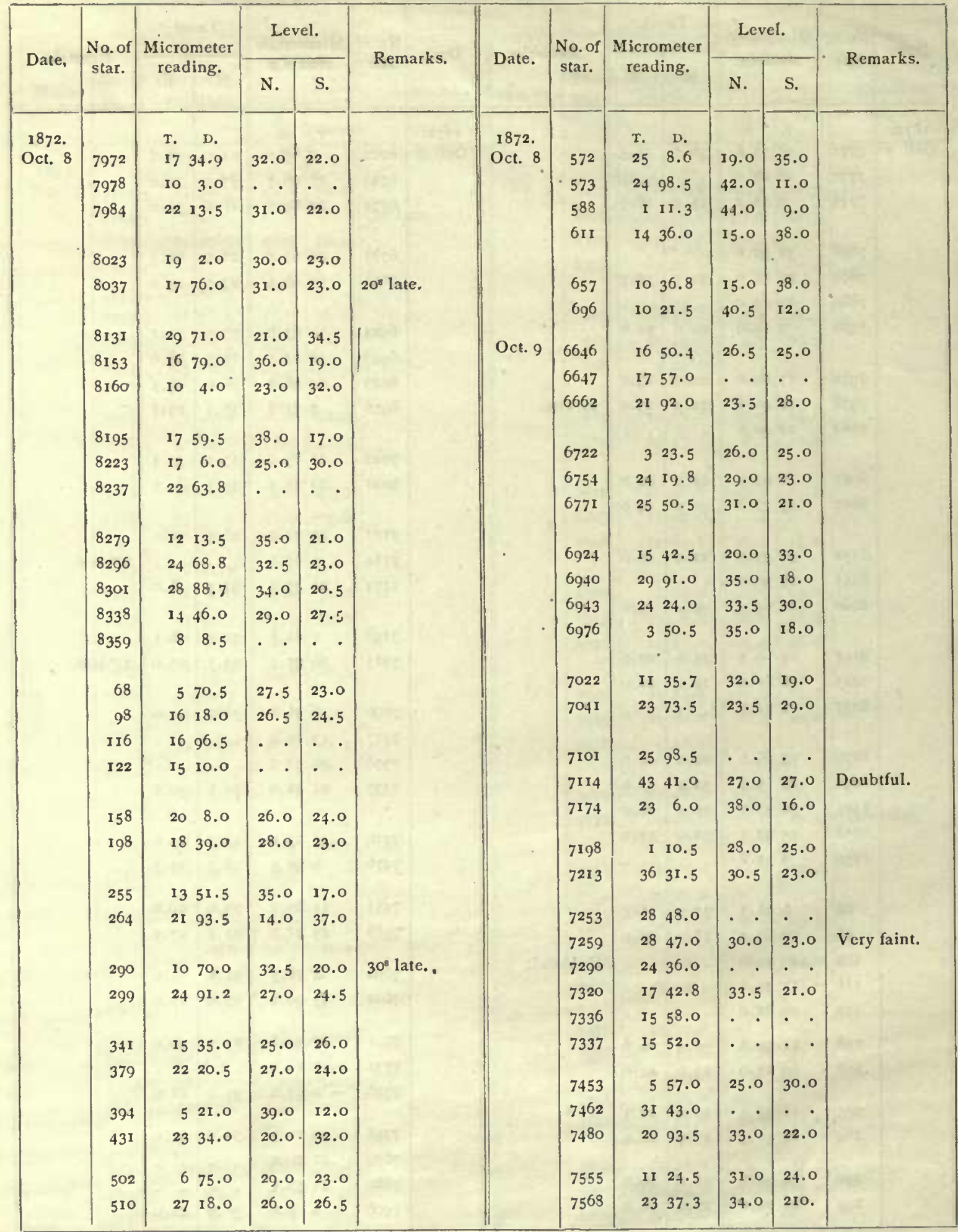


(i 5.) Reduction of tue Latitude-Observations-Continued.

Observations for Latitude.-Station, Cheyenne, Wyoming Tervitory.

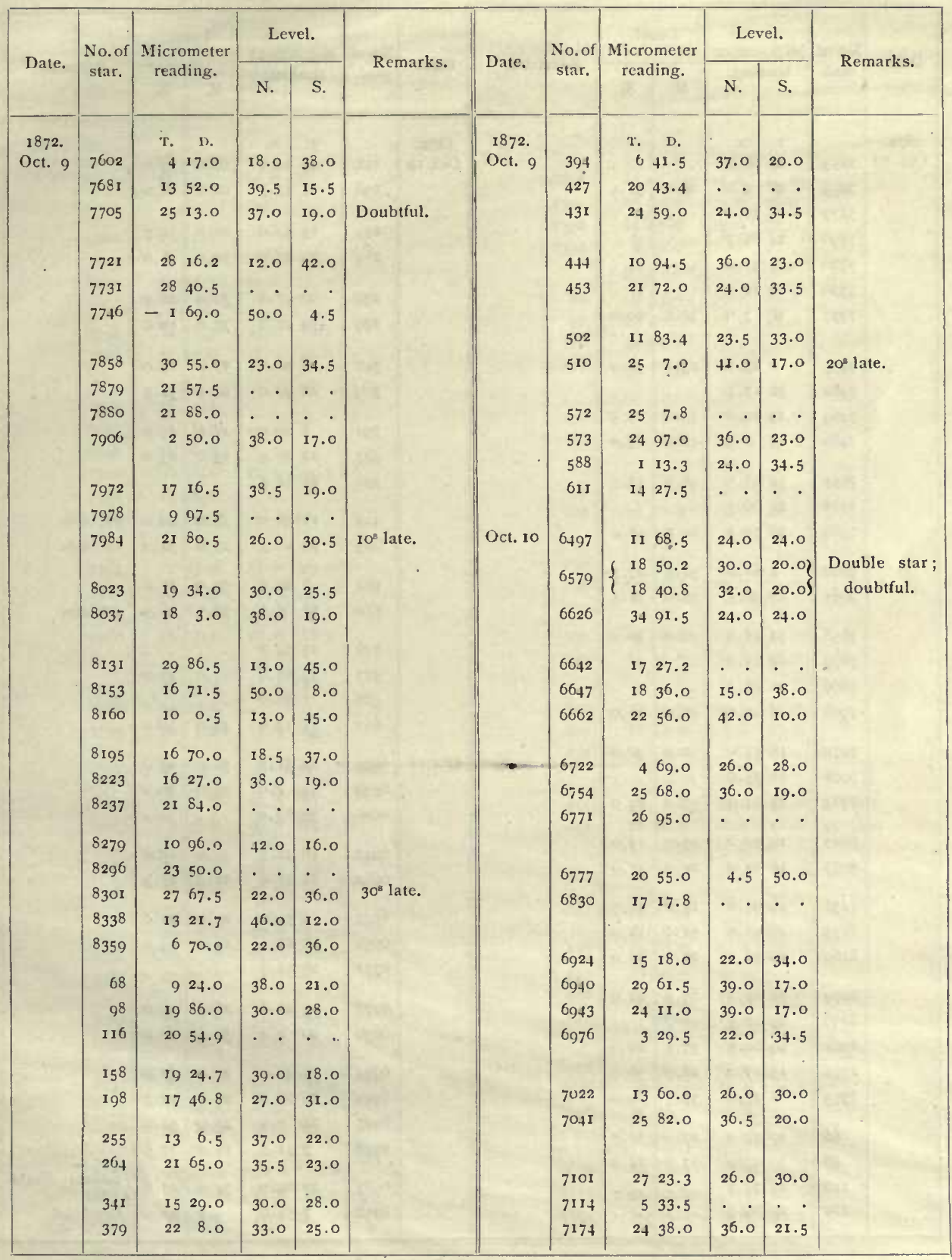


(15.) Reduction of the Latitude-Observations-Continued.

Observations for Latitude._Station, Cheyenne, Wyoming Tervitory.

\begin{tabular}{|c|c|c|c|c|c|c|c|c|c|c|c|}
\hline \multirow{2}{*}{ Date. } & \multirow{2}{*}{$\begin{array}{c}\text { No. of } \\
\text { star. }\end{array}$} & \multirow{2}{*}{$\begin{array}{l}\text { Micrometer } \\
\text { reading. }\end{array}$} & \multicolumn{2}{|c|}{ Level. } & \multirow{2}{*}{ Remarks. } & \multirow{2}{*}{ Date. } & \multirow{2}{*}{$\begin{array}{c}\text { No. of } \\
\text { star. }\end{array}$} & \multirow{2}{*}{$\begin{array}{l}\text { Micrometer } \\
\text { reading. }\end{array}$} & \multicolumn{2}{|c|}{ Level. } & \multirow{2}{*}{ Remarks, } \\
\hline & & & N. & S. & & & & & N. & S. & \\
\hline \multirow[t]{38}{*}{$\begin{array}{l}1872 . \\
\text { Oct. } 10\end{array}$} & 7253 & $\begin{array}{l}\text { T. } \quad \text { D. } \\
2788.8\end{array}$ & . . & . . & & $\begin{array}{l}1872 . \\
\text { Oct. 10 }\end{array}$ & 158 & $\begin{array}{lc}\text { T. } & \text { D. } \\
\text { I9 } & 70.0\end{array}$ & 18.0 & 39.0 & 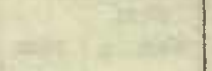 \\
\hline & 7259 & $28 \quad 4.0$ & 26.0 & 31.5 & 1 & & 198 & 1797.5 & 42.0 & 16.0 & \\
\hline & 7273 & -216.0 & .. & . $\cdot$ & & & 255 & 1531.0 & 26.0 & 31.5 & \\
\hline & 7290 & 2485.7 & . . & $\cdot \cdot$ & & & 264 & $\begin{array}{ll}15 & 37.0 \\
23 & 97.0\end{array}$ & 39.0 & $\begin{array}{l}31.5 \\
18.0\end{array}$ & \\
\hline & 7320 & 1791.0 & . $\cdot$ & . $\cdot$ & w & & & & & & \\
\hline & 7336 & $16 \quad 12.5$ & $\cdot \cdot$ & $\cdot \cdot$ & 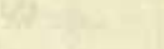 & & 290 & 10 97.8 & 30.0 & 26.5 & \\
\hline & 7337 & $16 \quad 3.0$ & $3^{8} .0$ & 20.0 & & & 299 & $25 \quad 27.7$ & 37.0 & 19.0 & \\
\hline & 7453 & 623.2 & 23.0 & 36.0 & 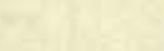 & & $34 I$ & I6 20.0 & 37.0 & 19.0 & \\
\hline & 7462 & $32 \quad 12.3$ & $\cdot \cdot$ & . $\cdot$ & 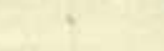 & & 379 & 2292.0 & 24.5 & 32.5 & \\
\hline & 7469 & 1349.0 & 45.0 & 13.5 & & & 394 & 824.0 & 27.5 & 29.0 & \\
\hline & 7480 & 158.3 & 42.0 & 17.0 & & & 427 & $22 \quad 26.9$ & 33.0 & 23.0 & \\
\hline & 7555 & 1248.5 & 46.0 & 12.5 & $10=$ & & $43 \mathrm{I}$ & 2638.5 & $\cdot \cdot$ & . . & \\
\hline & 7568 & 2466.5 & $\cdot \cdot$ & $\cdot$. & & & $4+4$ & II 38.0 & 37.0 & 20.0 & $20^{8}$ late. \\
\hline & 7569 & 2650.2 & 20.5 & 38.0 & & & 453 & 2210.5 & 23.0 & 34.0 & $15^{s}$ late. \\
\hline & 7602 & 481.0 & 31.5 & 27.5 & & & & 621.5 & & 27.0 & \\
\hline & 7681 & 1396.5 & 38.0 & 21.5 & & & $\begin{array}{l}502 \\
510\end{array}$ & 2661.0 & $\begin{array}{l}30.0 \\
32.0\end{array}$ & $\begin{array}{l}27.0 \\
26.0\end{array}$ & I $5^{\mathrm{n}}$ late. \\
\hline & 7858 & 3153.0 & 26.0 & 36.0 & & & & & & & \\
\hline & 7879 & 2285.0 & . . & . . & the & & 572 & 2594.2 & $\cdot \cdot$ & $\cdot \cdot$ & test \\
\hline & 7880 & 2253.5 & . . & . . & th & & 573 & 2581.0 & II.O & 47.0 & \\
\hline & 7906 & 341.0 & 46.0 & 16.0 & 25 & & 588 & I 98.0 & $\cdot$ & $\cdot$ & 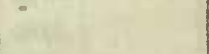 \\
\hline & 7972 & 1857.0 & 32.5 & 30.0 & & & & 1515.0 & 43.0 & 10.0 & \\
\hline & 7978 & II 25.0 & & . . & & Oct. I I & $649 I$ & 1231.5 & 30.0 & 15.0 & 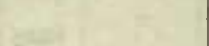 \\
\hline & 7984 & 2324.0 & 33.0 & 29.0 & & & 6579 & I9 4.0 & 25.0 & 20.0 & \\
\hline & 8023 & 1950.0 & 49.0 & 13.0 & & & 6626 & 3563.0 & $\cdot \cdot$ & & \\
\hline & 8037 & 1824.0 & 21.0 & 41.0 & & & $66+2$ & 1651.5 & 35.0 & 13.0 & \\
\hline & & & & & & & 6662 & 2171.8 & 22.0 & $25 \cdot 5$ & $x^{2}=$ \\
\hline & 8131 & 2995.0 & 15.0 & $4^{8.5}$. & & & 6722 & 459.0 & 29.0 & 22.0 & \\
\hline & 8153 & 1691.0 & 51.0 & $12.0^{\circ}$ & & & 6754 & 2587.0 & 17.0 & 34.0 & \\
\hline & 8160 & 10 31.0 & 21.0 & 42.0 & & & 6771 & $269 \mathrm{r} \cdot 7$ & . . & . . & \\
\hline & 8279 & 1287.3 & 29.5 & 28.0 & & & 6777 & 2322.0 & 16.0 & 35.0 & \\
\hline & 8296 & 2542.5 & $\cdot \cdot$ & $\cdot \cdot$ & & & 6830 & $20 \quad 4.4$ & 30.5 & 20.0 & \\
\hline & 8301 & 2966.5 & 37.5 & 20.0 & & & & & & & \\
\hline & 8338 & 1517.0 & 28.0 & 30.0 & & & 6924 & 1540.0 & 21.0 & 32.0 & \\
\hline & 8359 & 877.0 & 37.5 & 20.0 & & & 6940 & 2968.0 & 25.0 & $27 \cdot 5$ & \\
\hline & 68 & I0 47.0 & 27.0 & 31.5 & & & 6943 & $24 \quad 6.0$ & 22.0 & 31.0 & \\
\hline & $9^{8}$ & 2097.0 & $3 \% .0$ & 21.0 & & & 6976 & $35 \mathrm{I} .5$ & 25.0 & $27 \cdot 5$ & \\
\hline & 116 & $21 \quad 74.4$ & . . & . . & & & 6983 & 2761.0 & 34.0 & 17.0 & $\left\{\begin{array}{c}30^{3} \text { late ; doubt- } \\
\text { ful. }\end{array}\right.$ \\
\hline & 122 & 2089.5 & . . & . . & & & 6998 & $75^{8.6}$ & 9.5 & 42.0 & Doubtful. \\
\hline
\end{tabular}


(i 5.) Reduction of the Latitude-Observations-Continued.

Observations for Latitude.-Station, Cheyenne, Wyoming Territory,

\begin{tabular}{|c|c|c|c|c|c|c|c|c|c|c|c|c|c|}
\hline \multirow{2}{*}{ Date. } & \multirow{2}{*}{$\begin{array}{c}\text { No. of } \\
\text { star. }\end{array}$} & \multirow{2}{*}{\multicolumn{2}{|c|}{$\begin{array}{l}\text { Micrometer } \\
\text { reading. }\end{array}$}} & \multicolumn{2}{|c|}{ Level. } & \multirow{2}{*}{ Remarks. } & \multirow{2}{*}{ Date. } & \multirow{2}{*}{$\begin{array}{c}\text { No. of } \\
\text { star. }\end{array}$} & \multirow{2}{*}{\multicolumn{2}{|c|}{$\begin{array}{l}\text { Micrometer } \\
\text { reading. }\end{array}$}} & \multicolumn{2}{|c|}{ Level. } & \multirow{2}{*}{ Remarks. } \\
\hline & & & & N. & S. & & & & & & N. & S. & \\
\hline \multirow[t]{42}{*}{$\begin{array}{l}1872 . \\
\text { Oct. I I }\end{array}$} & 7022 & $\begin{array}{l}\mathrm{T} . \\
\mathrm{I} 2\end{array}$ & $\begin{array}{l}\text { D. } \\
4.0\end{array}$ & 16.0 & $34 \cdot 5$ & & $\begin{array}{l}1872 . \\
\text { Oct. II }\end{array}$ & 8279 & $\begin{array}{l}\text { T. } \\
\text { I2 }\end{array}$ & $\begin{array}{c}\text { D. } \\
62.0\end{array}$ & 37.0 & 23.0 & \\
\hline & 7041 & 24 & 43.0 & 32.0 & 19.5 & & & 8296 & 24 & $95 \cdot 5$ & 15.0 & 45.0 & t \\
\hline & & & & $=$ & $x^{2}$ & & & 8301 & 29 & 10.0 & 35.0 & 25.0 & ty \\
\hline & 7ror & 27 & 51.8 & .. & .. & & & 8338 & $x_{4}$ & 95.0 & 15.0 & 45.0 & \\
\hline & 7114 & 5 & 60.5 & 22.5 & 30.0 & & & 8359 & 8 & 55.0 & $\cdot \cdot$ & $\cdot \cdot$ & \\
\hline & 7174 & 24 & 82.3 & 25.0 & 28.0 & & & 68 & 9 & 93.0 & 39.0 & 21.0 & \\
\hline & $719^{8}$ & o & & & & & & $9^{8}$ & 20 & 20.0 & 13.0 & 48.0 & \\
\hline & 7213 & 35 & $\begin{array}{l}81.5 \\
92.0\end{array}$ & $\begin{array}{l}20.0 \\
31.0\end{array}$ & $\begin{array}{l}33.5 \\
22.0\end{array}$ & & & II 6 & 20 & 96.6 & . $\cdot$ & . . & \\
\hline & & $w^{2}$ & $15 x$ & & & & & 122 & 20 & 12.0 & $\cdot \cdot$ & $\cdot \cdot$ & \\
\hline & 7253 & 28 & 1.5 & $\cdot \cdot$ & $\cdot \cdot$ & & & 158 & 19 & 95.0 & 25.0 & $35 \cdot 5$ & \\
\hline & 7259 & 28 & 6.0 & $\cdot \cdot$ & . $\cdot$ & & 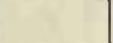 & 198 & 18 & $3 I .0$ & 32.0 & 29.0 & \\
\hline & 7290 & 23 & 86.8 & $\cdot \cdot$ & . $\cdot$ & & & & & & & & 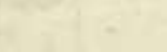 \\
\hline & 7320 & I6 & 73.0 & 20.0 & $35 \cdot 5$ & & & 255 & 14 & 49.5 & 38.0 & 23.5 & 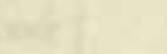 \\
\hline & $\begin{array}{l}733^{6} \\
7337\end{array}$ & I5 & 98.0 &. & $\cdot \dot{ }$ & & & 264 & 22 & 88.2 & 19.0 & 42.0 & \\
\hline & & & 1.0 & & & & & & & & & & \\
\hline & 7453 & 7 & 33.0 & 33.0 & 23.0 & & & 290 & II & 38.8 & 32.0 & 28.5 & \\
\hline & 7462 & 33 & 18.9 & 14.0 & 43.0 & & & 299 & 25 & $45 \cdot 5$ & 23.0 & 35.0 & \\
\hline & 7469 & $r_{4}$ & 84.0 & 14.0 & 43.0 & & & & & & & & \\
\hline & 7480 & 16 & 41.6 & 38.0 & I8.0 & & & $34 \mathrm{I}$ & 15 & 92.0 & 17.0 & 44.0 & \\
\hline & . & 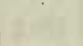 & 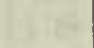 & & wat & w & & 379 & 22 & 95.0 & $34 \cdot 5$ & 26.0 & 17 \\
\hline & 7555 & 12 & 32.0 & 42.0 & 14.0 & & & & & & & & \\
\hline & 7568 & 24 & 20.6 & . . & $\cdot \cdot$ & 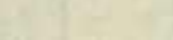 & & 394 & 7 & $2 \pi .0$ & -50.0 & 10.0 & \\
\hline & 7569 & 26 & $5 \cdot 0$ & 7.0 & 49.0 & th & & 427 & 20 & 93.0 & 3.0 & 57.0 & \\
\hline & & 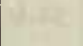 & 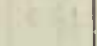 & 10. & & & & 431 & 25 & 5.0 & . : & $\cdot \cdot$ & \\
\hline & 7602 & $\mathrm{I}_{4}$ & 44.0 & 24.0 & 32.0 & & & & & & 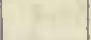 & & \\
\hline & $768 I$ & 23 & 77.8 & 26.0 & $3 \mathrm{r.o}$ & & & 444 & 12 & 6.0 & 39.0 & 22.0 & $30^{\prime}$ late. \\
\hline & 7705 & 2 & 94.0 & 29.0 & 28.0 & & & 453 & 22 & 46.0 & 10.0 & 51.0 & ${ } 5^{\prime \prime}$ late. \\
\hline & 7731 & 33 & 25.2 & 18.5 & 38.0 & & & 502 & 6 & 99.5 & 22.0 & 39.0 & \multirow[b]{2}{*}{$15^{\prime \prime}$ late. } \\
\hline & $774^{6}$ & 4 & 67.9 & $3 \mathrm{r} .0$ & 26.0 & & & 510 & 27 & $45 \cdot 5$ & 34.0 & 27.5 & \\
\hline & 7972 & 18 & $5 \cdot 5$ & 28.5 & 30.0 & & & & & & & & \\
\hline & $797^{8}$ & 10 & 53.5 & 25.0 & 33.0 & & & 572 & 26 & 86.0 & 24.0 & 38.5 & \\
\hline & $79^{8} 4$ & 22 & $6 r \cdot 3$ & $\cdot \cdot$ & .. & & & 573 & 26 & 80.0 & $\cdot \cdot$ & . $\cdot$ & \\
\hline & & & & & & & & $5^{88}$ & 3 & 7.0 & 30.0 & 32.0 & \\
\hline & 8023 & I9 & 99.8 & 31.0 & 27.0 & & & 611 & I 6 & 28.6 & $\cdot \cdot$ & . $\cdot$ & $6=$ \\
\hline & 8037 & r8 & $51 \cdot 5$ & 22.0 & 36.0 & II & & & 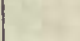 & 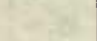 & & - & E \\
\hline & & & & & & to & Oct. ${ }_{2}$ & 6491 & II & $9^{8} .5$ & 38.0 & 15.0 & a \\
\hline & $8 \mathrm{I} 3 \mathrm{I}$ & 23 & 68.0 & 23.0 & 37.0 & & & 6579 & 18 & 84.0 & I2.0 & 42.0 & $13=$ \\
\hline & 8153 & 16 & 89.3 & 28.0 & $3 x \cdot 5$ & & & 6626 & 35 & 39.0 & 15.0 & 41.0 & \\
\hline & 8160 & ro & 2.0 & 25.0 & 35.0 & & & & & & & & \\
\hline & 8195 & 17 & 27.5 & 30.0 & 30.0 & & & 6642 & 15 & 54.0 & 37.0 & I9.0 & \\
\hline & 8223 & 16 & 91.8 & 27.0 & .34 .0 & & & 6647 & 16 & 57.0 & . . & .. & \\
\hline & 8237 & 22 & 48.8 & . $\cdot$ & . $\cdot$ & & & 6662 & 20 & 97.0 & 14.0 & 42.0 & \\
\hline
\end{tabular}


(i 5.) Reduction of the Latitude-Observations-Continued.

Obscrvations for Latitude.-Station, Cheyenne, Wyoming Territory.

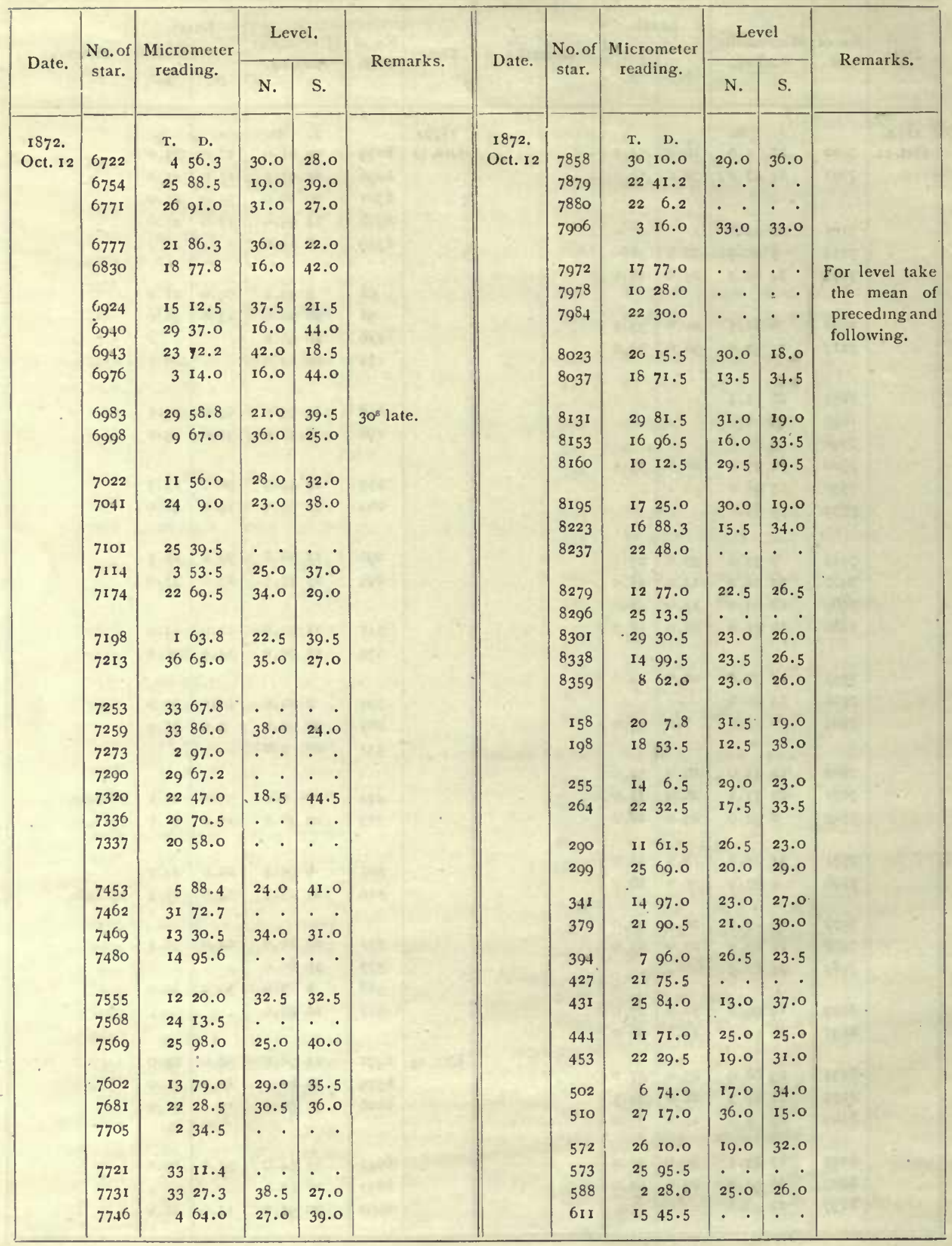


(15.) Reduction of the Latitude-Observations-Continued.

Computations for Latitude of Cheyenne, Wyoming Territory.

\begin{tabular}{|c|c|c|c|c|c|c|c|c|c|c|c|}
\hline \multirow{2}{*}{ Date. } & \multirow{2}{*}{$\begin{array}{l}\text { Number } \\
\text { of pair. }\end{array}$} & \multirow{2}{*}{\multicolumn{3}{|c|}{$\begin{array}{c}\text { Half-sum of dec- } \\
\text { linations. }\end{array}$}} & \multicolumn{4}{|c|}{ Corrections. } & \multirow{2}{*}{\multicolumn{3}{|c|}{ Latitude. }} \\
\hline & & & & & Mic. a & nd ref. & Level. & Meridian. & & & \\
\hline \multirow{27}{*}{$\begin{array}{l}1872 . \\
\text { Oct. }\end{array}$} & & $\circ$ & & " & , & $"$ & $"$ & $" 1$ & 。 & & \\
\hline & 13 & 40 & & 50.5 & +9 & 53.10 & +1.65 & 0.00 & $4 \mathrm{I}$ & 7 & $45 \cdot 3$ \\
\hline & 12 & $4 \mathrm{I}$ & 9 & 12.3 & -1 & 27.24 & +1.65 & 0.00 & & & 46.6 \\
\hline & 17 & & & 57.6 & -9 & 14.28 & $+\circ 8_{3}$ & 0.00 & & & $44 \cdot r$ \\
\hline & 20 & & I 2 & 29.8 & -4 & 46,63 & +0.82 & $0 . \infty 0$ & & & 44.0 \\
\hline & $2 I$ & & 22 & 38.5 & -14 & 55.10 & +0.28 & 0.00 & & & 43.7 \\
\hline & 22 & & 8 & 20.7 & -0 & 41.27 & +5.20 & $0 . \infty 0$ & & & 44.6 \\
\hline & 25 & & 16 & 28.2 & -8 & 32.57 & -8.25 & 0.00 & & & 47.4 \\
\hline & 24 & & 3 & 5.3 & +4 & 50.47 & -8.25 & 0.00 & & & 47.5 \\
\hline & 26 & & 14 & 0.1 & -6 & 2.67 & -8.80 & 0.00 & & & 48.6 \\
\hline & $3 \mathrm{I}$ & & 22 & 35.8 & -14 & 42.54 & -8.50 & $0 . \infty 0$ & & & 44.8 \\
\hline & 30 & & 22 & $3 \mathrm{r} \cdot \mathrm{r}$ & -14 & 35,77 & -8.50 & $0 . \infty 0$ & & & 46.7 \\
\hline & 35 & & & 0.8 & +1 & $5^{8.72}$ & -12.92 & 0.00 & & & 46.6 \\
\hline & 36 & & & $55 \cdot 5$ & +4 & I. 18 & -11.22 & 0.00 & & & $45 \cdot 5$ \\
\hline & 37 & & ro & 7.8 & -2 & 10.69 & -11.20 & 0.00 & & & 45.9 \\
\hline & 39 & & $\mathrm{r}_{4}$ & 27.6 & -6 & 30.52 & -11.00 & $0 . \infty 0$ & & & 46.1 \\
\hline & 40 & & & $\mathrm{r} 5.3$ & +3 & 41.82 & -10.50 & 0.00 & & & 46.6 \\
\hline & $4 I$ & & & 56.2 & +0 & 0.90 & - ro. 45 & 0.00 & & & 46.6 \\
\hline & 42 & & & $3 \cdot 5$ & +2 & 52.84 & -10.40 & 0.00 & & & 45.9 \\
\hline & 43 & & & 10.0 & -6 & 13.36 & -10.70 & 0.00 & & & 45.9 \\
\hline & 45 & & 15 & 10.4 & -7 & $\mathrm{rI}: 36$ & -11.70 & 0.00 & & & $47 \cdot 3$ \\
\hline & 46 & & 13 & 8.4 & -5 & 9.62 & -10.70 & 0.00 & & & 48.1 \\
\hline & 48 & & 13 & 32.6 & -5 & 34.22 & - 10.70 & 0.00 & & & 47.7 \\
\hline & 49 & & & $5 \cdot 3$ & -5 & $5 \cdot \mathrm{II}$ & -10.70 & 0.00 & & & $49 \cdot 5$ \\
\hline & 50 & & 55 & $5^{8} \cdot 3$ & +12 & 0.50 & -10.70 & 0.00 & & & $48 . I$ \\
\hline & $5 \mathrm{I}$ & & 8 & 36.5 & -0 & 39.10 & -10.70 & 0.00 & & & 46.7 \\
\hline & $5^{2}$ & & 12 & $5 \cdot 4$ & -4 & 10.26 & -8.25 & 0.00 & & & 46.8 \\
\hline \multirow[t]{16}{*}{ Oct. 3} & 3 & $4 I$ & 5 & 0.0 & +2 & 42.92 & +4.40 & 0.00 & $4 I$ & 7 & $47 \cdot 3$ \\
\hline & 4 & & 5 & 33.2 & +2 & 10.47 & +4.40 & 0.00 & & & 48.1 \\
\hline & 5 & 40 & 56 & 49.8 & +10 & 53.18 & +4.95 & 0.00 & & & $47 \cdot 9$ \\
\hline & 6 & $4 \mathrm{I}$ & 8 & 22.0 & -0 & 39.13 & +4.00 & 0.00 & & & 46.9 \\
\hline & 9 & & $2 I$ & 22.7 & -13 & 41.23 & +2.80 & 0.00 & & & $44 \cdot 3$ \\
\hline & 9 & & 18 & 28.3 & - ro & 46.02 & +2.30 & 0.00 & & & $45 . \mathrm{I}$ \\
\hline & I2 & & 9 & 12.5 & $-I$ & 30.66 & +2.00 & +0. ro & & & 43.9 \\
\hline & 17 & & 12 & 34.2 & -4 & 53.08 & $+3.9^{8}$ & 0.00 & & & 45.1 \\
\hline & 19 & & 12 & 30.0 & -4 & $50.9 \mathrm{r}$ & +3.98 & 0.00 & & & 43.1 \\
\hline & 22 & & 8 & 21.0 & -0 & 39.10 & +5.91 & 0.00 & & & 47.8 \\
\hline & 23 & & I5 & 40.9 & -7 & 57.04 & +3.85 & 0.00 & & & $47 \cdot 7$ \\
\hline & 26 & & 14 & 0.4 & -6 & 18.52 & +4.95 & 0.00 & & & 46.8 \\
\hline & 28 & & โ2 & 13.3 & -4 & 28.62 & +2.33 & 0.00 & & & 47.0 \\
\hline & $3 \mathrm{I}$ & & 22 & 36.3 & $-r 4$ & $53.7 \mathrm{I}$ & +2.20 & 0.00 & & & 44.8 \\
\hline & $3 r$ & & 22 & 30.8 & -14 & $47 \cdot 36$ & +2.20 & 0.00 & & & 45.6 \\
\hline & 32 & & 22 & 15.0 & -14 & 35.71 & +5.20 & $0 . \infty 0$ & & & $44 \cdot 5$ \\
\hline
\end{tabular}


(15.) Reduction of the Latitude-Observations-Continued.

Computations for Latitude of Cheyenne, Wyoming Territory.

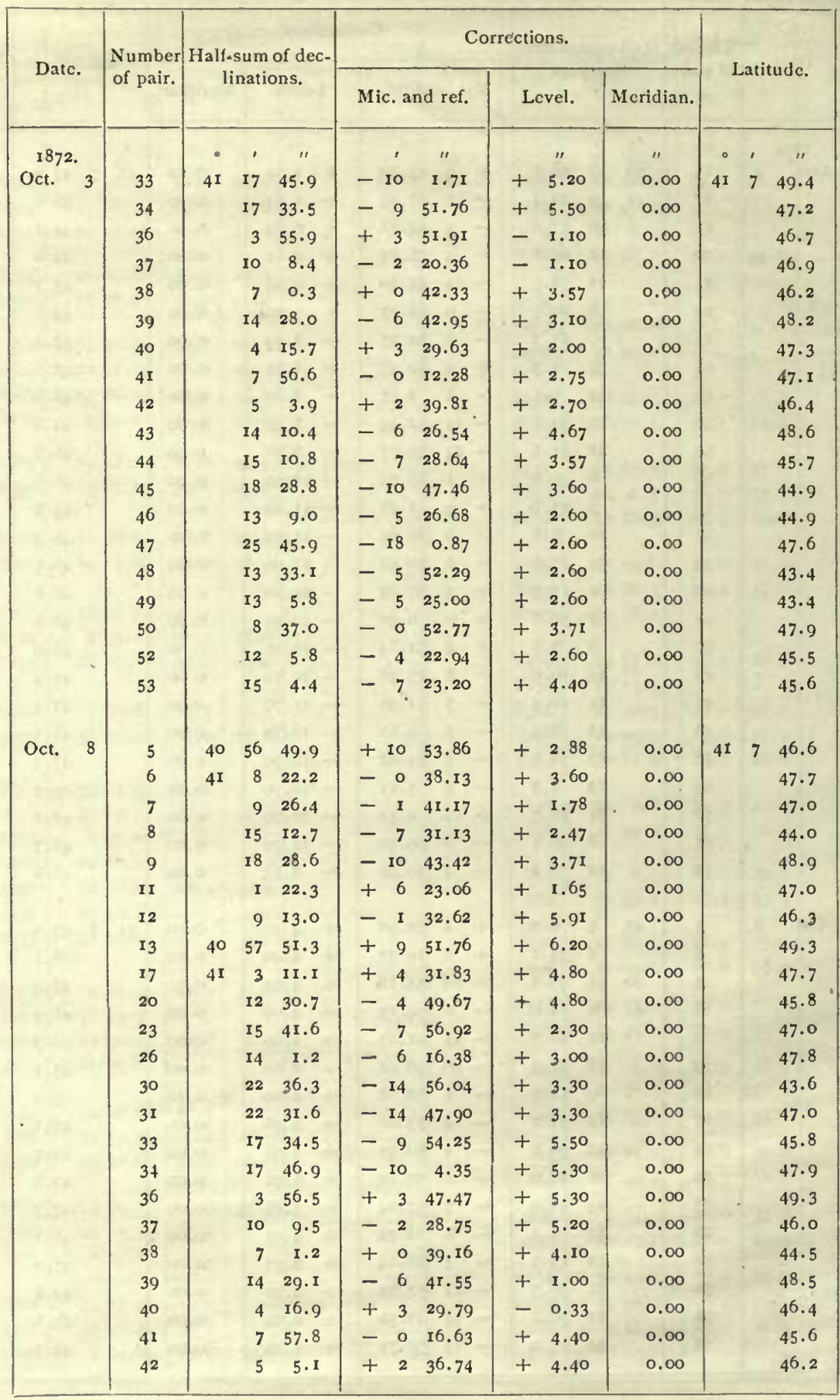


(15.) Rejuction of tue Latitude-Onservations-Continted.

Computations for Latitude of Cheyenne, Wyoming Territory.

\begin{tabular}{|c|c|c|c|c|c|c|c|c|c|c|c|c|c|}
\hline \multirow{2}{*}{ Date. } & \multirow{2}{*}{$\begin{array}{l}\text { Number } \\
\text { of pair. }\end{array}$} & \multirow{2}{*}{\multicolumn{3}{|c|}{$\begin{array}{l}\text { Half-sum of dec- } \\
\text { linations. }\end{array}$}} & \multicolumn{6}{|c|}{ Corrections. } & \multirow{2}{*}{\multicolumn{3}{|c|}{ Latitude. }} \\
\hline & & & & & \multicolumn{2}{|c|}{ Mic, and ref. } & \multicolumn{2}{|c|}{ Level. } & \multicolumn{2}{|c|}{ Meridian. } & & & \\
\hline \multirow{2}{*}{$\begin{array}{l}1872 . \\
\text { Oet. }\end{array}$} & & - & ' & $"$ & , & " & & $"$ & & $"$ & 。 & - & " \\
\hline & 43 & $4^{I}$ & It & II. 5 & -6 & 30.15 & + & 6.50 & & 0.00 & $4 I$ & 7 & 47.8 \\
\hline 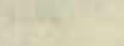 & 44 & & & 42.0 & -7 & 59.49 & + & $3 \cdot 90$ & & 0.00 & & & $4^{6.4}$ \\
\hline in & 45 & & & 30.0 & -10 & 46.53 & & 3. 10 & & 0.00 & & & 46.6 \\
\hline 1 & 46 & & 13 & 10. 2 & -5 & 25.56 & + & 1.80 & & 0.00 & & & 46.4 \\
\hline th & 48 & & & 34.4 & -5 & 49.96 & + & 1.60 & & 0.00 & & & 46.2 \\
\hline 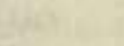 & 49 & & & 7.1 & -5 & 23.10 & + & 1.80 & & 0.00 & & & 45.8 \\
\hline$y^{2}$ & $5 \mathrm{I}$ & & 8 & 38.2 & -0 & 52.52 & + & $2 . \infty 0$ & & 0.00 & & & 47.7 \\
\hline 3 & 52 & & 12 & 7.0 & -4 & 21.69 & - & 1.37 & & 0.00 & & & 43.9 \\
\hline & 53 & & 15 & 5.2 & -7 & 21.71 & + & 4. 10 & + & 0.10 & & & 47.7 \\
\hline & 54 & & 4 & 13.3 & +3 & 33.05 & + & 0.55 & $=$ & 0.00 & & ex & 46.9 \\
\hline & 55 & & I7 & 7.6 & -9 & $23.4^{8}$ & & 4.10 & & 0.00 & & & 48.2 \\
\hline 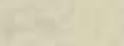 & $5^{8}$ & & 57 & II. 3 & +10 & 34.96 & & I. 50 & 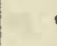 & 0.00 & & & 47.8 \\
\hline F & 59 & & & $5 \cdot 7$ & -12 & 25.08 & & 5.20 & & 0.00 & & & 45.8 \\
\hline & 60 & & 13 & 17.8 & -5 & 30.22 & & 2.20 & & 0.00 & & & 49.8 \\
\hline 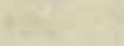 & $6 r$ & & 7 & 50.3 & -0 & 4.75 & + & 1.50 & & 0.00 & & & $47 \cdot 1$ \\
\hline Oct. & 4 & $4 I$ & 5 & 33.3 & +2 & 15.20 & - & 0.90 & & 0.00 & 41 & 7 & 47.6 \\
\hline & 5 & 40 & $5^{6}$ & 49.9 & + in & 51.53 & + & 2.10 & & 0.00 & & & $43 \cdot 5$ \\
\hline 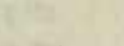 & 6 & 41 & 8 & 22.2 & -0 & 40.62 & + & 5.50 & & 0.00 & & & $47 \cdot \mathrm{T}$ \\
\hline • & 8 & & $x_{5}$ & 12.8 & -7 & 30.19 & + & 1. 10 & & 0.00 & & & 43.7 \\
\hline$x^{2}$ & 9 & & 18 & 28.7 & -10 & 44.44 & + & 2.90 & & 0.00 & & & 47.2 \\
\hline 64 & II & & I & 22.4 & +6 & 24.71 & + & 2. 10 & & 0.00 & & & 49.2 \\
\hline 71 & 14 & & 25 & 58.0 & -18 & $x+.33$ & + & 2.80 & & 0.00 & & & 46.5 \\
\hline at & 15 & & 2 & 1.3 & +5 & 43.50 & + & $5 \cdot 50$ & & 0.00 & & & 50.3 \\
\hline$t^{2}$ & 16 & & $!$ & 55.2 & +5 & 43.18 & + & 5.50 & & 0.00 & & & 43.9 \\
\hline tip & 19 & & 3 & 7.2 & +4 & 34.75 & + & 5.50 & & $0 . \infty$ & & & 47.5 \\
\hline$y^{4}$ & 26 & & 14 & I. 4 & -6 & 16.94 & + & $5 \cdot 50$ & & 0.00 & & & 50.0 \\
\hline$x^{2}$ & 32 & & & 16.1 & $-r_{4}$ & 31.79 & + & 2.60 & & 0.00 & & & 46.9 \\
\hline 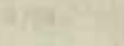 & 33 & & 17 & 34.7 & -9 & 52.85 & + & 2.60 & & $0 . \infty 0$ & & & $44 \cdot 5$ \\
\hline th & 34 & & 17 & 47.1 & -10 & 2.33 & + & 2.60 & & 0.00 & & & 47.4 \\
\hline 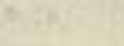 & 36 & & 3 & 56.8 & +3 & $43.4^{6}$ & + & 4.10 & & 0.00 & & & 44.4 \\
\hline in & 37 & & 10 & 9.7 & -2 & 24.21 & + & 4. 10 & & 0.00 & & & 49.6 \\
\hline $5+1$ & 38 & & 7 & 1.4 & +0 & 40.71 & + & 6.30 & & 0.00 & & & 48.4 \\
\hline 15 & 39 & & 14 & 29.3 & -6 & 48.70 & + & 2.80 & & 0.00 & & & 43.4 \\
\hline ras & 41 & & 4 & 17.0 & +3 & 28.55 & + & 2.80 & & 0.00 & & & 48.4 \\
\hline . & 42 & & 7 & 58.1 & -0 & 13.36 & + & 0.10 & & 0.00 & & & $44 \cdot 9$ \\
\hline (1) & 43 & & $5^{\circ}$ & $5 \cdot 3$ & +2 & 39.75 & + & 0.10 & & 0.00 & & & 45.2 \\
\hline 276 & 44 & & 14 & 11.8 & -6 & 29.74 & + & $3 \cdot 30$ & & 0.00 & & & 45.4 \\
\hline 101 & 44 & & 15 & 12.2 & -7 & 29.35 & + & $5 \cdot 50$ & + & 0.10 & & & $4^{8} \cdot 3$ \\
\hline 2,5 & 45 & & 18 & 30.2 & -10 & 51.90 & + & $5 \cdot 50$ & & $0 . \infty 0$ & & & 43.8 \\
\hline 200 & 46 & & 13 & 10.4 & -5 & 30.07 & + & $5 \cdot 30$ & & 0.00 & & & 45.6 \\
\hline Fin & $4^{8}$ & & 13 & 34.7 & - & $51 \cdot 4^{8}$ & + & $5 \cdot 30$ & & 0.00 & & & $4^{8} .5$ \\
\hline 1 & 49 & & 13 & $7 \cdot 4$ & -5 & 25.10 & + & $5 \cdot 30$ & & 0.00 & & & 47.6 \\
\hline
\end{tabular}


(15.) Reduction of the Latipulue-Ohservatrons-Continued.

Computations for Latitude of Cheyene, Wyaming Territory.

\begin{tabular}{|c|c|c|c|c|c|c|c|c|c|c|}
\hline \multirow{2}{*}{ Date. } & \multirow{2}{*}{$\begin{array}{l}\text { Number } \\
\text { of pair. }\end{array}$} & \multirow{2}{*}{\multicolumn{3}{|c|}{$\begin{array}{c}\text { Half-sum of dec- } \\
\text { linations. }\end{array}$}} & \multicolumn{3}{|c|}{ Corrections. } & \multirow{2}{*}{\multicolumn{3}{|c|}{ Latitude. }} \\
\hline & & & & & Mic. and ref. & Level. & Meridian. & & & \\
\hline \multirow{9}{*}{$\begin{array}{l}1872 . \\
\text { Oct. }\end{array}$} & & - & ' & $"$ & or & " & " & 。 & , & " \\
\hline & 51 & $4 I$ & & 38.5 & $-\quad 055.29$ & +4.67 & $0 . \infty 0$ & 41 & 7 & 47.9 \\
\hline & $5^{2}$ & & 12 & $7 \cdot 3$ & -426.82 & +7.60 & 0.00 & & & 45.1 \\
\hline & 54 & & 4 & 13.4 & +331.03 & +2.75 & 0.00 & & & 47.2 \\
\hline & 55 & & 14 & 59.5 & -715.71 & +1.80 & 0.00 & & & 45.6 \\
\hline & 56 & & 17 & 7.8 & -924.88 & +1.79 & 0.00 & & & 44.7 \\
\hline & 57 & & & 17.4 & -534.89 & +1.00 & 0.00 & & & 43.5 \\
\hline & 60 & & 20 & 10.1 & $-\mathrm{I} 2 \quad 24.2 \mathrm{I}$ & +0.70 & 0.00 & & & 46.6 \\
\hline & 59 & & I 3 & IS.O & -532.40 & +0.70 & $0 . \infty 0$ & & & $4^{6.3}$ \\
\hline \multirow[t]{34}{*}{ Oct. 10} & 1 & 40 & 55 & 42.9 & +121.99 & +3.30 & 0.00 & $4 I$ & 7 & $\$ 8.2$ \\
\hline & 4 & 41 & 5 & 33.2 & +210.54 & +2.47 & 0.00 & & & $4^{6.2}$ \\
\hline & 5 & 40 & 56 & $49 \cdot 9$ & +1052.37 & +4.10 & 0.00 & & & $4^{6.4}$ \\
\hline & 6 & 41 & 8 & 22.2 & - o 39.47 & +4.10 & 0.00 & & & 46.8 \\
\hline & 7 & & 9 & 26.4 & -144.80 & +4.10 & 0.00 & & & $45 \cdot 7$ \\
\hline & 8 & & 15 & 12.8 & -728.65 & +2.60 & 0.00 & & & 46.8 \\
\hline & 9 & & 18 & 28.8 & - 10 46.93 & +2.60 & 0.00 & & & $44 \cdot 5$ \\
\hline & II & & I & 22.5 & +619.80 & +3.40 & 0.00 & & 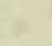 & $45 \cdot 7$ \\
\hline & 12 & & 9 & 13.2 & $-\quad 128.67$ & +2.86 & 0.00 & & & $47 \cdot 4$ \\
\hline & I3 & 40 & 57 & $51 \cdot 5$ & +951.92 & +2.90 & $0 . \infty 0$ & & & 46.3 \\
\hline & 15 & 41 & 2 & I. 4 & +541.22 & +3.40 & $0 . \infty 0$ & & & 46.0 \\
\hline & 16 & & I & $57 \cdot 3$ & $+\quad 545.94$ & +3.40 & 0.00 & & & 46.6 \\
\hline & I7 & & 3 & 11.3 & +431.39 & +3.40 & 0.00 & & & 46.1 \\
\hline & 25 & & 16 & 29.6 & -849.60 & +5.10 & 0.00 & & & 45.1 \\
\hline & 26 & & 14 & 1.5 & -618.55 & +4.40 & 0.00 & & & $47 \cdot 3$ \\
\hline & 33 & & I 7 & 47.2 & -.10 4.19 & +5.50 & $0 . \infty 0$ & & & 48.5 \\
\hline & 34 & & 17 & 34.8 & -954.40 & +5.50 & 0.00 & & & $45 \cdot 9$ \\
\hline & 36 & & 3 & $57 \cdot 5$ & +347.51 & +1.80 & 0.00 & & & 46.8 \\
\hline & 37 & & 10 & 9.8 & -225.14 & +1.78 & 0.00 & & & 46.4 \\
\hline & 38 & & 7 & 1.6 & +039.16 & +4.40 & 0.00 & & & 45.2 \\
\hline & 39 & & 14 & 29.5 & -645.53 & +1.50 & 0.00 & & & $45 \cdot 5$ \\
\hline & 40 & & 4 & 17.2 & $+\quad 325.13$ & +4.90 & 0.00 & & & 47.2 \\
\hline & 43 & & 14 & 12.0 & -630.12 & +5.30 & 0.00 & & & 47.2 \\
\hline & 44 & & 15 & 12.4 & -730.50 & +4.30 & 0.00 & & & 46.2 \\
\hline & 45 & & 18 & 30.4 & - 1049.42 & +4.30 & 0.00 & & & $45 \cdot 3$ \\
\hline & 46 & & 13 & 10.7 & $-\quad 526.34$ & +3.20 & 0.00 & & & 47.6 \\
\hline & 48 & & 13 & $34 \cdot 9$ & -550.40 & +3.20 & 0.00 & & & 47.7 \\
\hline & 49 & & I3 & 7.6 & -524.01 & +3.20 & 0.00 & & & 46.8 \\
\hline & 50 & & 8 & 38.7 & $-053.6 \mathrm{I}$ & +1.38 & 0.00 & & & 46.5 \\
\hline & 52 & & 12 & $7 \cdot 5$ & $-\quad 428.22$ & +4.20 & 0.00 & & & 43.5 \\
\hline & 53 & & 15 & $5 \cdot 5$ & -724.41 & +5.94 & 0.00 & & & 47.0 \\
\hline & 54 & & 4 & 13.4 & +328.86 & +2.75 & $0 . \infty 0$ & & & 45.0 \\
\hline & 55 & & I4 & 59.7 & -716.02 & +2.33 & 0.00 & & & 46.0 \\
\hline & 56 & & I7 & 8.0 & -923.95 & +2.30 & 0.00 & & & $4^{6.4}$ \\
\hline
\end{tabular}


(15.) Reduction of the Latitude-Observations-Continued.

Compututions for Latitude of Cheyenne, Wyoming Territory.

\begin{tabular}{|c|c|c|c|c|c|c|c|c|c|c|c|}
\hline \multirow{2}{*}{ Date. } & \multirow{2}{*}{$\begin{array}{l}\text { Number } \\
\text { of pair. }\end{array}$} & \multirow{2}{*}{\multicolumn{3}{|c|}{$\begin{array}{l}\text { Half-sum of dec- } \\
\text { linations. }\end{array}$}} & \multicolumn{5}{|c|}{ Corrections. } & \multirow{2}{*}{\multicolumn{2}{|c|}{ Latitude. }} \\
\hline & & & & & \multicolumn{2}{|c|}{ Mic. and ref. } & \multicolumn{2}{|c|}{ Level. } & Meridian. & & \\
\hline I 872 & & - & 1 & $"$ & ' & " & & " & $"$ & - & * \\
\hline Oct. 10 & 57 & $4 \mathrm{I}$ & 13 & 17.6 & -5 & 33.33 & + & 1.65 & 0.00 & 417 & $45 \cdot 9$ \\
\hline & 58 & 40 & 57 & II. 5 & +10 & 33.88 & + & $1 \cdot 30$ & 0.00 & & 46.7 \\
\hline & 60 & $4 I$ & 20 & 10.5 & -12 & 24.74 & + & 0.60 & 0.00 & & 46.4 \\
\hline & 59 & & 13 & IS. 2 & -5 & $31 \cdot 31$ & + & 0.55 & 0.00 & & 47.4 \\
\hline Oct. I I & $x$ & 41 & 4 & 12.1 & +3 & 29.01 & $\dot{T}$ & 5.50 & 0.00 & 417 & 46.6 \\
\hline & 2 & 40 & 55 & 42.9 & $+I I$ & 58.41 & + & 5.50 & 0.00 & & 46.8 \\
\hline & 3 & $4 \mathrm{I}$ & 5 & 0.0 & +2 & $4 \mathrm{I} \cdot 7 \mathrm{I}$ & 4 & 5.08 & 0.00 & & 46.8 \\
\hline & 5 & 40 & 56 & 50.0 & +11 & $1 \cdot 38$ & - & 2.75 & 0.00 & & 48.6 \\
\hline & 6 & 41 & $s$ & $22 \cdot 3$ & -0 & 32.54 & - & 2.70 & 0.00 & & 47.1 \\
\hline & 7 & & 9 & 26.4 & -1 & $38.7 \mathrm{I}$ & - & 2.33 & 0.00 & & $45 \cdot 4$ \\
\hline & 8 & & 15 & 12.9 & -7 & 23.8 .2 & - & 3.74 & 0.00 & & $45 \cdot 3$ \\
\hline & 9 & & IS & 28.9 & -10 & 38.54 & - & 3.20 & 0.00 & & 47.2 \\
\hline 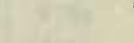 & 10 & 40 & 57 & 30.0 & +10 & 22.34 & - & 4.40 & 0.00 & & 48.0 \\
\hline & II & 41 & I & 22.5 & +6 & 25.08 & - & I. 65 & 0.00 & & 45.9 \\
\hline & I 2 & & 9 & 13.3 & $-I$ & 23.76 & - & 2.90 & 0.00 & & 46.6 \\
\hline & 13 & 40 & 57 & 51.6 & +9 & $57 \cdot 30$ & - & 2.88 & 0.00 & & 46.0 \\
\hline & 14 & $4 I$ & 25 & 58.2 & -18 & 11.06 & - & 1.23 & 0.00 & & 45.9 \\
\hline & 15 & & 2 & 1.5 & +5 & 50.74 & $\rightarrow$ & 3.40 & 0.00 & & 48.8 \\
\hline & 16 & & $\mathbf{I}$ & 57.4 & +5 & 52.14 & - & 3.40 & 0.00 & & 46.1 \\
\hline & I8 & & 3 & I I.4 & +4 & 36.26 & - & 3.40 & 0.00 & & $44 \cdot 3$ \\
\hline 21 & 24 & & 3 & 6.8 & +4 & 42.39 & - & 2.40 & 0.00 & & $4^{6} .8$ \\
\hline & 26 & & 14 & I. 7 & -6 & 9.42 & - & 3.80 & 0.00 & & 48.5 \\
\hline & $3 I$ & & 22 & 37.7 & -14 & 48.05 & - & $3.9^{8}$ & $0 . \infty 0$ & & $45 \cdot 7$ \\
\hline & 36 & & 10 & I0.0 & -2 & 21.66 & - & 2.60 & 0.00 & & $45 \cdot 7$ \\
\hline & $3^{8}$ & & 7 & 1.7 & +0 & 46.09 & - & 2.75 & 0.00 & & 45.0 \\
\hline & 39 & & I4 & 29.8 & -6 & 37.42 & - & 4.80 & 0.00 & & 47.6 \\
\hline & 40 & & 4 & I 7.4 & +3 & $33.6 \mathrm{r}$ & - & 3.70 & 0.00 & & $47 \cdot 5$ \\
\hline & $4 I$ & & 7 & $5^{8.6}$ & +0 & II.IO & - & 2.00 & 0.00 & & $45 \cdot 5$ \\
\hline te & $4^{2}$ & & 5 & 5.8 & +2 & 42.02 & - & 2.00 & 0.00 & & 45.8 \\
\hline & 43 & & 14 & 12.3 & -6 & 23.37 & - & $4 \cdot 40$ & 0.00 & & 44.5 \\
\hline & $4 t$ & & 15 & 12.7 & -7 & 19.78 & - & $5 \cdot 50$ & 0.00 & & 47.4 \\
\hline & 45 & & 18 & 30.7 & -10 & 38.69 & - & $5 \cdot 50$ & 0.00 & & 46.5 \\
\hline & $4^{6}$ & & 13 & 10.9 & -5 & 19.19 & - & 4.67 & 0.00 & & 47.0 \\
\hline & $4^{3}$ & & 13 & 35.2 & -5 & 43.00 & - & 4.60 & 0.00 & & 47.6 \\
\hline & 49 & & 13 & 7.9 & -5 & 16.70 & - & 4.60 & 0.00 & & 46.6 \\
\hline & 51 & & 9 & 39.0 & -0 & 50.97 & - & 2.00 & 0.00 & & 46.0 \\
\hline & $5^{2}$ & & 12 & $7 \cdot 7$ & -4 & 20.67 & - & 2.33 & 0.00 & & 44.7 \\
\hline & 53 & & 15 & 5.8 & - & 17.20 & - & 2.33 & 0.00 & & 46.3 \\
\hline & 54 & & 4 & 13.4 & +3 & 38.49 & $\rightarrow$ & 5.08 & 0.00 & & 46.8 \\
\hline & 55 & & I5 & 0.0 & -7 & 6.42 & - & 3.85 & $0 . \infty 0$ & & 49.7 \\
\hline & 56 & & 17 & 8.2 & -9 & 14.47 & & 3.80 & 0.00 & & $49 \cdot 9$ \\
\hline & 57 & & 13 & I 7.8 & -5 & 23.24 & - & 6.60 & 0.00 & & 48.0 \\
\hline
\end{tabular}


(15.) Reduction of mif La'titudi-Olisharations-Continued.

Computations for Latitude of Cheycnne, Wyoming Territory.

\begin{tabular}{|c|c|c|c|c|c|c|c|c|c|c|c|c|c|}
\hline \multirow{2}{*}{ Date. } & \multirow{2}{*}{$\begin{array}{l}\text { Number } \\
\text { of pair. }\end{array}$} & \multirow{2}{*}{\multicolumn{3}{|c|}{$\begin{array}{l}\text { Half-sum of dec- } \\
\text { linations. }\end{array}$}} & \multicolumn{6}{|c|}{ Corrections. } & \multirow{2}{*}{\multicolumn{3}{|c|}{ Latitude. }} \\
\hline & & & & & \multicolumn{3}{|c|}{ Mic. and ref. } & \multicolumn{2}{|c|}{ Level. } & Meridian. & & & \\
\hline 1872. & & - & ' & ", & & . & $"$ & & $"$ & $"$ & 。 & . & " \\
\hline Oct. II & 58 & 40 & 57 & 11.7 & + & 10 & 35.90 & - & 2.90 & $0 . \infty$ & $4 I$ & 7 & 44.7 \\
\hline 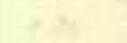 & 60 & 41 & 20 & 10.5 & - & 12 & 19.39 & - & 4.53 & 0.00 & & & 46.6 \\
\hline$\cdot$ & 59 & & 13 & 18.4 & - & 5 & 26.77 & - & 4.50 & $0 . \infty 0$ & & & 47.1 \\
\hline Oct. 12 & 2 & 40 & 55 & 42.9 & + & -12 & $7 \cdot 43$ & - & 0.90 & $0 . \infty 0$ & 41 & 7 & 49.4 \\
\hline & 3 & $4 I$ & 5 & O.I & & - 2 & 48.76 & - & 2.75 & 0.00 & & & 46.1 \\
\hline$\sqrt{2}$ & 4 & 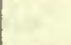 & 5 & $33 \cdot 3$ & & 2 & 16.75 & - & 2.70 & 0.00 & & & $47 \cdot 3$ \\
\hline 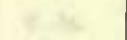 & 5 & 40 & 56 & 50.0 & & II & 2.69 & - & . $5.00^{\circ}$ & 0.00 & & & 47.7 \\
\hline 3. & 6 & 41 & 8 & 22.3 & & 0 & 31.86 & - & 4.40 & 0.00 & & & 46.0 \\
\hline$x_{x}=$ & 7 & $b^{2}$ & 9 & 26.4 & & 1 & 35.88 & - & $3 \cdot 30$ & 0.00 & & & 47.2 \\
\hline & 8 & & 15 & 13.0 & - & 7 & 22.73 & - & $3 \cdot 30$ & 0.00 & & & 47.0 \\
\hline 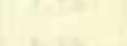 & 9 & & 18 & 28.9 & & 10 & 39.69 & - & 1.23 & 0.00 & & & 48.0 \\
\hline h the & 10 & 40 & 57 & 30.0 & & 10 & 19.05 & - & 2.20 & +0.10 & & & 47.0 \\
\hline 27 & 11 & $4 I$ & I & 22.6 & & 6 & 29.43 & - & $5 \cdot 40$ & 0.00 & & & 46.6 \\
\hline & 12 & 2 & 9 & 13.4 & & I & 23.92 & - & 2.20 & 0.00 & & & 47.3 \\
\hline & 13 & 40 & 57 & 51.7 & & g & 55.49 & - & 2.00 & 0.00 & & & 45.2 \\
\hline$x^{2}$ & 14 & 41 & 25 & $5^{8} .3$ & - & 18 & 8.17 & - & - 2.47 & 0.00 & & & 47.6 \\
\hline$x^{2}$ & 15 & - & 2 & 1.6 & + & 5 & 48.34 & - & 3.30 & 0.00 & & & 46.6 \\
\hline 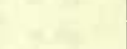 & 16 & & 1 & 57.5 & + & 5 & 54.00 & - & 3.30 & 0.00 & & & 48.2 \\
\hline & 17 & & 16 & 58.9 & - & -9 & 11.20 & - & 3.30 & 0.00 & & & 44.4 \\
\hline & 19 & & 3 & 7.4 & + & -4 & 42.58 & - & 3.30 & 0.00 & & & 46.7 \\
\hline & 24 & & 3 & 6.9 & + & -4 & 41.96 & - & - 3.80 & 0.00 & & & 45.1 \\
\hline & 20 & & 14 & 1.8 & - & -6 & 10.94 & - & 4.40 & 0.00 & & & $4^{6.5}$ \\
\hline & 28 & t & 12 & 14.9 & - & -4 & 24.02 & - & . 3.30 & 0.00 & & & 47.6 \\
\hline & 30 & 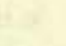 & 22 & 33.2 & - & -14 & 44.97 & - & 0.10 & 0.00 & & & 48.1 \\
\hline & 31 & & 22 & 37.9 & - & -14 & 49.91 & - & 0.10 & 0.00 & & & 47.9 \\
\hline & 32 & & 22 & 16.6 & - & -14 & 28.39 & - & 1.90 & 0.00 & & & 46.3 \\
\hline & 33 & 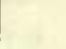 & 17 & 47.6 & - & -9 & $5^{8} .35$ & - & 2.00 & 0.00 & & & 47.2 \\
\hline & 34 & & 17 & 35.2 & - & -9 & 47.47 & - & 2.00 & $0 . \infty 0$ & & & 45.7 \\
\hline & 36 & - & 3 & 57.8 & + & -3 & 52.79 & - & 2.20 & $0 . \infty 0$ & & & $4^{8} .4$ \\
\hline & 37 & & 10 & 10.2 & - & -2 & 20.79 & - & 2.20 & $0 . \infty$ & & & 47.2 \\
\hline || & 38 & & 7 & 2.0 & + & 0 & 44.75 & - & $=2 .+7$ & 0.00 & & & $44 \cdot 3$ \\
\hline (x) & 39 & 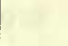 & 14 & 29.9 & - & -6 & $39 \cdot 3^{8}$ & - & 1.50 & 0.00 & & & 49.0 \\
\hline 46 & 40 & tat & 4 & 17.6 & + & - 3 & 32.59 & - & . 2.20 & $0 . \infty 0$ & & & $4^{8} .0$ \\
\hline $1+5$ & 41 & & 7 & 58.8 & - & 0 & 11.41 & - & 2.10 & 0.00 & & & $45 \cdot 3$ \\
\hline - & 42 & & 5 & 6.0 & + & 2 & 42.55 & - & 2. 10 & 0.00 & & & 46.5 \\
\hline & 43 & & 14 & 12.5 & - & 6 & 24.30 & - & - 1.90 & 0.00 & & & $4^{6.3}$ \\
\hline & 44 & & 15 & 12.9 & - & 7 & 24.75 & - & $=1.70$ & $0 . \infty$ & & & 40.4 \\
\hline & 45 & & 18 & 30.9 & - & ro & 42.89 & - & $=1.70$ & 0.00 & & & 46.3 \\
\hline & 50 & & 9 & 39.2 & - & 0 & $47.9^{6}$ & - & . 3.57 & 0.00 & & & 47.7 \\
\hline & 52 & & 12 & 7.9 & - & - 4 & 16.72 & - & 2.75 & 0.00 & & & 48.4 \\
\hline 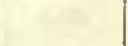 & 53 & & 15 & 6.0 & - & 7 & 17.45 & - & . 1.54 & $0 . \infty 0$ & & & 47.0 \\
\hline - & 54 & I & 4 & 13.4 & + & 3 & 35.54 & - & 3.57 & 0.00 & & & 45.4 \\
\hline & 55 & 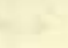 & 15 & 0.2 & - & -7 & $7 \cdot 77$ & - & 5.80 & 0.00 & & & 45.6 \\
\hline & 56 & & 17 & 8.4 & - & -9 & 15.73 & - & 5.77 & 0.00 & & & 46.9 \\
\hline & 57 & & 13 & 18.0 & & -5 & 28.98 & - & - 3.30 & 0.00 & & & $45 \cdot 7$ \\
\hline & 58 & 40 & 57 & 11.8 & & -10 & 34.96 & + & . 1.10 & 0.00 & & & 47.9 \\
\hline & 60 & 41 & 20 & 10.8 & & -12 & 20.33 & - & 3.85 & 0.00 & & & $4^{6.6}$ \\
\hline
\end{tabular}


(15.) Oisservations for Latitude, Cheyenne, Wyoming Territory.

\section{Recapitulation.}

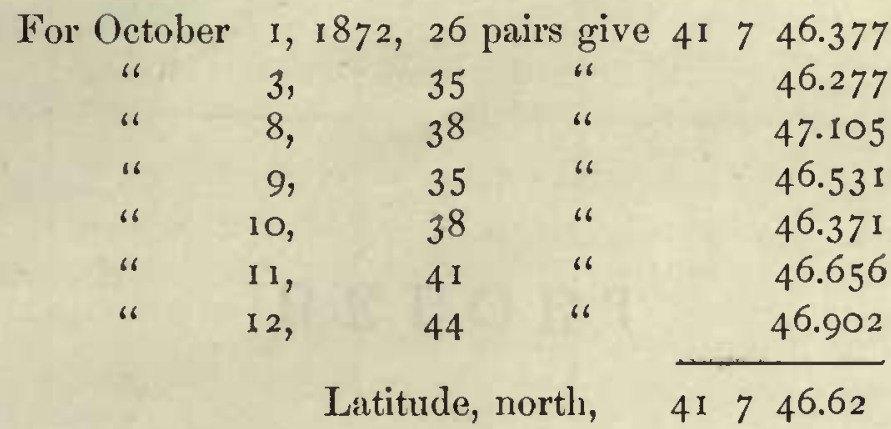

Giving the first series half-weight on account of the smaller number of the observations and the less favorable condition of that night's work, the resulting latitude, and the one adopted for this station, is, $41^{\circ} 7^{\prime} 46^{\prime \prime} .62$, with a probable error of $\pm 0^{\prime \prime} .08$. The latitudes were originally computed by Professor Willian A. Rogers, of Cambridge, Mass., and revised by Dr. F. Kampf.

\section{(16.) Resulting Astronomical Co-ordinates.}

Taking the longitnde of the Salt Lake observatory to be $2^{\mathrm{h}} 19^{\mathrm{m}} 22^{\mathrm{s}} .74$ west of Washington by deternination of the United States Coast Survey, and Washington to be $5^{\mathrm{h}} 8^{\mathrm{m}} 12^{8}$.I 2 west of Greenwich according to the report of Rear-Admiral B. F. Sands, Superintendent of the United States Naval Observatory, October 6, I87r, Cheyenne is in longitude west from Washington, in time, $\mathrm{I}^{\mathrm{h}} 5 \mathrm{I}^{\mathrm{m}} 3^{8} \cdot 3 \mathrm{O}$; in arc, $27^{\circ} 45^{\prime} 49^{\prime \prime} .50$; in longitude west from Greenwich, in time, $6^{\mathrm{b}} 59^{\mathrm{m}} 15^{\mathrm{s}} \cdot 4^{2}$; in are, $104^{\circ} 48^{\prime} 5 \mathrm{I}^{\prime \prime} .30$; in latitude, north, $4 \mathrm{I}^{\circ} 7^{\prime} 46^{\prime \prime} .62 \pm \mathrm{O}^{\prime \prime} .08$.

This final result for longitude is subject, as already stated, to a correction for the personal equation of the observers. It is possible, also, that the longitude of Salt Lake may be changed when the observations made last October at Detroit and Ogden by the United States Lake Survey and your expedition respectively are computed. In such an event, of course the longitude of Cheyenne will be correspondingly affected.

Respectfully, yours,

Lieut. Geo. M. Whesler,

JOHN H. CLARK.

Corps of Engineers, in charge. 



\section{R E P O R'T}

os

\section{ASTRONOMICAL OPERATIONS,}

CONDUC'TED DUIRING

\section{THE FIELD-SEASON OF 1873 ,}

$\mathbf{A T}$

TIIE MAIN OR PRINARY FIELD-STATION, COLORADO SPRINGS, COLORADO TERRITORY,

AND

DEIUCTION OF RESUL'PS.

BY

Dr. F. KAMPF,

CIVILIAN ABTRONOMICAL ABSIBTANT. 


\subsection{9

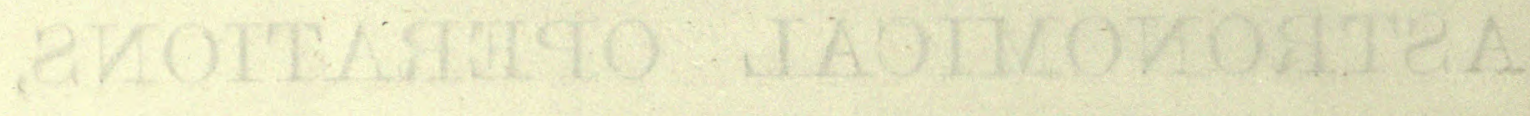

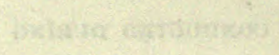

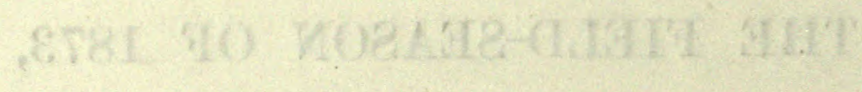

74

सं.

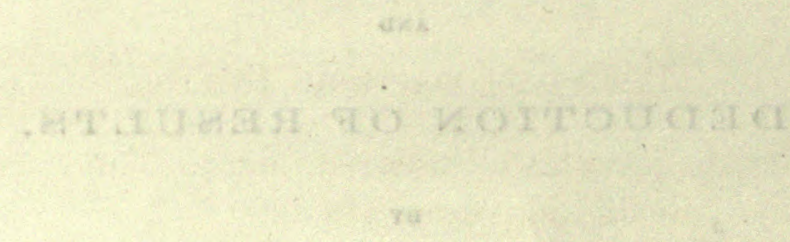

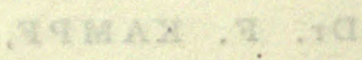

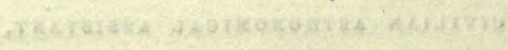




\section{U. S. Engineer Office, Geographical and Geolongical Explorations and Surveys West of iooth Meridian, Washington, D. C., Jamuary I, I 874 .}

SIR: There is presented herewith a report upon the astronomieal observations taken by myself, and the party under my charge, at Colorado Springs, Colorado Territory, during the field-season of 1873 .

\section{Geograpitical Position of Station. \\ Longitude $=104^{\circ} 49^{\prime}$ i $5^{\prime \prime}$. 10 .$$
\text { Latitude }=38^{\circ} 49^{\prime} 4 \mathrm{I}^{\prime \prime} .67 \text {. }
$$

Colorado Springs is a town in El Paso County, Colorado Territory. It has been built up within five years, and has nearly fifteen hundred inhabitants, and the place promises to become one of considerable importance. During the summer-months the hotels (of which there are quite a large number) are filled with invalids, who flock here on aceount of the beautiful scenery and the salubrity of the climate. The track of the Denver and Rio Grande Railway passes around the town at a distance from the town-limits of about four thousand feet.

The astronomical point is situated between the town and the railroad, about six liundred and fifty feet distant from the latter, on a slight eminenee near the freightdepot of the Denver and Rio Grande Railroad. The observations were conducted on a pier built of bricks, which was replaced two months later by a solid sandstone monument, furnished by Mr. S. G. Ward, of Pueblo.

\section{Physical-Geography Details.}

This part of Colorado is not well watered, but the land where irrigated yields almost in every instance splendid larvests.

From the astronomical point there is a clear outlook to the uortl, south, and east. Looking west, prominent peaks and foot-hills of the Rocky Mountain range are seen running nortl and south; Pike's Peak, immediately west, being the highest, and Cheyenne Mountain the highest in the south-southwest. At the foot of Cheyenne Mountain there is a creek, the waters of which are brought, by means of ditches, to Colorado Springs. From the station the plains rise a little to the east, at the horizon say one hundred and fifty feet. In the southeast there is a hill about four hundred feet high, called Washington Mountain.

Colorado Springs is laid out regularly, the streets munning east and west and north and south; the greatest extension is from nortl to south.

Generally speaking, it is inadvisable to have the astronomical station near the railroad-track; but in this ease the trains ran only during the day, and the observations were never affected by the vibrations of the ground. 


\section{Metionological Condtions.}

The meteorological observations made at the station show great ehanges in temperature during the day. I lave been told by several old residents that they never experienced a summer similar to that of 1873 . 'T'ie rainy period of the summer' is looked for about the Ist of July, to last only a few days. This year it was noted that from July 28 th to August gth there was no day without rain, accompanied by thunder and lightning. The prevailing winds were from the northeast or southeast, commencing at 10 or 11 o'clock a. m., and increasing in foree until 2 or 3 o'clock p. m. Then clouds came up from the soutliwest or west, bringing much rain, thunder, and lightning. It was generally elear again by 12 p. m.; but I found the air so very undulating, and the stars on that account so faint, that I was sometimes obliged to suspend the observations. It is probable that the temperature of the higher regions of the air was affected by the vicinity of the monntains, and after a rain changed very rapidly, while the lower strata remained under the same conditions.

The following table shows the general direction of the wind at 7 a. m., 2 p. m., and 9 p. m., giving the mean or prevailing direction of the wind for three hours before and three hours after the given time; also the estimated force of wind for the same time. The last column gives the general appearance of the sky, and needs no further explanation. It shows under what particularly unfavorable circnmstances the observations were made:

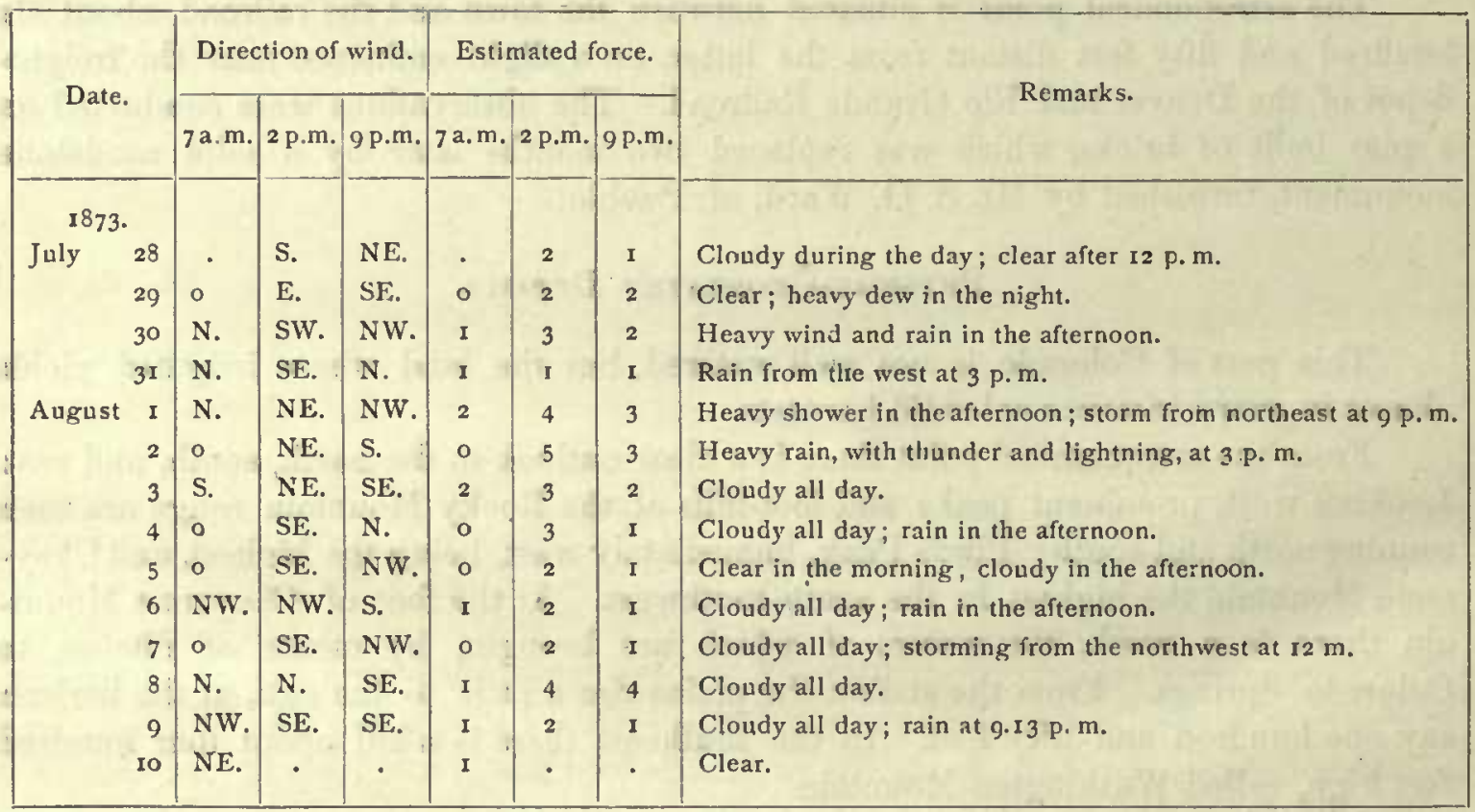

\section{Description of Observatory at Colorado Springs, Colorado Teritory.}

As soon as the monument was built, a solid framework, 8 by ro feet, was constructed, and a large wall-tent put over it. There was an opening in the tent for the meridian-line; this was closed, when necessary, by a fly. During heavy winds the 
tent was in rlanger of being blown away, and it was found necessary to nail the fly to the framework, while the tent itself was fastened to the stakes by strong iron wires. The entrance to the tent was from the west side, and was closerl by ropes. In the northwest corner of the observatory a large box was used for a table. On it the switchhoard and galvanic battery were placed; the chronometer being also placed there during the observations. The connection from the switch-board to the Western Union Telegraph office was made by a line 600 feet in lengtl, supported by the framework of the tent and one telegraph-post 30 fect in height. A gromol-wire was used after switching in the Western Union office to complete the circuit. In the northeast corner of the tent the chronograph was placed mpon in solid and insulated framework. Wires for the connection of the chronometer and breaking-key were fastened to the tent-fimme. The levels were also set on an insulated post in the southeast corner of the tent. For chairs I nsed two small boxes, one on the north and the other on the sonth side of the monument. In arranging and constructing the observing-tent I was assisted by C. I). Gedney and Privates J. Meier and .J. Clancy, Battalion of Engineer's. They also took the meteorologieal observations. $\mathrm{Mr}$. (*. 'T'. Ellison, at that time in charge of the Western Union office, kindly assisted in sending the telegraphic signals.

\section{DEscription of Instrunients UsED.}

Observations were made by means of a combined transit-iustrument number 28 , made by Wiirdemann. Its focal length is three feet; radius of aperture, $22_{4}^{3}$ inches; diameter of pivots, I $\frac{1}{4}$ inches. The diagonal eye-piece nsed had a magnifying-power of 40 diameters. This instrument was provided with two finding-circles, $3 \frac{1}{2}$ inches in diameter, graruated to every twenty minutes, and reading to single minutes by means of the vernier. Another circle was affixed to the ruper part of the tube, divided also also to twenty minutes, and having in the center a level used in latitude-observations for determining the change in the inclination of the horizontal levolving-base. Seven wires were placed in the focus for time-observations, besides one horizontal wire for latitude-observations. The equatorial intervals of the wires from mean of wires, rlamp west, upper culmination, were:

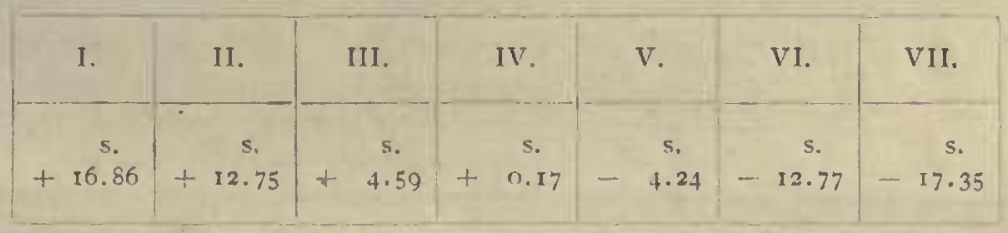

One revolution of the micrometer-screw moved the horizontal-wire $62^{\prime \prime}$. I 2 ; the the value of one division of the striding-level, which was used at every station, was $0^{\prime \prime} .75$; the value of one division of the zenith-telescope level was I". IO. The clironograpl used was similar to that used at the United States Naval Observatory invented by Professor. Willian Harkness; the barrel being 8 inches in diameter and 24 inclies long, and makes one revolution a minute. The chronograph worked very well when it was cleaned before commencing operations at a new station. It had but a single pen, which recorded clock-signals and those made by the observer. 
For time-observations and exchange, sidereal chronometer No. 1491, Negus, was always used. The galvanic connections were made by means of a switch-board, the connections of which are given in the following diagram:

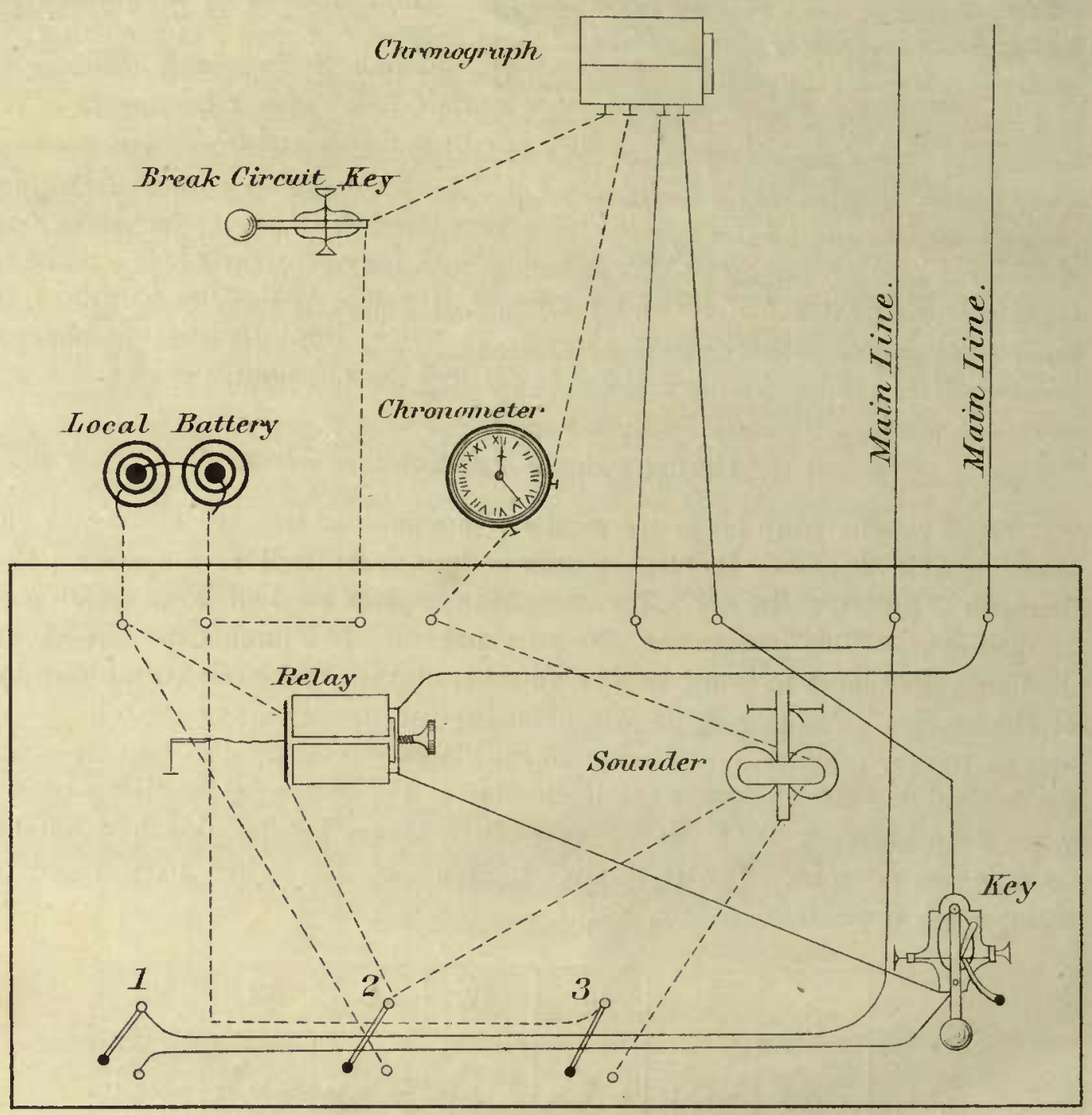

SwITCH x.-Closed when receiving from conuceted station; Nos. 2 and 3 open. Swıтcu 2.-Closed when sending to connected station; Nos. I and 3 open. SwITCH 3.-Local; throws sonnder into local circuit. 
Ponts with which Connections were Made, \&c.

Connection was made with Salt Lake City on the nights of July 29th and 3oth, and August 2d, 5th, and 6th. Observations for time were made at Colorado Springs on the nights of July 28th, 29th, 3oth, and 3 Ist, and August 2d, 4th, 5th, and 6th; at Salt Lake on the nights of July 28th, 29th, 3oth, and 3 Ist, and August Ist, 2d, 5th, and 6th.

The reductions of time-observations for Colorado Springs were made in the field by the astronomer, and also those made at Salt Lake after returning from the field. He also made a new reading of the signals sent and received from both stations.

The telegraph-line between Colorado Springs and Salt Lake is 763 miles long, and divided into four circuits. The signals are transmitted from one circuit to another by means of automatic repeaters: one placed at Denver, Colorado Territory; one at Cheyenne, Wyoming Territory; and one at Corinue, Utah: the length of the line being from Colorado Springs to Denver, 75 miles; from Denver to Cheyenne, ro6 miles; from Cheyenne to Corinne, 537 miles; and from Corinne to Salt Lake, 45 miles; using at every station sixty-five Grove cells.

The use of the wires was always freely tendered by the Western Union Telegraph Company, although in many cases they were needed at the same time for the transaction of the regular business of the company.

It sometimes occurs when two lines of wires are fixed to the same poles that, during heavy storins, the wires are brought in contact by oscillation, which was overcome in this case by connecting the two wires at an intermediate station, Denver, Colorado, upon the suggestion of Mr. Woodward, the superintendent at that point. 
Tabulation of Stars used for Determination of Time at Colorado Springs, Colorado, and Salt Lake City, Utah.

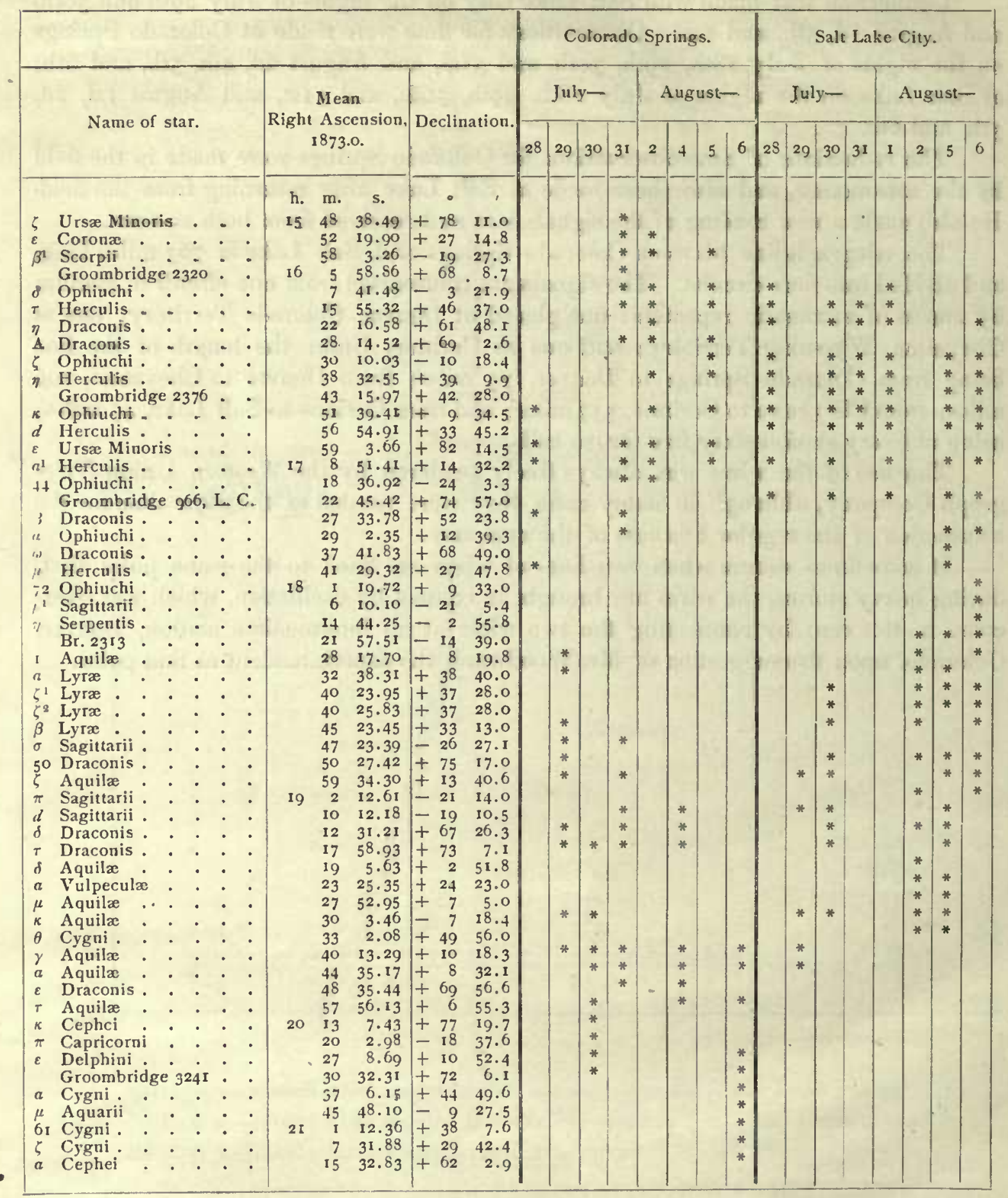


COLORADO SPRINGS, COLORADO TERRITORY, $f_{u l y} 28,1873$.

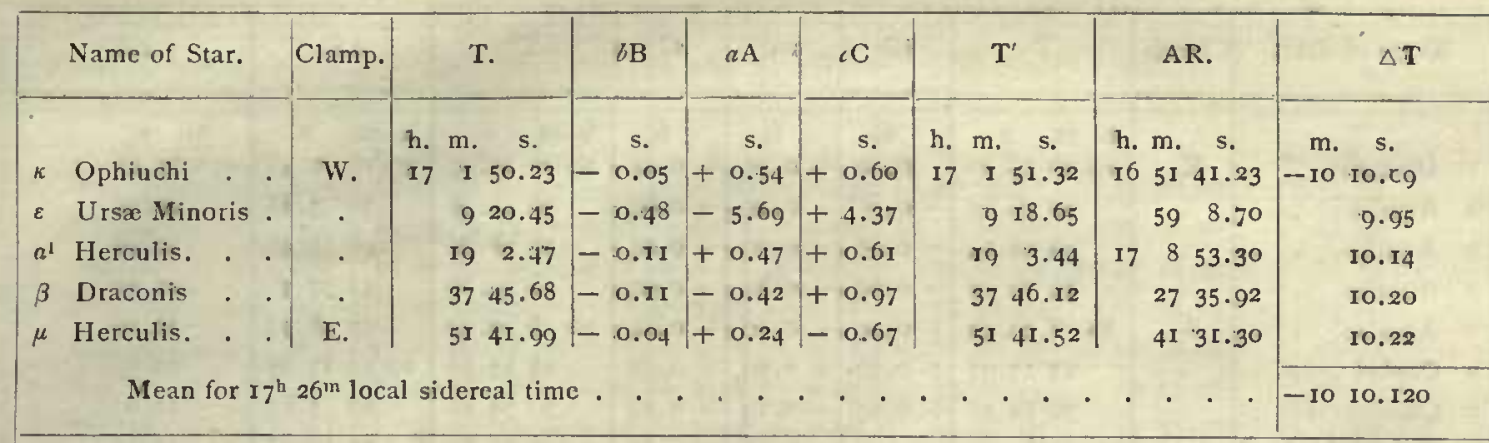

\section{Normal Equations.}

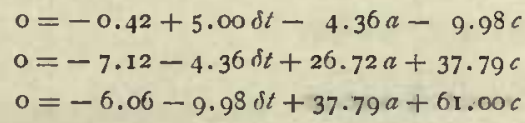

COLORADO SPRINGS, COLORADO TERRITORY, $\mathfrak{F}_{u l y} 29, \mathrm{I} 873$.

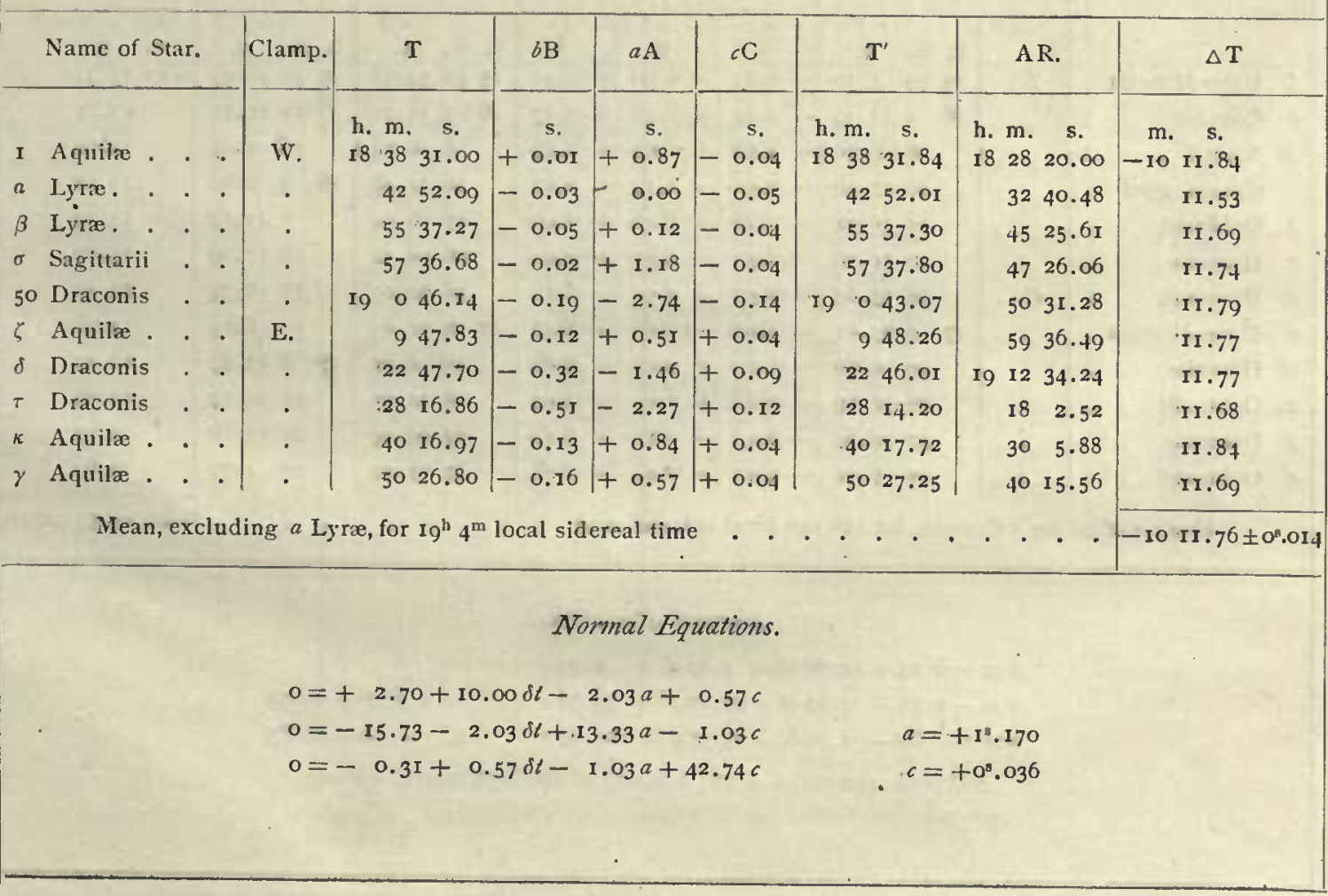


COLORADO SPRINGS, COLORADO TERRITORY, $f_{u l y} 3^{\circ}, 1873$.

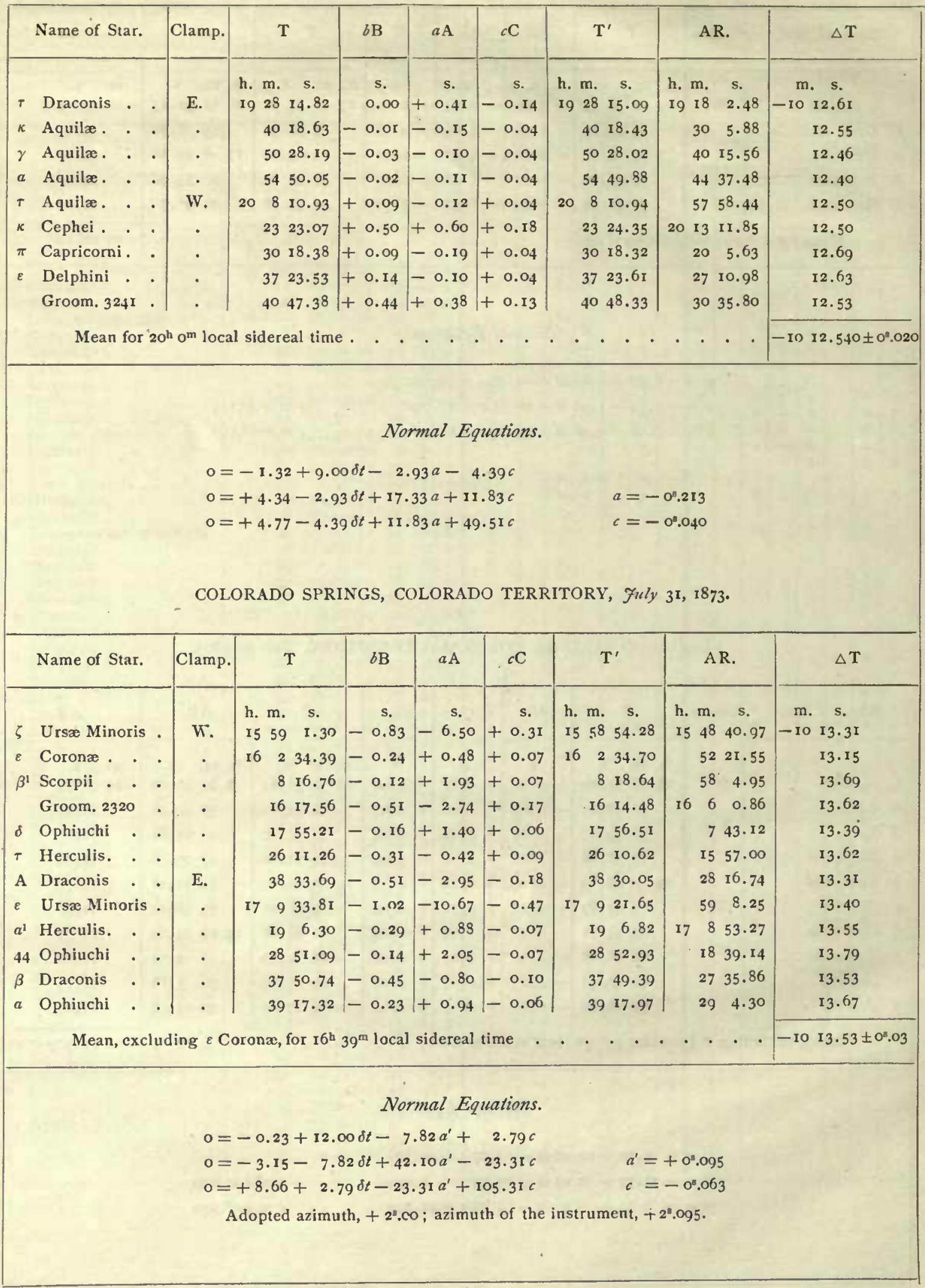


COLORADO SPRINGS, COLORADO TERRITORY, Fuly 31 I, 1873 .

\begin{tabular}{|c|c|c|c|c|c|c|c|c|c|}
\hline & Name of Star. & Clamp. & $\mathbf{T}$ & $b \mathrm{~B}$ & $a \mathbf{A}$ & cC & $\mathbf{T}^{\prime}$ & AR. & $\Delta \mathrm{T}$ \\
\hline$\sigma$ & Sagittarii . & E. & $\begin{array}{llc}\text { h. } & \text { m. } & \text { s. } \\
\text { I8 } & 57 & 37.8_{4}\end{array}$ & $\begin{array}{c}\text { s. } \\
- \text { o.II }\end{array}$ & $\begin{aligned} & s . \\
+ & 1.96\end{aligned}$ & $\begin{array}{c}\text { s. } \\
-0.07\end{array}$ & $\begin{array}{ccc}\text { h. } & \text { m. } & \text { s. } \\
\text { I8 } & 57 & 39.62\end{array}$ & 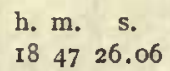 & $\begin{array}{cc}\text { m. } & \text { s. } \\
-10 & 13.56\end{array}$ \\
\hline & Aquilæ . & • & I9 949.73 & -0.20 & +0.86 & -0.07 & I9 950.32 & $\begin{array}{lll}59 & 36.48\end{array}$ & 13.84 \\
\hline a & Sagittarii . & . & 2027.04 & -0.12 & +1.75 & -0.07 & $2028.6 n$ & 19 10 14.75 & 13.85 \\
\hline$\delta$ & Draconis. & $\cdot$ & 2250.87 & -0.48 & -2.43 & -0.17 & $22 \quad 47 \cdot 79$ & 1234.19 & 13.60 \\
\hline$\tau$ & Draconis . & W. & 2820.22 & -0.46 & -3.79 & +0.22 & $28 \quad 16.19$ & $18 \quad 2.45$ & 13.74 \\
\hline$\gamma$ & Aquilx & - & 5028.22 & -0.09 & +0.95 & +0.07 & 5029.15 & $40 \quad 15.56$ & I3. 59 \\
\hline & Aquil $x$ & . & .5450 .10 & -0.05 & +0.99 & +0.06 & 5451.10 & $4437 \cdot 4^{8}$ & 13.62 \\
\hline$\varepsilon$ & Draconis . & . & $5^{8} \quad 55 \cdot 16$ & - o.10 & -2.94 & $1+0.19$ & $58 \quad 52.31$ & $48 \quad 38.70$ & $13.6 \mathrm{I}$ \\
\hline
\end{tabular}

\section{Normal Equations.}

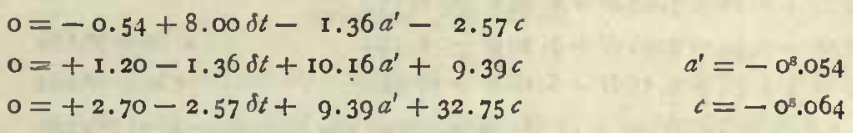

Adopted azimuth, $+2^{3} .00$; azimuth of the instrument, $+I^{8} .946$.

COLORADO SPRINGS, COLORADO TERRITORY, August 2, 1873.

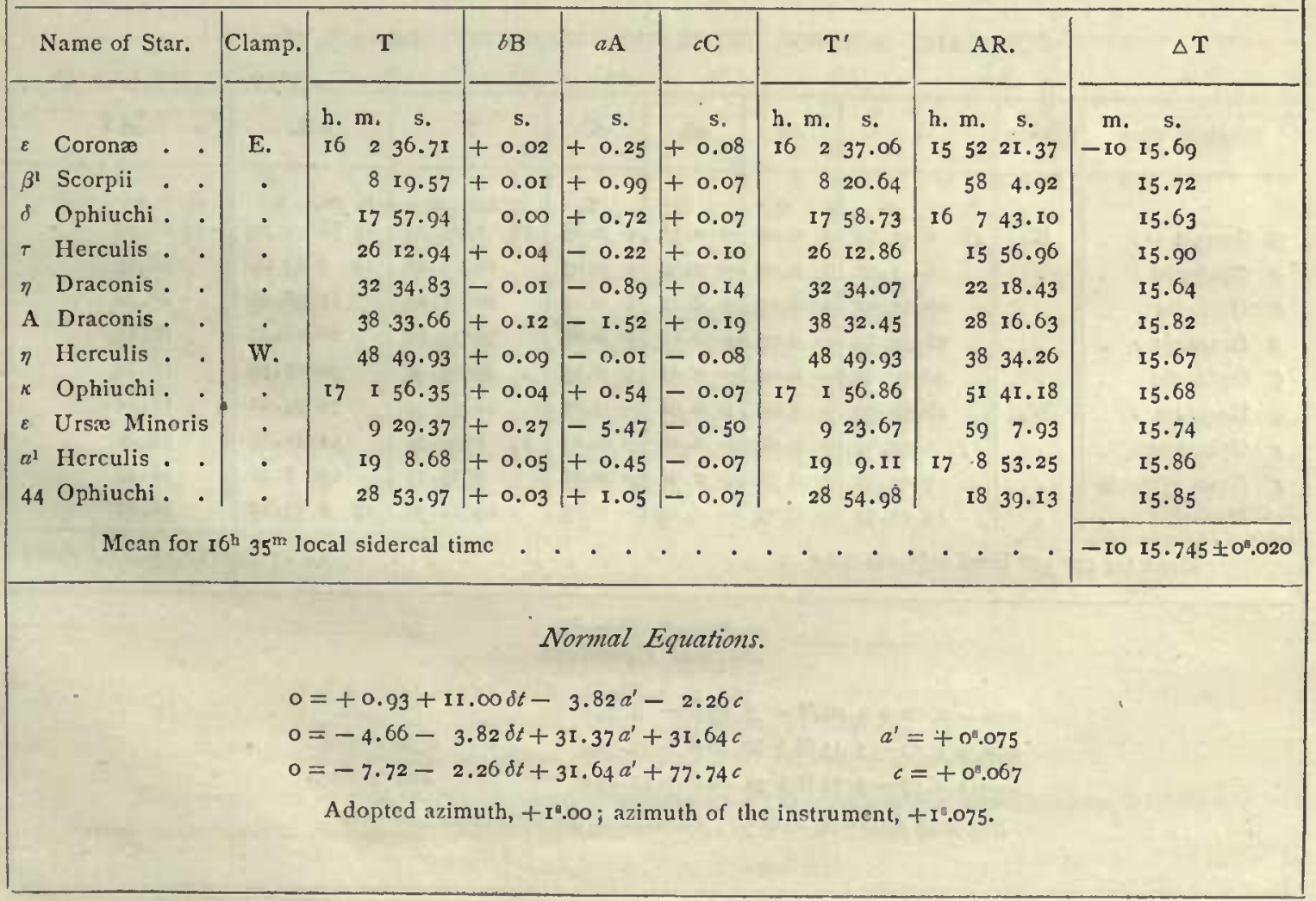


COLORADO SPRINGS, COLORADO ,TERRITORY, August 4, 1873.

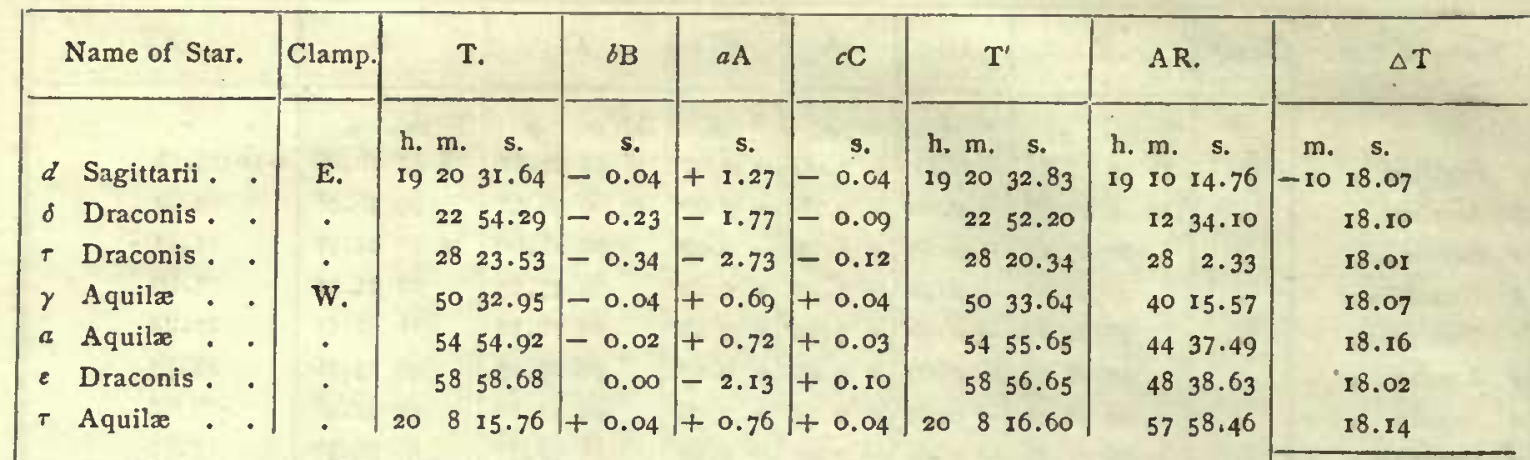

Mean for $19^{\mathrm{b}} 44^{\mathrm{m}}$ local sidercal time . . . . . . . . . . . . . . . : : $-1018.081 \pm 0.014$

Normal Equations.

$\begin{array}{ll}0=+0.16+7.00 \delta t-2.26 a^{\prime}+1.15 c & \\ 0=-0.93-2.26 \delta t+7.20 a^{\prime}-6.16 c & a^{\prime}=+0.110 \\ 0=+1.58+1.15 \delta t-6.16 a^{\prime}+31.46 c & c=-0.034\end{array}$

Adopted azimuth, $+\mathrm{I}^{\mathrm{B}} \cdot 30$; azimuth of the instrument, $+\mathrm{I}^{\mathrm{*}} \cdot 410$.

COLORADO SPRINGS, COLORADO TERRITORY, August 5, 1873.

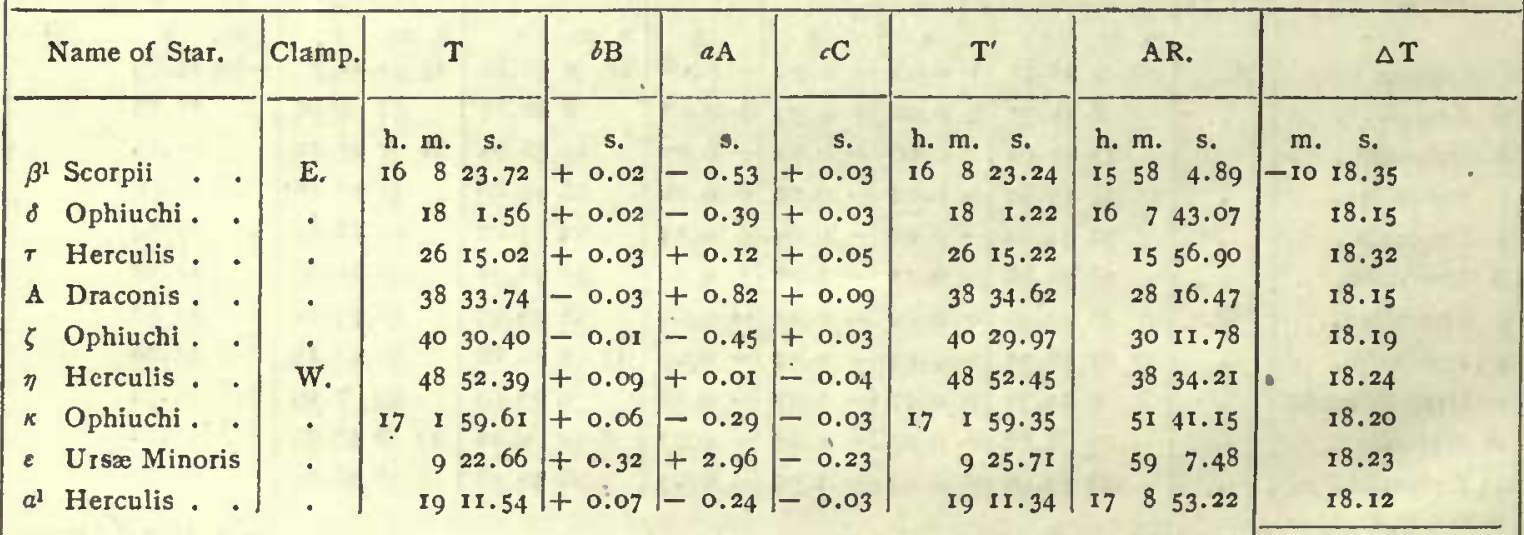

Mean for $16^{\text {b }} 33^{\text {m }}$ local sidereal time

$-1018.220 \pm 0.017$

\section{Normal Equations.}

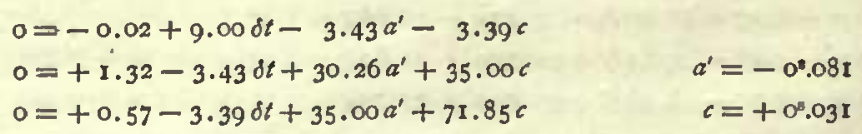

Adopted azimuth, -0.50 ; azimuth of the instrument, -0.581 . 
COLORADO SPRINGS, COLORADO TERRITORY, August 6, 1873.

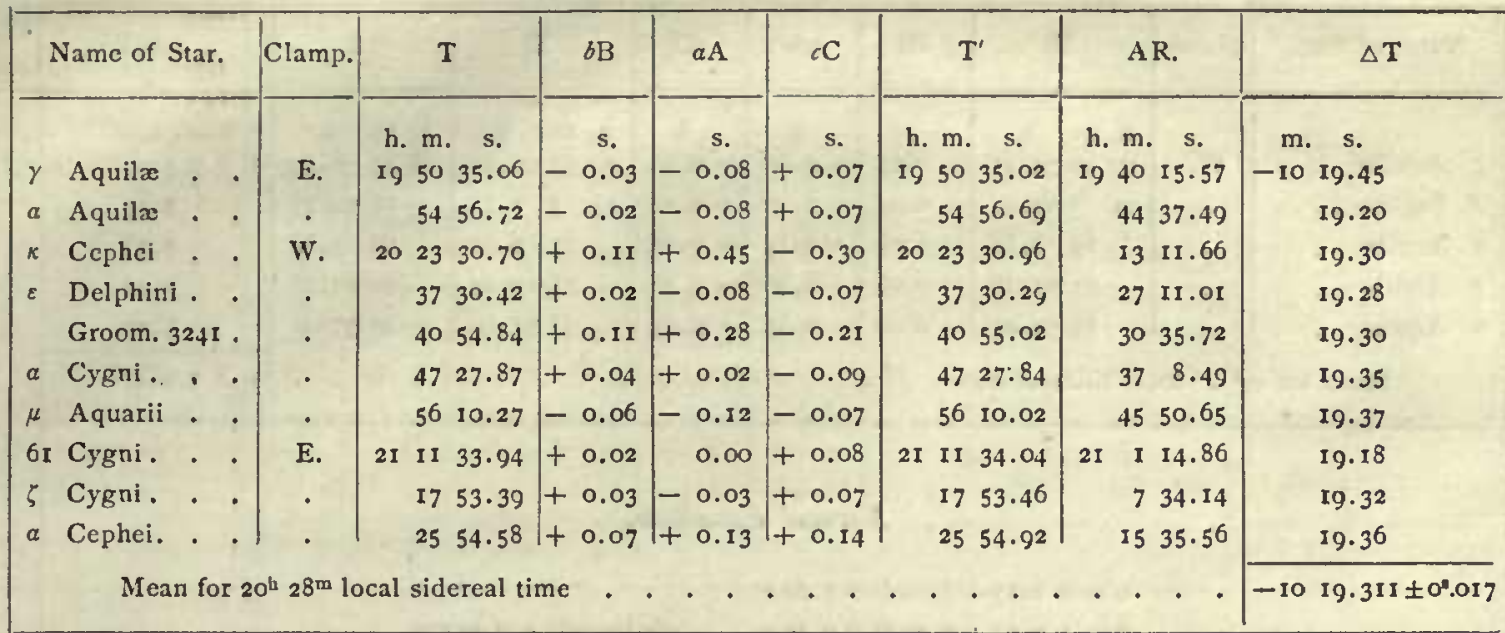

\section{Normal Equations.}

$$
\begin{array}{lll}
0=-2.07+10.00 \delta t-3.18 a-4.67 c & \\
0=+1.57-3.18 \delta t+13.31 a+17.08 c & a=-0^{8.159} \\
0=+0.59-4.67 \delta t+17.08 a+44.96 c & c=+0^{8} .067
\end{array}
$$

SALT LAKE CITY, UTAH TERRITORY, fuly 28,1873 .

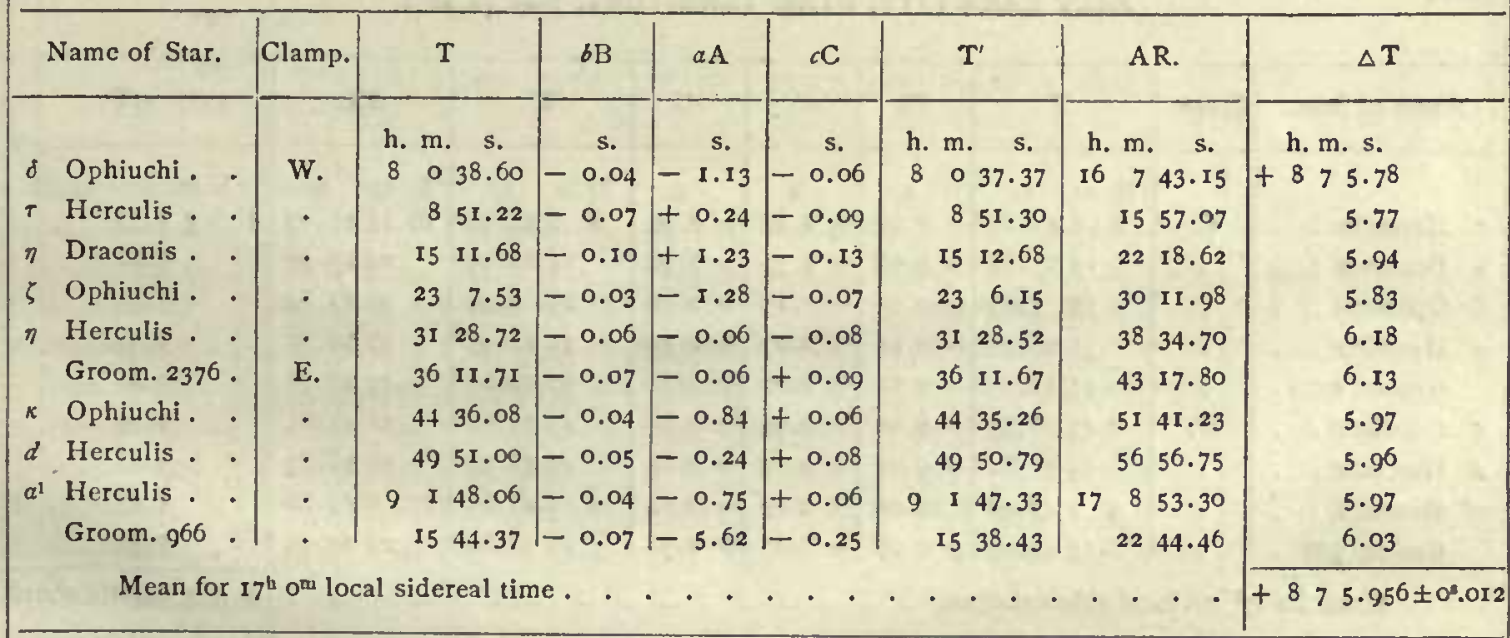

\section{Normal Equations.}

$$
\begin{array}{ll}
0=+0.15+11.00 \delta t+6.08 a-5.12 c & \delta t=-0^{9} .938 \\
0=-19.16+6.08 \delta t+14.92 a-11.02 c & a=+10.619 \\
0=+15.06-5.12 \delta t-11.02 a+31.57 c & c=-0.064
\end{array}
$$

These equations werc used when $v$ Serpentis was observed; the observation is excluded for the final result on account of the doubtful position of the star. 
SALT LAKE CITY, UTAH TERRITORY, Fuly 29, 1873 .

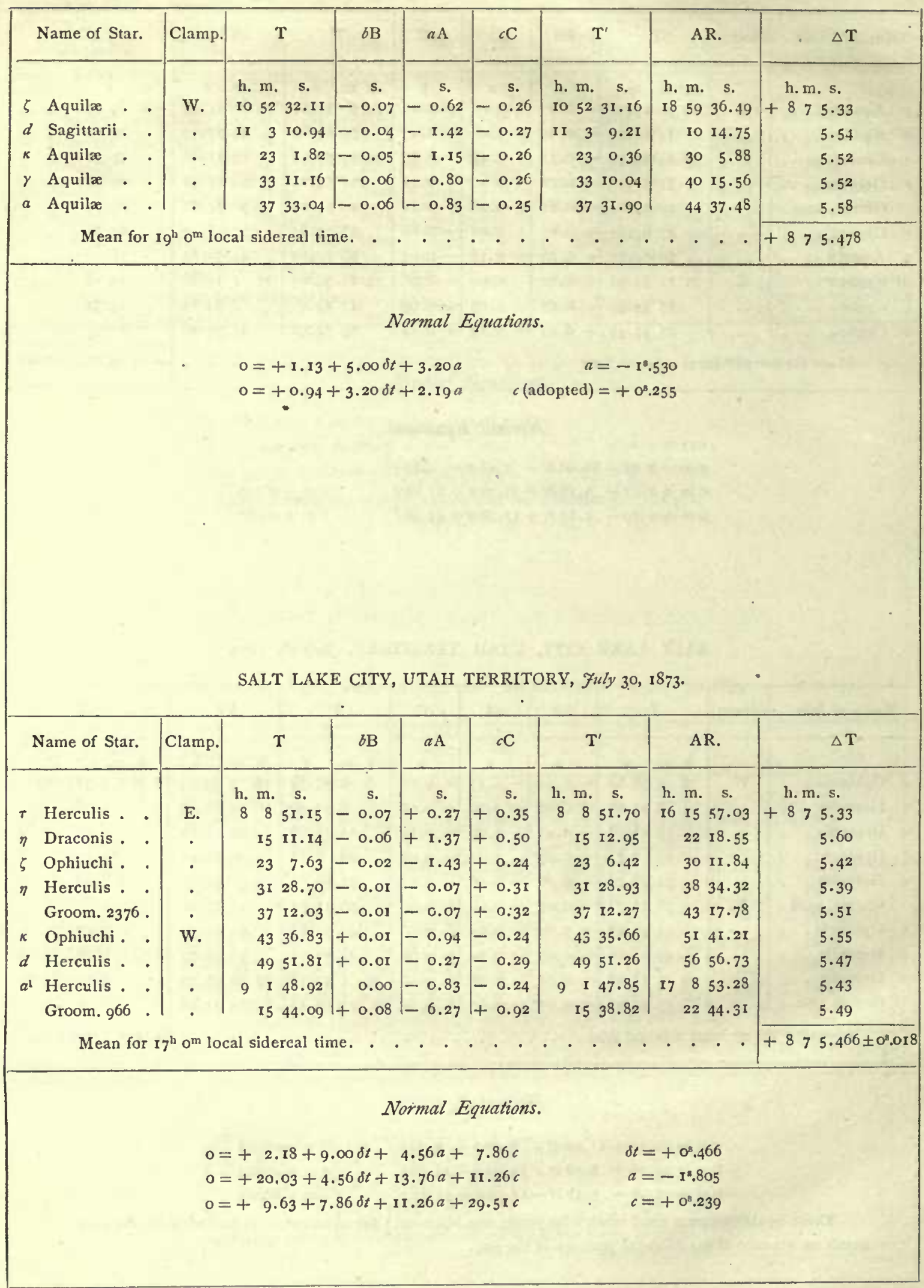


SALT LAKE CITY, UTAH TERRITORY, $f_{u l y} 3^{\circ}$, 1873.

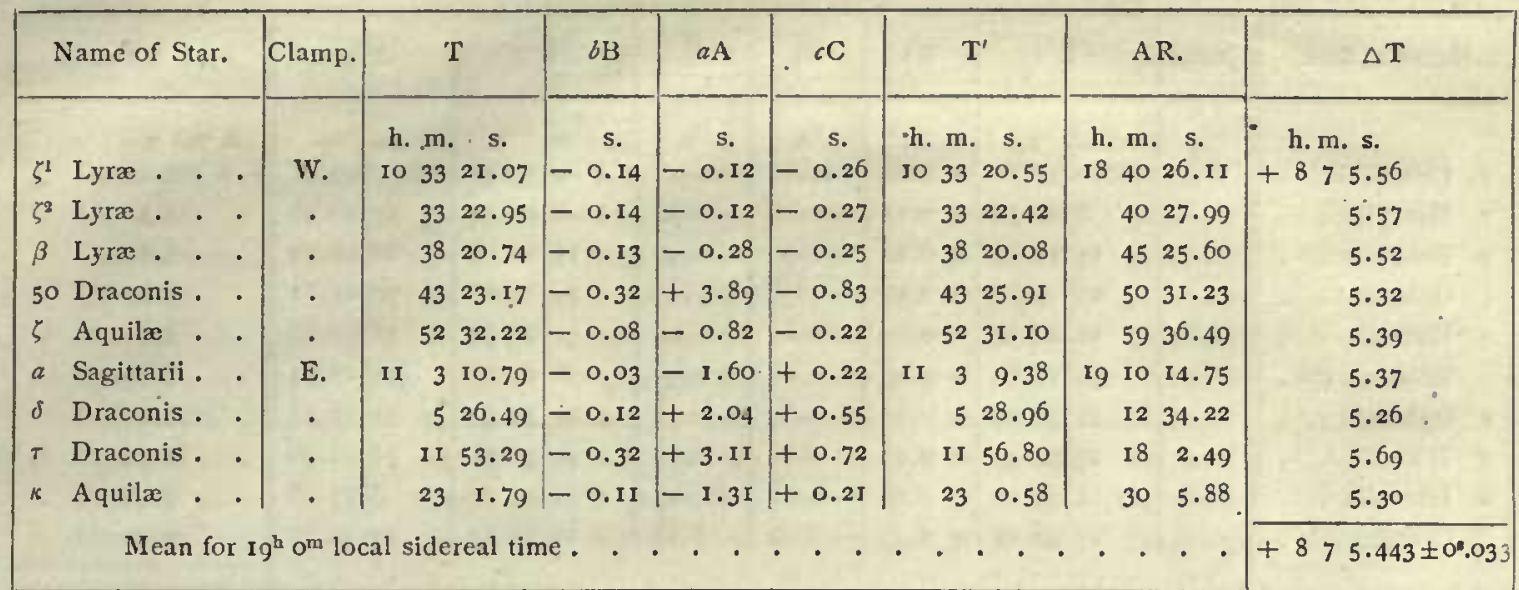

\section{Normal Equations.}

$$
\begin{array}{ll}
0=-8.65+9.00 \delta t-2.80 a-0.57 c & \delta t=+0^{8.443} \\
0=+21.01-2.80 \delta t+11.39 a+0.30 c & a=-1.744 \\
0=-8.05-0.57 \delta t+0.30 a+41.98 c & c=+0^{8} .210
\end{array}
$$

SALT LAKE CITY, UTAH TERRITORY, $\mathfrak{f u l y}^{3}$ 3, 1873.

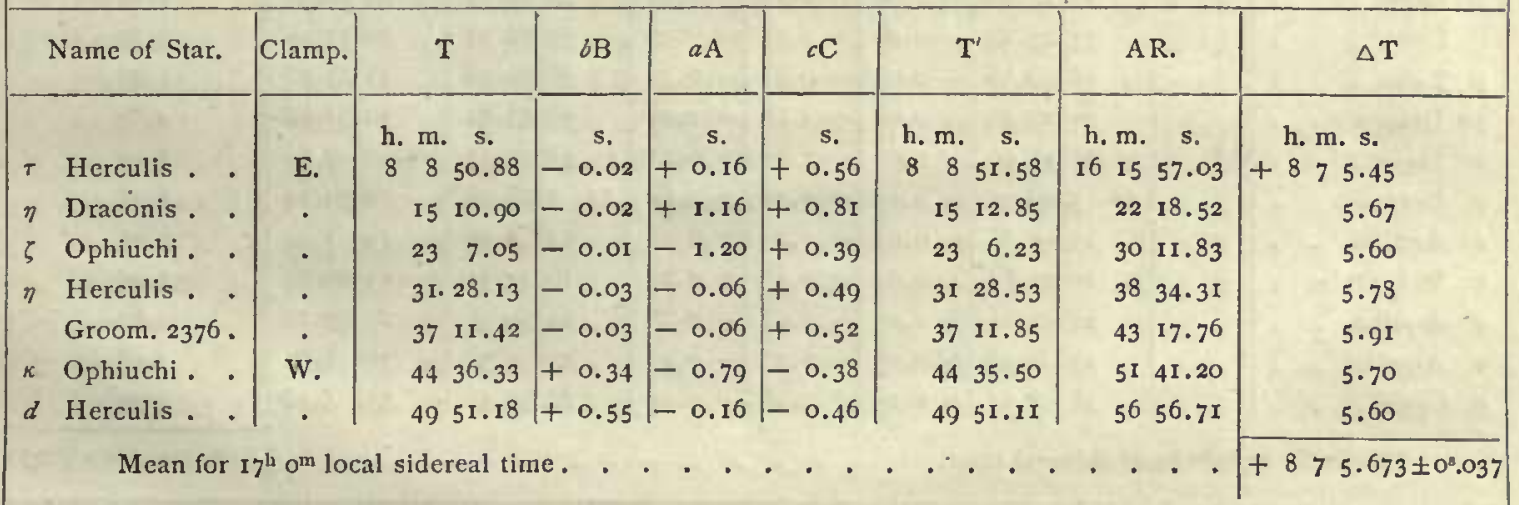

\section{Normal Equations.}

$$
\begin{array}{ll}
0=-5.69+7.00 \delta t+0.63 a+5.04 c & \delta t=+0^{8} .67 \\
0=+2.50+0.63 \delta t+1.51 a-1.63 c & a=-I^{2} .520 \\
0=-11.01+5.04 \delta t-1.63 a+13.63 c & c=+0^{8} .38 \mathrm{I}
\end{array}
$$


SALT LAKE CITY, UTAH TERRITORY, August I, 1873.

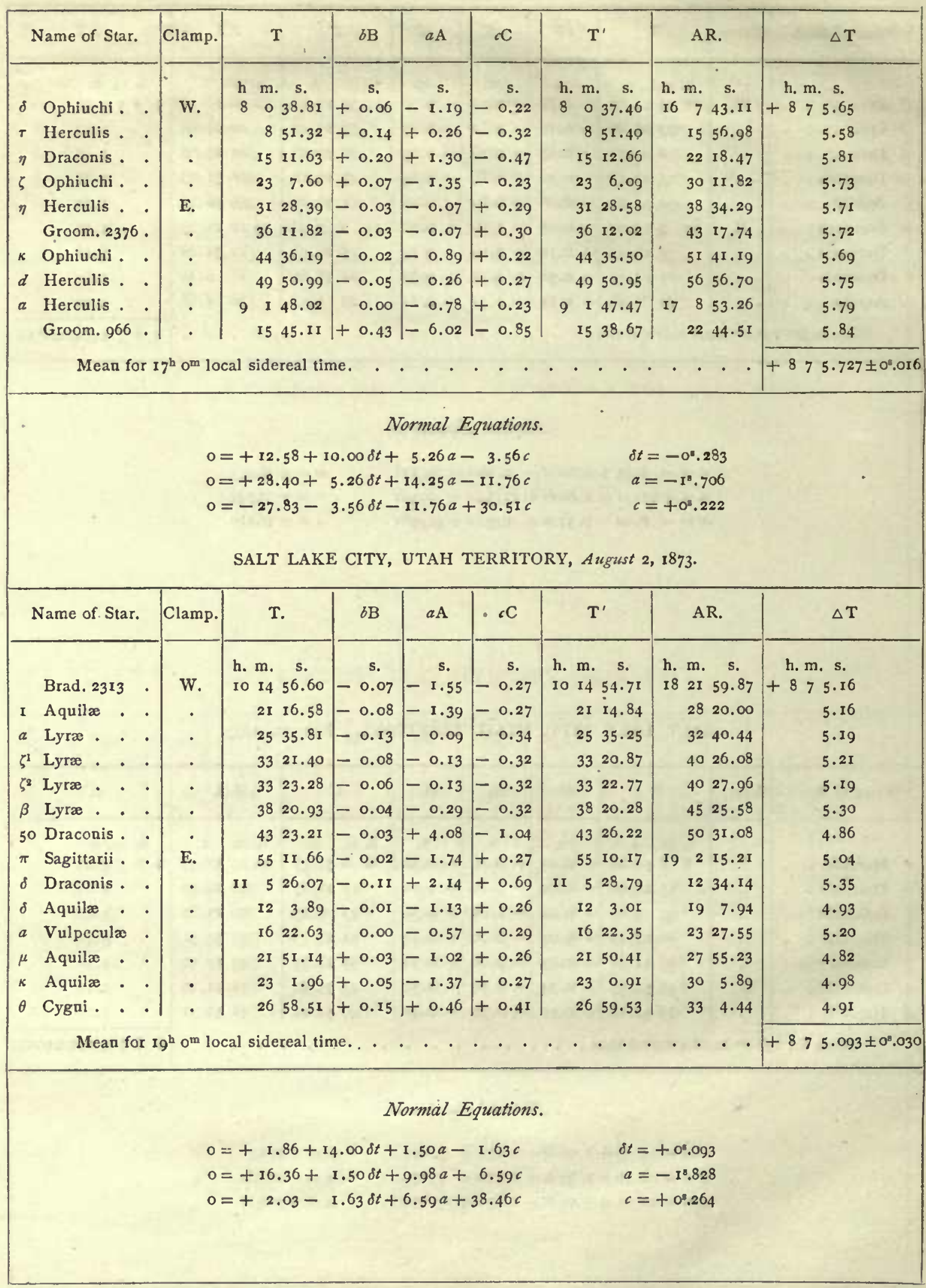


SALT LAKE CITY, UTAH TERRITORY, Augzst 5, I873.

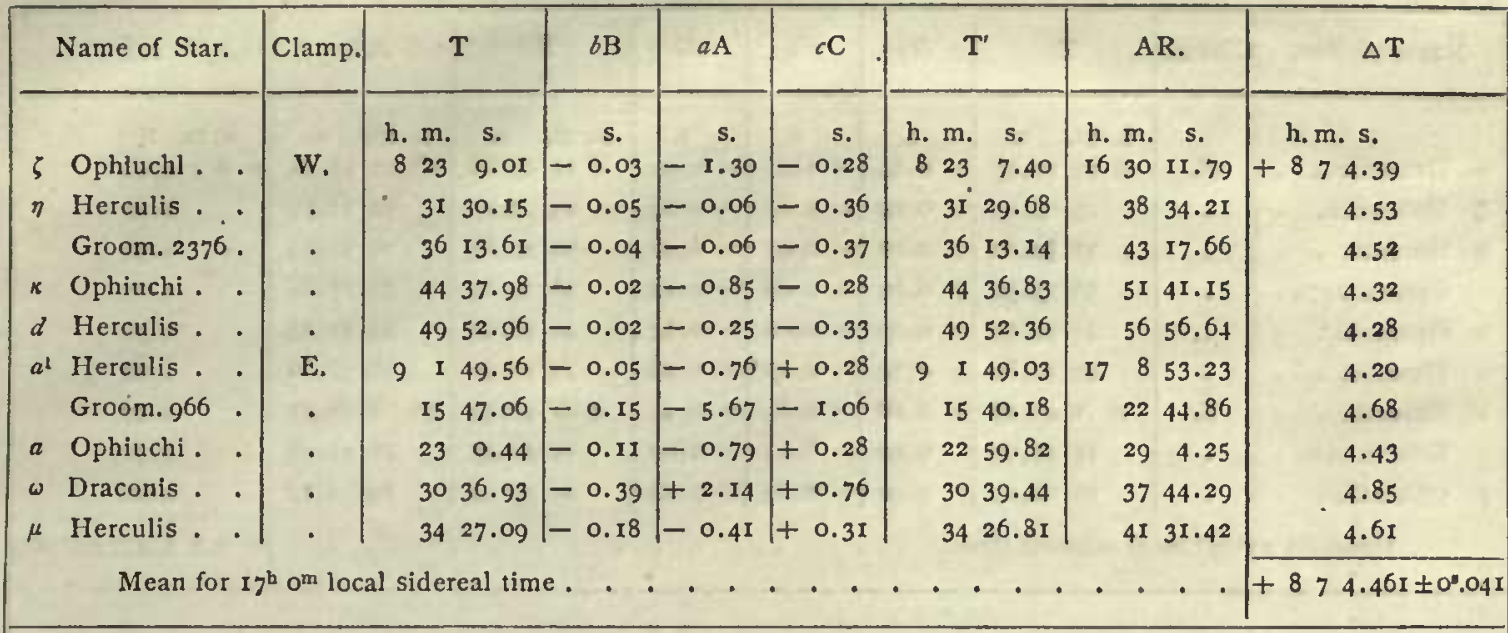

Normal Equations.

$$
\begin{array}{ll}
0=+4.45+10.00 \delta t+4.90 a-3.77 c & \delta t=+0^{8} .461 \\
0=+27.37+4.90 \delta t+15.14 a-17.34 c & a=-1^{8} .643 \\
0=-35.79-3.77 \delta t-17.34 a+32.90 c & c=+0^{8.275}
\end{array}
$$

SALT LAKE CITY, UTAH TERRITORY, August 5, 1873 .

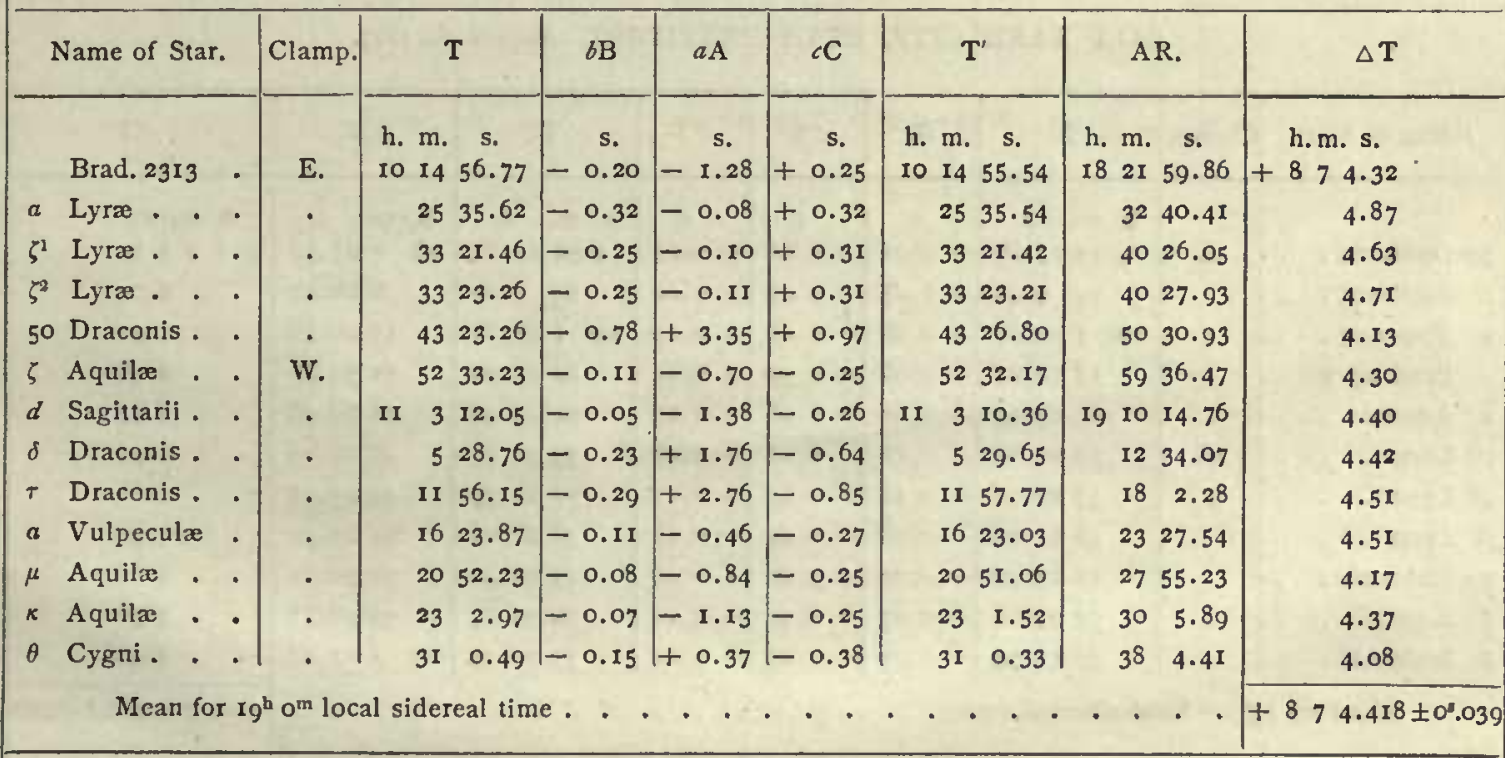

Normal Equations.

$$
\begin{array}{ll}
0=-6.50+13.00 \delta t-1.44 a-4.04 c & \delta t=+0^{8} .418 \\
0=+19.41-1.44 \delta t+12.55 a-1.04 c & a=-1^{8} .503 \\
0=-11.79-4.04 \delta t-1.04 a+47.87 c & c=+0^{8} .247
\end{array}
$$


SALT LAKE CITY, UTAH TERRITORY, August 6, 1873.

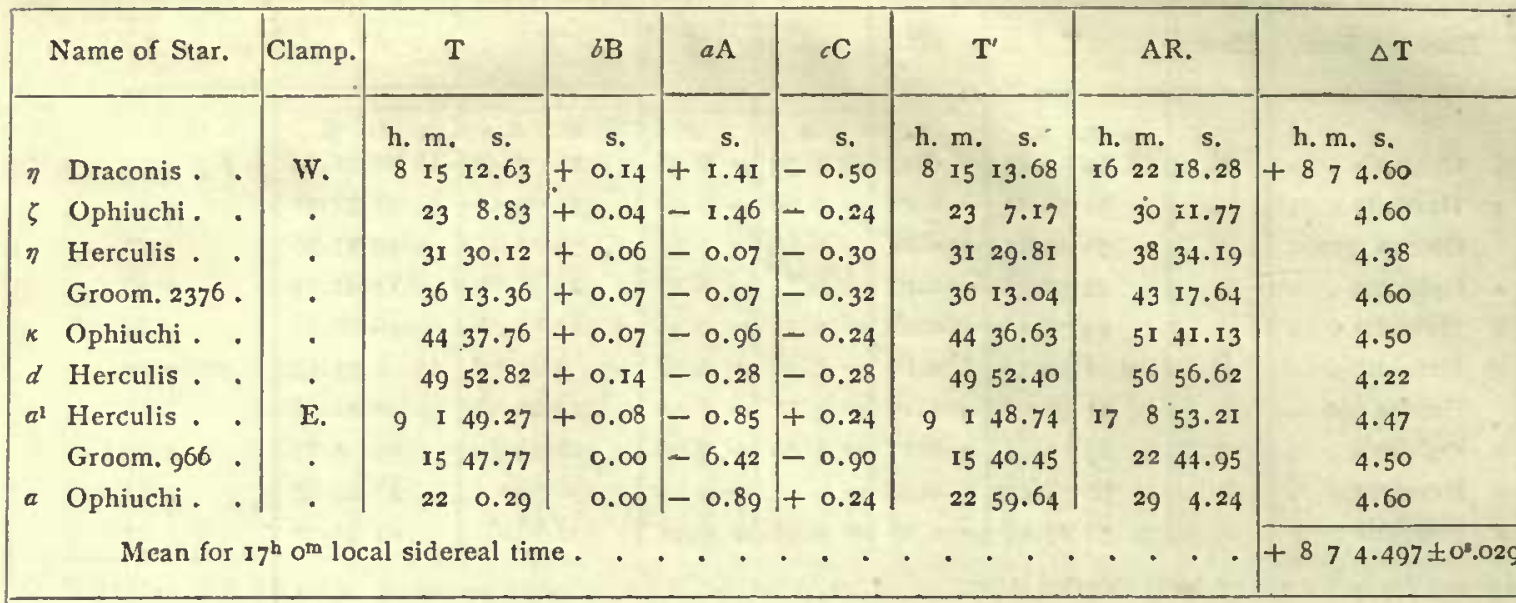

\section{Normal Equations.}

$$
\begin{array}{ll}
0=+3.82+9.00 \delta t+5.19 a-9.79 c & \delta t=+0.897 \\
0=+24.10+5.19 \delta t+13.97 a-12.41 c & a=-1.850 \\
0=-20.84-9.79 \delta t-12.41 a+28.44 c & c=+0.234
\end{array}
$$

SALT LAKE CITY, UTAH TERRITORY, August 6, 1873.

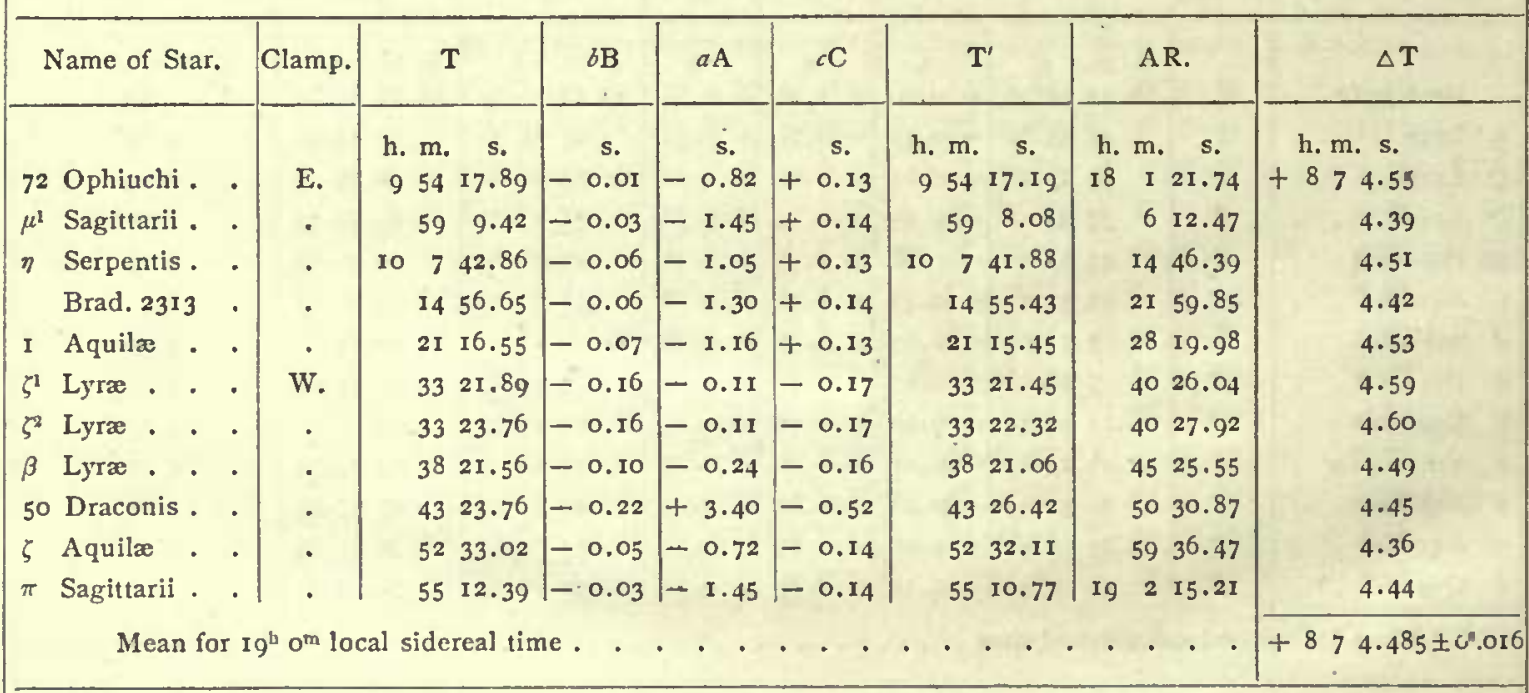

Normal Equations.

$$
\begin{array}{ll}
0=+0.31+11.00 \delta t+3.27 a-4.63 c & \delta t=+0^{8} .485 \\
0=+10.84+3.27 \delta t+9.08 a+10.82 c & a=-I^{11.525} \\
0=+15.07-4.63 \delta t+10.82 a+27.60 c & c=+0^{11.133}
\end{array}
$$


Taking the mean of the determinations of the corrections of the chronometer, the following table shows the corrections and the adopted rates of the chronometers used at Colorado Springs and Salt Lake City:

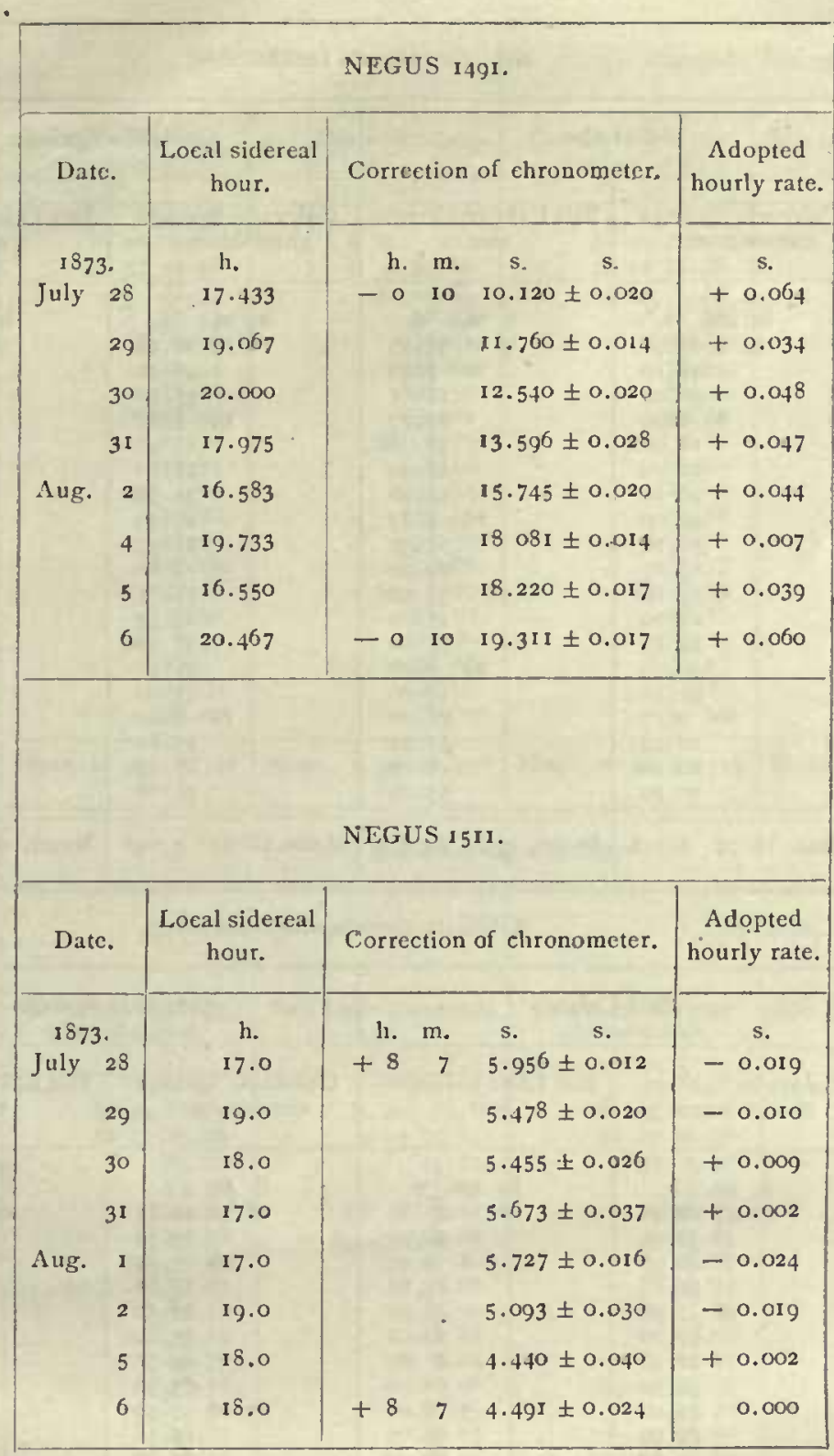

9 
Signals for Determination of Longitude between Salt Lake City, Utuh, and Colorado Springs, Coloralo Territory.

\begin{tabular}{|c|c|c|c|c|}
\hline \multirow{3}{*}{ Date. } & \multicolumn{3}{|c|}{ Arbitrary signals sent from- } & \\
\hline & \multicolumn{2}{|c|}{ Salt Lake. } & \multicolumn{2}{|c|}{ Colorado Springs. } \\
\hline & $\begin{array}{l}\text { Colorado Springs } \\
\text { chronometer. }\end{array}$ & $\begin{array}{l}\text { Salt Lake chronom- } \\
\text { eter. }\end{array}$ & $\begin{array}{l}\text { Colorado Springs } \\
\text { chronometer. }\end{array}$ & $\begin{array}{l}\text { Salt Lake chronom- } \\
\text { eter. }\end{array}$ \\
\hline \multirow[t]{2}{*}{$\begin{array}{l}\text { I } 873 . \\
\text { July } 29\end{array}$} & $\begin{array}{rr}\text { h. m. } & s . \\
\text { I8 } 7 & 29.91 \\
& 40.10 \\
& 50.00 \\
8 & 0.02 \\
10.00 \\
20.00 \\
30.10 \\
40.00 \\
50.16 \\
59.87 \\
910.16 \\
20.19 \\
30.19 \\
40.20 \\
50.18 \\
10 \quad 0.17 \\
10.40 \\
20.20 \\
30.20\end{array}$ & \begin{tabular}{rr} 
h. m. & \multicolumn{1}{c}{ s. } \\
$92 \mathrm{I}$ & 54.77 \\
22 & 4.90 \\
& 14.85 \\
24.87 \\
34.90 \\
44.90 \\
54.96 \\
$23 \quad 4.87$ \\
14.97 \\
24.70 \\
34.99 \\
45.00 \\
55.00 \\
5.00 \\
15.00 \\
25.00 \\
35.22 \\
45.03 \\
55.10
\end{tabular} & $\begin{array}{rr}\text { h. m. } & \text { s. } \\
\text { I8 I3 } & 30.52 \\
& 40.52 \\
& 55.10 \\
\text { I4 } & 5.62 \\
& 15.96 \\
& 25.53 \\
& 35.56 \\
45.43 \\
\\
55.57 \\
\text { I5 } 5.60 \\
15.75 \\
25.55 \\
35.48 \\
46.10 \\
55.00 \\
76 \\
7.00 \\
\text { I5.60 } \\
25.88 \\
35.10\end{array}$ & $\begin{array}{rr}\text { h. m. s. } \\
927 & 55.60 \\
28 & 5.60 \\
20.15 \\
30.70 \\
41.03 \\
50.60 \\
29 \quad 0.62 \\
10.50 \\
20.65 \\
30.70 \\
40.85 \\
50.63 \\
30.53 \\
0.18 \\
1 \mathrm{~J} \\
20.09 \\
32.10 \\
40.70 \\
50.98 \\
31 & 0.20\end{array}$ \\
\hline & Mean.18,90.108 & Mean. 92324.949 & Mean. IS I5 5.098 & Mean. 92930.169 \\
\hline \multirow{3}{*}{ Date. } & \multicolumn{3}{|c|}{ Arbitrary signals sent from- } & \\
\hline & \multicolumn{2}{|c|}{ Salt Lake. } & \multicolumn{2}{|c|}{ Colorado Springs. } \\
\hline & $\begin{array}{l}\text { Colorado Springs } \\
\text { chronometer. }\end{array}$ & $\begin{array}{l}\text { Salt Lake chronom- } \\
\text { eter. }\end{array}$ & $\begin{array}{l}\text { Colorado Springs } \\
\text { chronometer. }\end{array}$ & $\begin{array}{c}\text { Salt Lake chronom- } \\
\text { eter. }\end{array}$ \\
\hline \multirow[t]{2}{*}{$\begin{array}{l}\text { I } 873 . \\
\text { July } 30\end{array}$} & 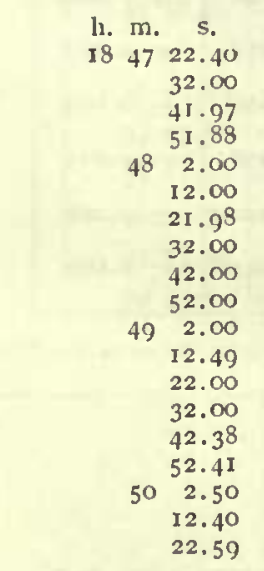 & $\begin{array}{cc}\text { h. m. } & \text { s. } \\
\text { Io I } & 46.37 \\
56.00 \\
2 \quad 5.97 \\
15.89 \\
26.00 \\
36.02 \\
46.00 \\
56.00 \\
3 \quad 6.00 \\
16.00 \\
26.00 \\
36.47 \\
46.10 \\
56.00 \\
46.38 \\
16.43 \\
26.48 \\
36.41 \\
46.60\end{array}$ & 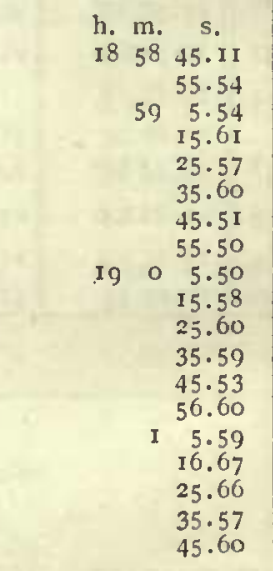 & $\begin{array}{rr}\text { h. m. } & \text { s. } \\
\text { I0 I3 } & 9.48 \\
\text { I } 9.90 \\
29.90 \\
40.00 \\
49.92 \\
59.94 \\
9.90 \\
\text { I4 } 19.88 \\
29.86 \\
39.94 \\
49.93 \\
59.94 \\
9.90 \\
20.93 \\
29.90 \\
41.00 \\
49.99 \\
59.90 \\
9.95\end{array}$ \\
\hline & Mean. Is $4^{3} \quad 52.158$ & Mean.jo 316.164 & Mean. Ig o 15.656 & Nean. Io 1440.008 \\
\hline
\end{tabular}


Signals for Determination of Longitude, dc.-Continued.

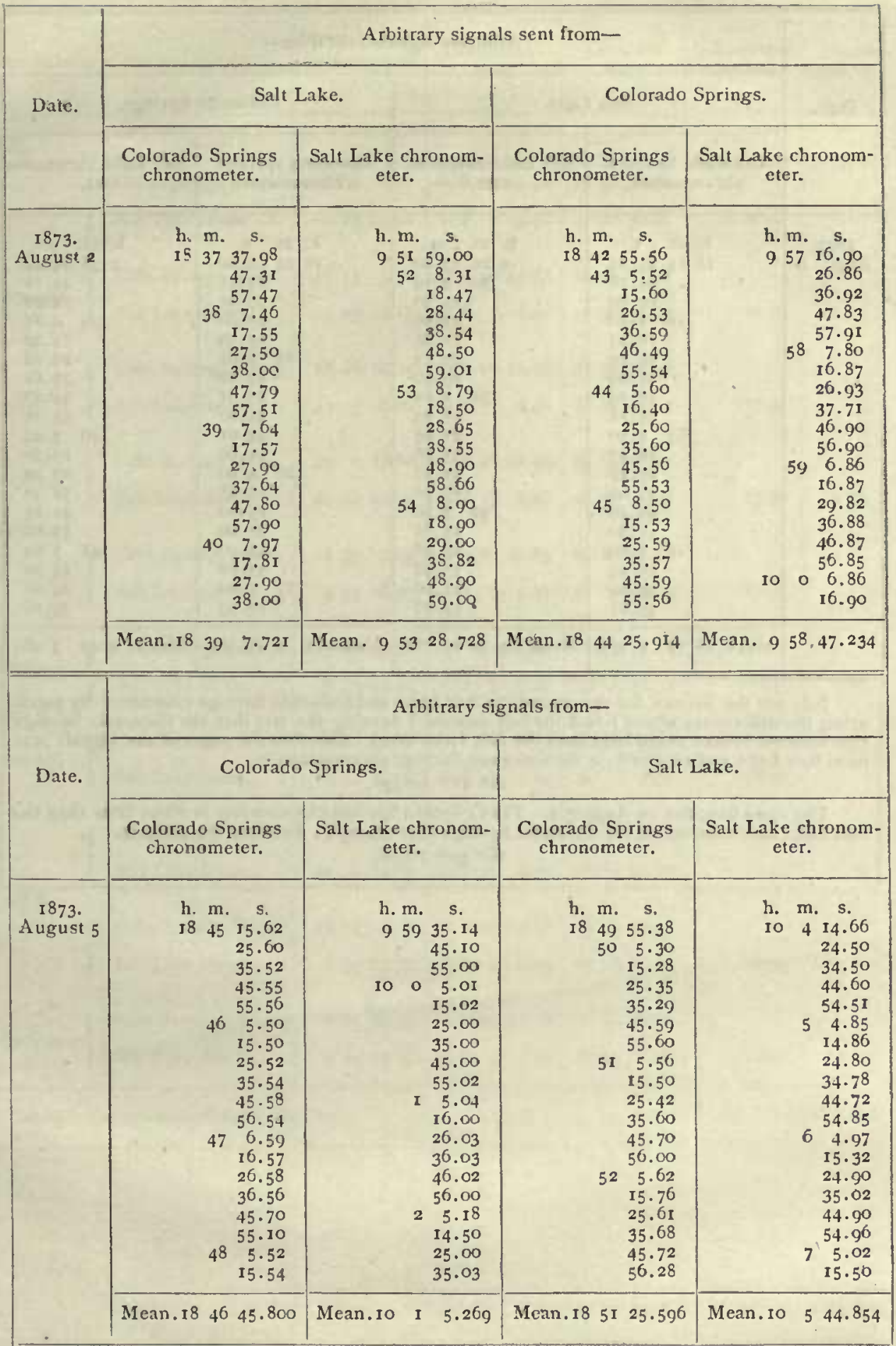


Signals for Determination of Longitude, dec.-Continued.

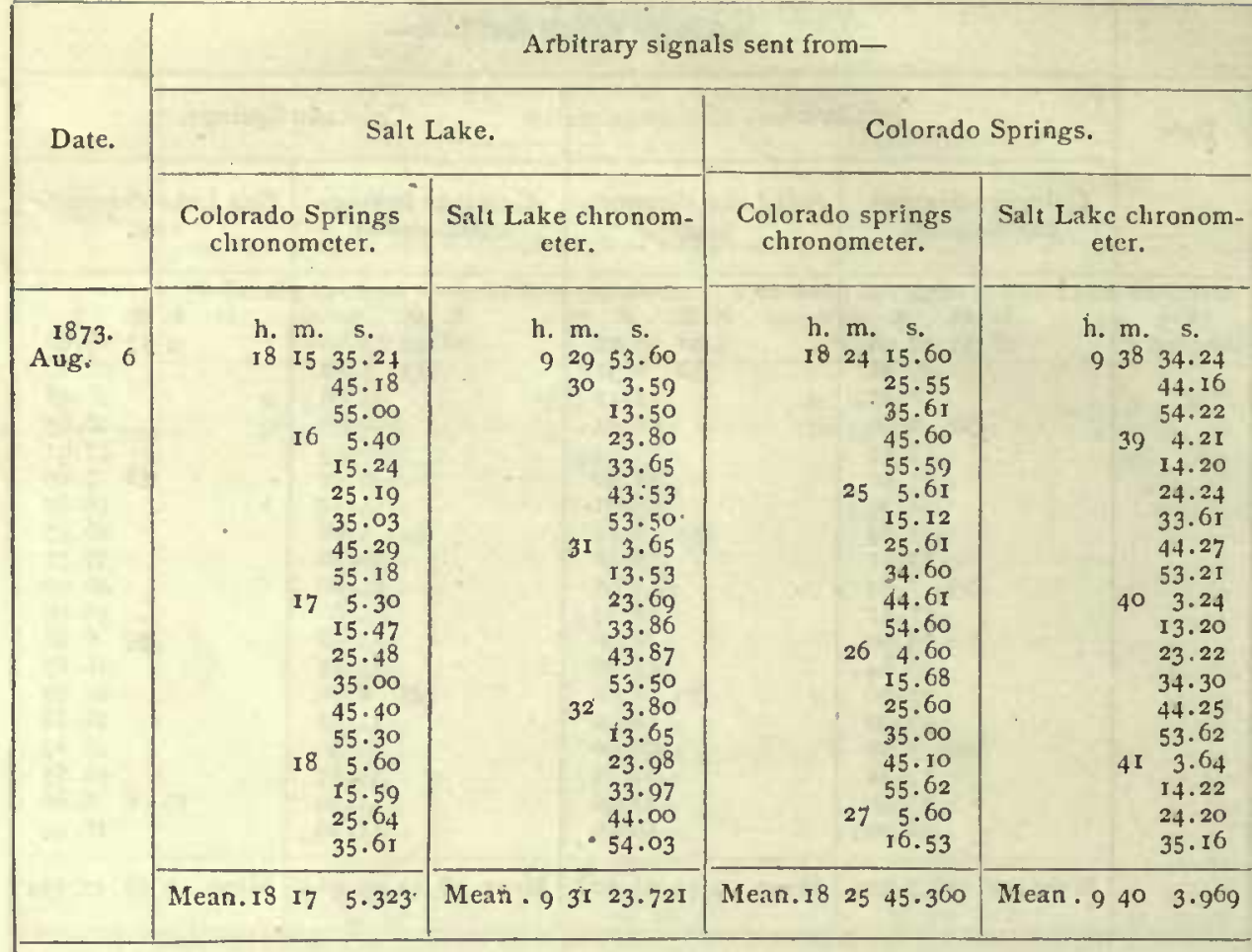

July 30 , the breaks for the seconds of Salt Lake and Colorado Springs coincide. By measuring the differences whcre I find the full minute, I develop the fact that the Colorado Springs chronometcr breaks $0^{*}$. I Io later than the Satt Lake break; thereforc the mean of the signals sent from Salt Lake and received on the Colorado Springs chronograph is,

$18^{\mathrm{h}} 4^{8^{\mathrm{m}}} 52^{\mathrm{m}} .04^{8}$.

The same happens on August 2. The Colorado Springs chronometer is $0^{*} .095$ later than the Salt Lake chronometer on the Colorado Springs chronograph; therefore the mcan is,

$$
18^{\mathrm{h}} 39^{\mathrm{m}} 7^{\mathrm{a}} .626 \text {. }
$$


Final Results for Longitude.

\begin{tabular}{|c|c|c|c|c|c|c|c|}
\hline Signals sent from- & Stations of record. & $\begin{array}{l}\text { Mean of sig- } \\
\text { nals sent and } \\
\text { received. }\end{array}$ & $\begin{array}{c}\text { Time-correc- } \\
\text { tion. }\end{array}$ & $\begin{array}{c}\text { Corrected } \\
\text { time. }\end{array}$ & $\begin{array}{l}\text { Difference of } \\
\text { longitude. }\end{array}$ & $\begin{array}{c}\text { Double } \\
\text { wave-time. }\end{array}$ & Means. \\
\hline $\begin{array}{c}1873 . \\
f_{u l y} 29 . \\
\text { Salt Lake... }\end{array}$ & $\begin{array}{l}\text { Colo. Springs chron. } \\
\text { Salt Lake chron. . - }\end{array}$ & $\begin{array}{ccc}\text { h. } & \text { m. } & \text { s. } \\
\text { I } 8 & 9 & 0.11 \\
& & \\
9 & 23 & 24.95\end{array}$ & $\begin{array}{r}\text { h. m. s. } \\
-0 \text { 10 11:72 } \\
+875.46\end{array}$ & $\begin{array}{lcc}\text { h. } & \text { m. } & \text { s. } \\
\text { I } 7 & 5^{8} & 48.39 \\
\text { I } & 30 & 30.41\end{array}$ & $\begin{array}{l}\text { h. m. s. } \\
\text { O } 28 \times 7.98\end{array}$ & s. & s. \\
\hline Colorado Springs - \{ & $\begin{array}{l}\text { Colo. Springs chron. } \\
\text { Salt Lake chron. . }\end{array}$ & $\begin{array}{lll}18 & 15 & 5.10 \\
9 & 29 & 30.17\end{array}$ & $\begin{array}{r}-01011.72 \\
+875.46\end{array}$ & $\begin{array}{l}18 \quad 453.3^{8} \\
17 \quad 36 \quad 35.63\end{array}$ & $17 \cdot 75$ & 0.23 & 17.865 \\
\hline $\begin{array}{c}\mathcal{F} u l y \text { 3o. } \\
\text { Salt Lake ... }\end{array}$ & $\begin{array}{l}\text { Colo. Springs chron. } \\
\text { Salt Lake chron. . }\end{array}$ & $\begin{array}{l}18 \quad 4^{8} 52.05 \\
\text { 10 } 316.16\end{array}$ & $\begin{array}{l}-0 \text { 10 } 12.48 \\
+875.46\end{array}$ & $\begin{array}{lll}\text { I8 } & 3^{8} & 39.57 \\
\text { I } & \text { 10 } & 21.62\end{array}$ & 17.95 & & \\
\hline Colorado Springs . \{ & $\begin{array}{l}\text { Colo. Springs chron. } \\
\text { Salt Lake chron. . }\end{array}$ & 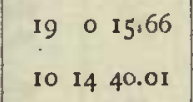 & $\begin{array}{r}-0 \text { 10 12.49 } \\
+875.46\end{array}$ & $\begin{array}{lll}\text { I8 } & 50 & 3.17 \\
\text { I8 } & 21 & 45.47\end{array}$ & 17.70 & 0.25 & 17.825 \\
\hline Salt Lake... & $\begin{array}{l}\text { Colo. Springs chron. } \\
\text { Salt Lake chron. . - }\end{array}$ & 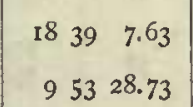 & $\begin{array}{l}-01015.83 \\
+875.11\end{array}$ & $\begin{array}{lll}18 & 28 & 51.80 \\
18 & 0 & 33.84\end{array}$ & 17.96 & & \\
\hline Colorado Springs - \{ & $\begin{array}{l}\text { Colo. Springs chron. } \\
\text { Salt Lake chron. . - }\end{array}$ & $\begin{array}{l}\text { I } 44 \quad 25.91 \\
9 \quad 5^{8} \\
47.24\end{array}$ & $\begin{array}{l}-01015.83 \\
+875.11\end{array}$ & $\begin{array}{lll}18 & 34 & 10.08 \\
18 & 5 & 52.35\end{array}$ & I 7.73 & 0.23 & 17.845 \\
\hline $\begin{array}{c}\text { August } 5 . \\
\text { Colorado Springs . }\end{array}$ & $\begin{array}{l}\text { Colo. Springs chron. } \\
\text { Salt Lake chron. . - }\end{array}$ & $\begin{array}{lll}18 & 46 & 45.80 \\
\text { I0 } & \text { I } & 5.27\end{array}$ & $\begin{array}{l}-01018.30 \\
+874.44\end{array}$ & $\begin{array}{lll}\text { I8 } & 36 & 27.50 \\
18 & 8 & 9.71\end{array}$ & 17.79 & & \\
\hline Salt Lake... & $\begin{array}{l}\text { Colo. Springs chron. } \\
\text { Salt Lake chron. - - }\end{array}$ & $\begin{array}{l}185125.60 \\
\text { Io } \quad 5 \quad 44.85\end{array}$ & $\begin{array}{l}-0 \text { 10 } 18.31 \\
+874.44\end{array}$ & $\begin{array}{lrr}18 & 41 & 7.29 \\
\text { I } 8 & 12 & 49.29\end{array}$ & 18.00 & 0.21 & 17.895 \\
\hline Salt Lake... & $\begin{array}{l}\text { Colo. Springs chron. } \\
\text { Salt Lake chron. : - }\end{array}$ & $\begin{array}{rrr}18 & 17 & 5.32 \\
9 & 31 & 23.72\end{array}$ & $\begin{array}{r}-01019.17 \\
+874.49\end{array}$ & $\begin{array}{l}\text { I } 646.15 \\
\text { I } 73828.21\end{array}$ & 17.94 & & \\
\hline Colorado Springs.\{ & $\begin{array}{l}\text { Colo. Springs chron. } \\
\text { Salt Lake chron. - . }\end{array}$ & $\begin{array}{lll}18 & 25 & 45 \cdot 3^{6} \\
9 & 40 & 3.97\end{array}$ & $\begin{array}{l}-0 \text { 10 19.18 } \\
+874.49\end{array}$ & $\begin{array}{l}18 \quad 1526.18 \\
\text { I } 747 \quad 846\end{array}$ & 17.72 & 0.22 & $17.83^{\circ}$ \\
\hline Colorado & ings east of Salt Lal & City . & & $-\quad-$ & oll $28^{\mathrm{m}}$ I & \pm 0.009 & \\
\hline
\end{tabular}


Mean Places of Stars for I873.0, used for Determination of Latitude of Colorado Springs, Colorado Territory.

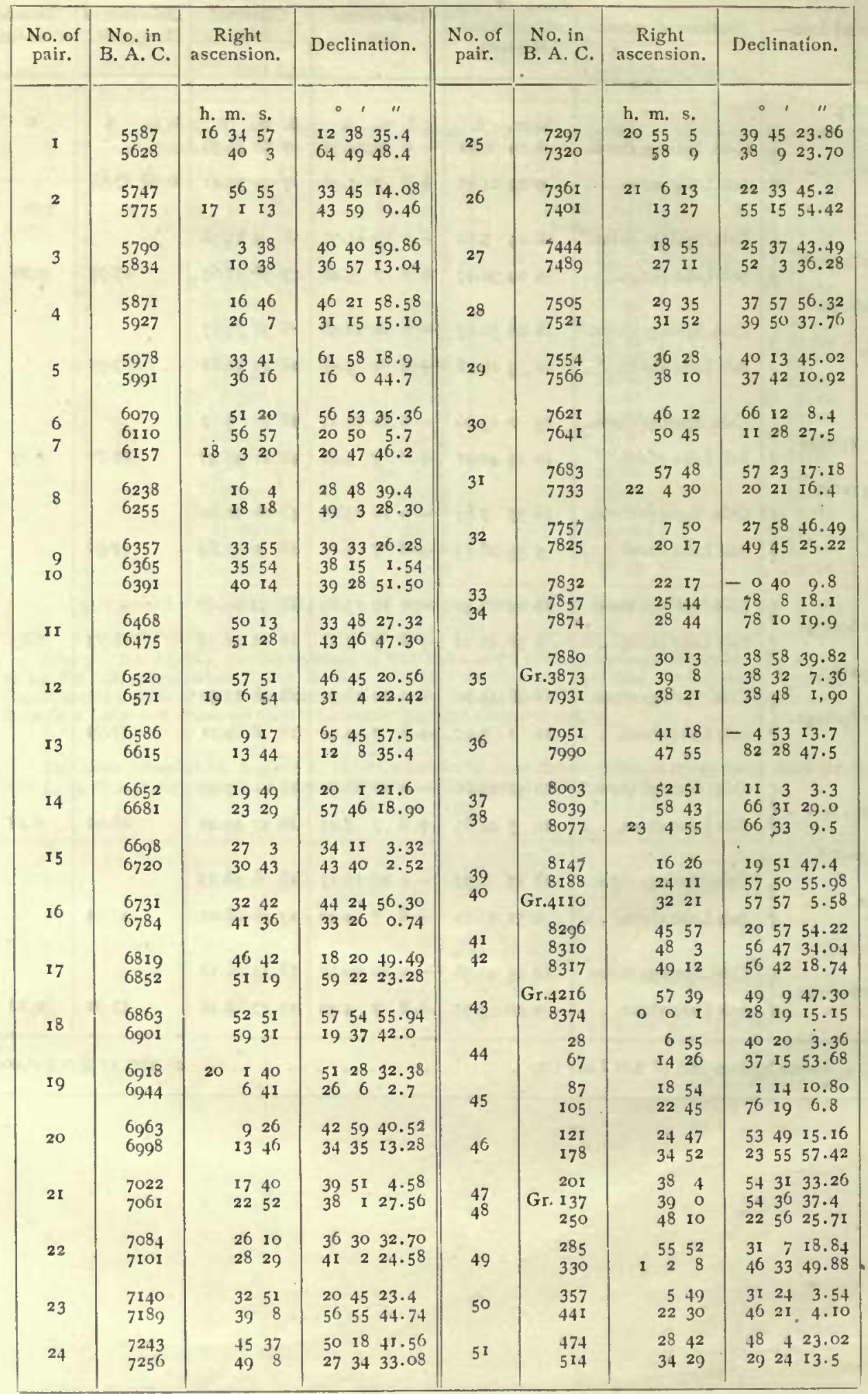


Observations for Latitude.-Stution, Colorado Springs.

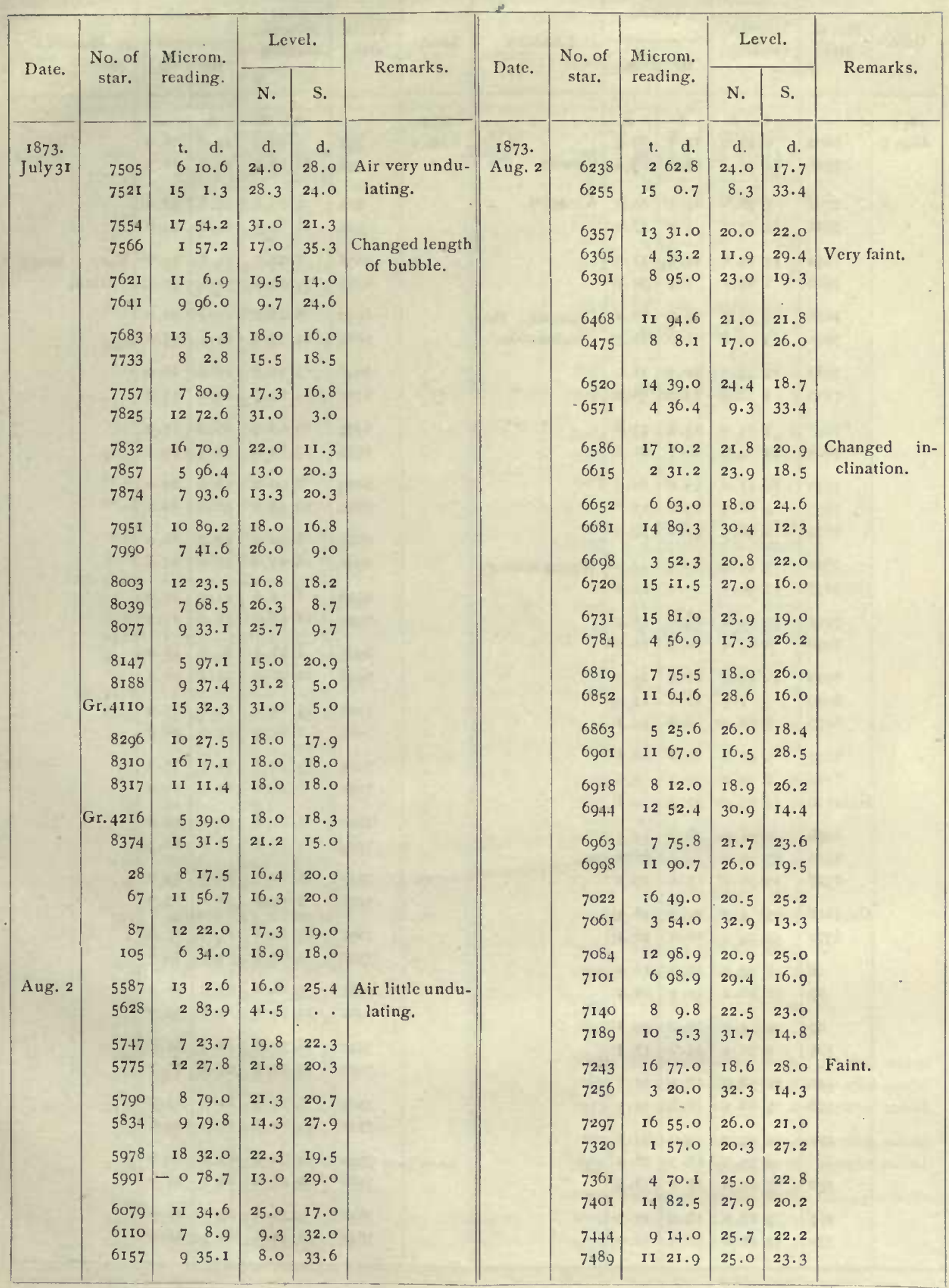


Observations for Latitude.-Station, Colorado Springs-Continned.

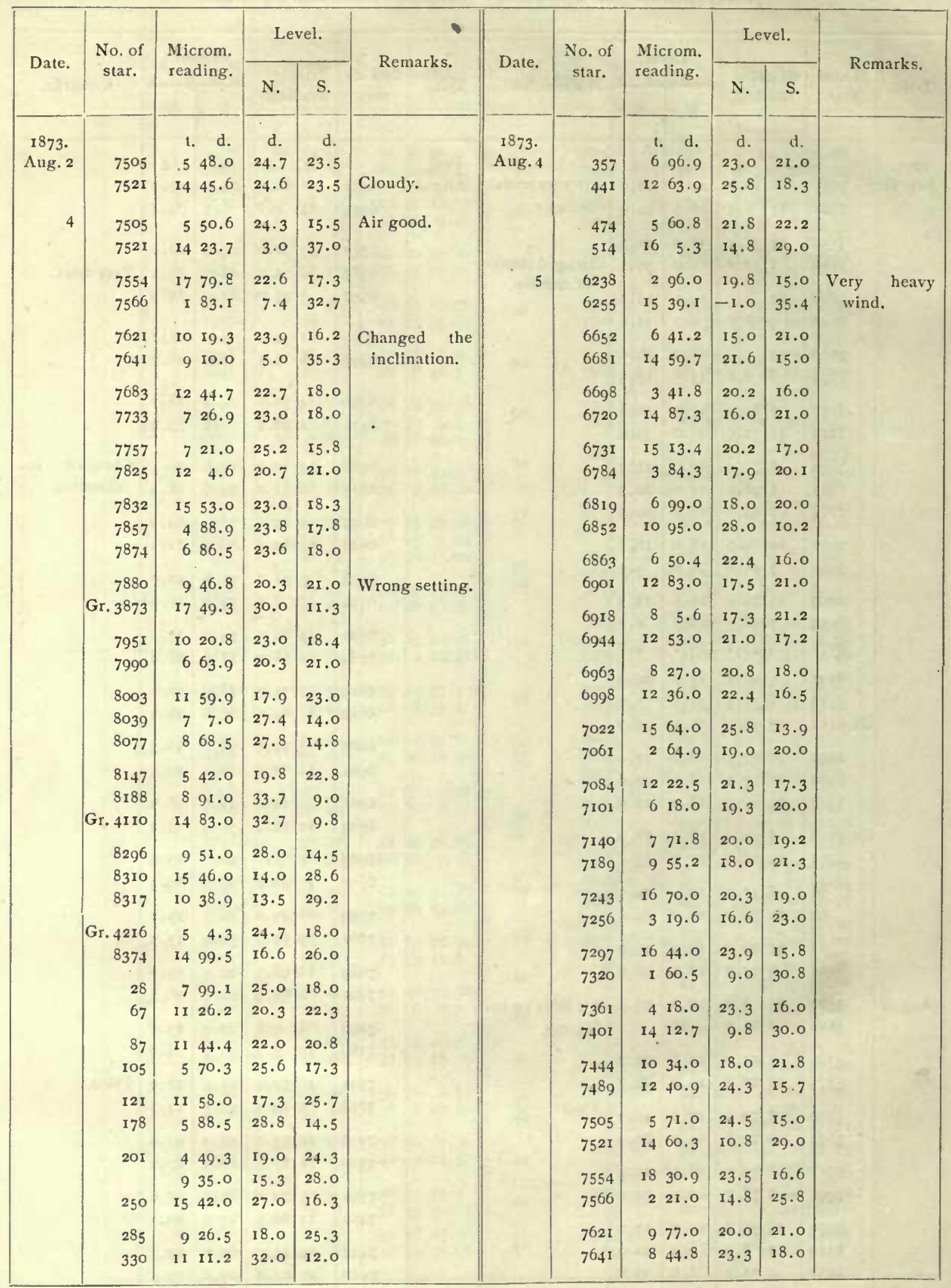


Observations for Latitude.-Station, Colorado Springs-Continued.

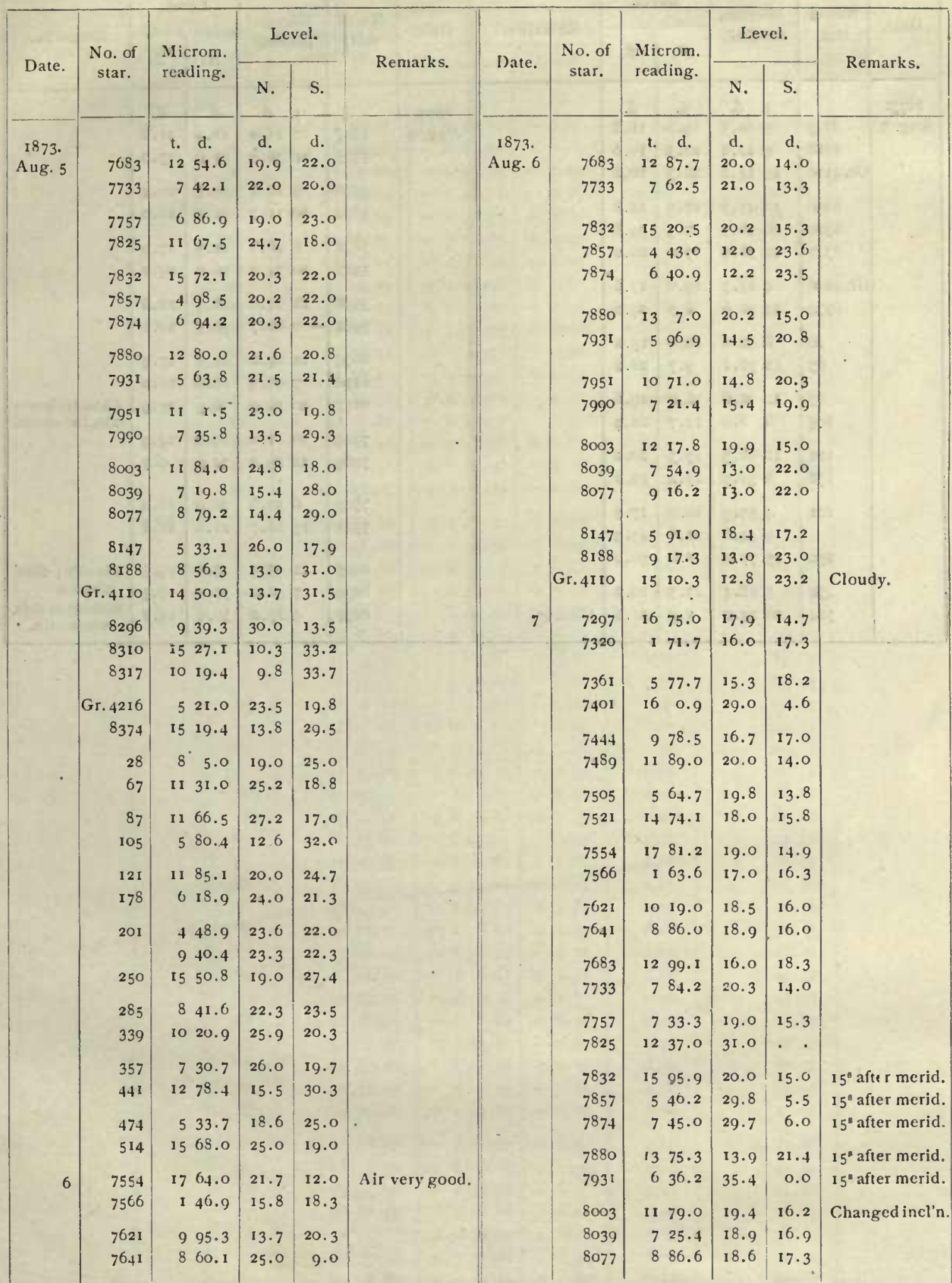


74

Observations for Latitude.-Station, Colorado Springs-Continued.

\begin{tabular}{|c|c|c|c|c|c|c|c|c|c|c|c|}
\hline \multirow{2}{*}{ Date. } & \multirow{2}{*}{$\begin{array}{l}\text { No. of } \\
\text { star: }\end{array}$} & \multirow{2}{*}{$\begin{array}{l}\text { Microm. } \\
\text { reading. }\end{array}$} & \multicolumn{2}{|c|}{ Level. } & \multirow{2}{*}{ Remarks. } & \multirow{2}{*}{ Date. } & \multirow{2}{*}{$\begin{array}{l}\text { No. of } \\
\text { star. }\end{array}$} & \multirow{2}{*}{$\begin{array}{l}\text { Microm. } \\
\text { reading. }\end{array}$} & \multicolumn{2}{|c|}{ Level. } & \multirow{2}{*}{ Remarks. } \\
\hline & & & N. & S. & & & & & N. & S. & \\
\hline \multirow{21}{*}{$\begin{array}{l}1873 . \\
\text { Aug. } 7\end{array}$} & & t. d. & d. & d. & & 1873. & & t. d. & d. & d. & \multirow{18}{*}{$\begin{array}{l}\text { Cloudy, heavy } \\
\text { rain,and storm. }\end{array}$} \\
\hline & 8147 & 580.5 & 19.8 & 36.5 & & Aug. 9 & 5747 & 725.0 & 18.0 & 21.6 & \\
\hline & 8188 & $92 \mathrm{I} .2$ & 20.3 & I6.6 & & & 5775 & 1236.0 & 27.0 & 13.0 & \\
\hline & Gr. 4110 & I5 14.2 & 20.3 & 16.6 & & & & 922.8 & & & \\
\hline & 82906 & I0 $45 \cdot 3$ & 22.0 & 14.8 & & & 5834 & 10 11.7 & 22.0 & 18.0 & \\
\hline & 8310 & 1646.5 & 17.3 & 19.8 & & & & & & & \\
\hline & 8317 & II 38.9 & 17.0 & 20.2 & & & 5871 & 846.8 & 22.3 & 17.9 & \\
\hline & Gr. 4216 & 485.3 & I9.6 & 17.6 & & & 5927 & I0 34.2 & 16.0 & 25.0 & \\
\hline & 8374 & I4 90.6 & 8.3 & 29.0 & & & $597^{8}$ & I 874.0 & 18.5 & 22.6 & \\
\hline & & & & & & & $599 \mathrm{I}$ & -062.7 & 30.3 & 10.8 & \\
\hline & $\begin{array}{l}28 \\
67\end{array}$ & 842.0 & 22.0 & 15.2 & & & 6079 & 1224.2 & 24.0 & 17.9 & \\
\hline & 67 & II 76.1 & 9.7 & 28.2 & & & 6110 & 790.9 & 16.0 & 26.0 & \\
\hline & 87 & II 92.6 & 18.0 & 19.6 & & & 6157 & Io 16.0 & 16.3 & 25.9 & \\
\hline & 105 & 68.0 & 14.7 & 22.9 & & & & I0 1.0 & & & \\
\hline & I 21 & II 88.3 & $17 \cdot 3$ & 20.2 & & & $\begin{array}{l}7444 \\
7480\end{array}$ & I2 23.2 & 19.0 & $24 \cdot 3$ & \\
\hline & 178 & 630.3 & 15.2 & 22.2 & & & 7409 & & 34.0 & 9.7 & \\
\hline & \multirow{2}{*}{$20 \mathrm{I}$} & 459.9 & 20.0 & 17.3 & & & 7505 & 614.6 & 26.0 & 16.0 & \\
\hline & & 954.6 & 21.8 & 15.9 & & & 7521 & 1528.7 & 21.8 & 21.8 & \\
\hline & 250 & 1577.0 & 1.0 & 36.0 & & & 8003 & II 93.5 & 25.0 & 19.5 & \multirow{3}{*}{$\begin{array}{l}\text { Cloudy; thro' } \\
\text { clouds. } \\
\text { Setting of mic. } \\
\text { uncertain. }\end{array}$} \\
\hline & 474 & 568.4 & 17.3 & 20.2 & & & 8039 & 752.0 & 30.0 & 14.5 & \\
\hline & 514 & 1624.0 & 5.9 & 31.8 & Cloudy. & & 8077 & 923.2 & 30.4 & 14.0 & \\
\hline
\end{tabular}


Computations for Latitude.-Station, Colorado Springs.

\begin{tabular}{|c|c|c|c|c|c|c|c|}
\hline \multirow{2}{*}{ Date. } & \multirow{2}{*}{$\begin{array}{l}\text { Number } \\
\text { of pair. }\end{array}$} & \multirow{2}{*}{$\begin{array}{l}\text { Half-sum of dec- } \\
\text { linations. }\end{array}$} & \multicolumn{3}{|c|}{ Corrections. } & \multirow{2}{*}{\multicolumn{2}{|c|}{ Latitude. }} \\
\hline & & & Mic. and ref. & Level. & Meridian. & & \\
\hline \multirow{18}{*}{$\begin{array}{c}\text { I873. } \\
\text { July } 3 I\end{array}$} & & $\circ, \quad 1$ & , " & " & $"$ & • , & " \\
\hline & 28 & $\begin{array}{lll}38 & 54 & \text { I9. } 62\end{array}$ & -436.72 & +0.08 & 0.00 & 38494 & 42.98 \\
\hline & 29 & $\begin{array}{ll}5^{8} & 0.46\end{array}$ & -816.16 & -2.36 & $0 . \infty 0$ & & 41.94 \\
\hline & 30 & $50 \mathrm{I} 8.86$ & -034.45 & -2.59 & 0.00 & & 41.82 \\
\hline & $3 I$ & 5219.10 & -236.11 & -0.27 & 0.00 & & 42.72 \\
\hline & 32 & $52 \quad 7.84$ & $-232: 75$ & +7.83 & 0.00 & 3 & $42.9^{2}$ \\
\hline & 33 & $44 \quad 6.75$ & +533.88 & +0.93 & 0.00 & & $4 \mathrm{~T} .56$ \\
\hline & 34 & $45 \quad 7.6 I$ & +432.34 & $+\mathrm{r} . \mathrm{or}$ & 0.00 & , & 40.96 \\
\hline & 36 & $47 \quad 49.29$ & +148.02 & +5.01 & 0.00 & . & 42.32 \\
\hline & 37 & $47 \quad 18.07$ & +221.36 & +4.45 & 0.00 & a & 43.88 \\
\hline & 38 & $4^{8} \quad 8.24$ & +130.23 & +4.01 & $0 . \infty 0$ & 4 & 42.48 \\
\hline & 39 & 5123.23 & - I 45.73 & $+5 \cdot 5^{8}$ & 0.00 & & 43.08 \\
\hline & 40 & 5427.92 & -450.57 & +5.52 & 0.00 & $=$ & 42.87 \\
\hline & $4 \mathrm{I}$ & 5245.50 & $-3 \quad 3.19$ & 0.00 & 0.00 & -1 & 42.31 \\
\hline & 42 & 50 $\quad 7.84$ & -026.07 & 0.00 & 0.00 & & 41.77 \\
\hline & 43 & 4432.40 & +58.36 & +1.63 & 0.00 & - & 42.39 \\
\hline & 44 & 4759.51 & +145.38 & -2.00 & 0.00 & $x$ & 42.89 \\
\hline & 45 & 4641.05 & +32.72 & -0.22 & 0.00 & & 43.55 \\
\hline \multirow[t]{27}{*}{ Aug. 2} & I & $\begin{array}{lll}38 & 44 & 17.10\end{array}$ & +516.52 & +8.83 & 0.00 & 38494 & 42.45 \\
\hline & 2 & $\begin{array}{ll}52 & 18.27\end{array}$ & $-236.6 \mathrm{I}$ & -0.27 & 0.00 & 4 & 41.39 \\
\hline & 3 & 4912.95 & +031.09 & -3.58 & 0.00 & $c^{2}$ & 40.46 \\
\hline & 5 & 5937.08 & -953.67 & -3.63 & 0.00 & - & 39.78 \\
\hline & 6 & $51 \quad 56.02$ & -212.26 & -4.03 & +0.30 & 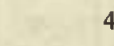 & $40 \% 3$ \\
\hline & 7 & 5046.29 & $-1 \quad 1.99$ & -4.84 & +0.30 & 1. & 39.76 \\
\hline & 8 & $469.43^{\circ}$ & -624.60 & -5.14 & 0.00 & 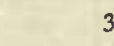 & 39.69 \\
\hline & 9 & $54 \mathrm{19.47}$ & -432.73 & $-5 \cdot 3^{6}$ & +0.04 & $x^{2}$ & $41 \cdot 42$ \\
\hline & Io & $52 \quad 2.04$ & -217.25 & -3.79 & 0.00 & 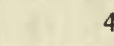 & 41.04 \\
\hline & II & 4742.42 & +20.07 & -2.69 & 0.00 & 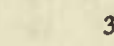 & 39.80 \\
\hline & I2 & 5456.64 & -511.50 & -5.06 & +0.04 & $=$ & 40.08 \\
\hline & I3 & 5720.68 & -739.54 & +1.73 & 0.00 & 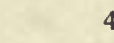 & $42.9 \pi$ \\
\hline & I4 & $53 \quad 53.73$ & -416.72 & +3.16 & 0.00 & $t=$ & 40.17 \\
\hline & I5 & $55 \quad 37.74$ & -60.15 & +2.69 & 0.00 & 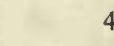 & 40.28 \\
\hline & I6 & 5533.12 & -549.24 & -1.10 & 0.00 & $x^{2}$ & 42.78 \\
\hline & I 7 & 5140.50 & $\begin{array}{ll}-2 & 0.84\end{array}$ & +1.26 & 0.00 & pent & 40.92 \\
\hline & 18 & $46 \quad 23.19$ & +319.28 & -1.21 & 0.00 & $a^{2}$ & 41.26 \\
\hline & I9 & 4721.81 & +216.82 & +2.53 & 0.00 & 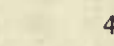 & 41.16 \\
\hline & 20 & 4731.17 & +28.90 & +1.26 & 0.00 & $y^{2}$ & $4 \mathrm{I} \cdot 33$ \\
\hline & $2 \mathrm{I}$ & 5620.23 & -642.34 & +4.12 & 0.00 & 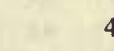 & 42.01 \\
\hline & 22 & 4632.70 & $+36.4 \mathrm{I}$ & +2.31 & 0.00 & $x^{2}$ & 41.42 \\
\hline & 23 & 5037.75 & $\begin{array}{ll}-\mathrm{I} & 0.74\end{array}$ & $+4 \cdot 5 t$ & 0.00 & a & 41.52 \\
\hline & 24 & 5641.06 & -71.60 & +2.36 & 0.00 & & 41.80 \\
\hline & 25 & 5727.43 & $-745.4 \mathrm{I}$ & -0.55 & 0.00 & 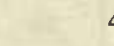 & 41.47 \\
\hline & 26 & 5453.13 & -514.55 & +2.72 & 0.00 & $=$ & 41.30 \\
\hline & 27 & 5043.09 & - I 4.59 & +1.43 & 0.00 & te & 39.93 \\
\hline & 28 & 5420.25 & -438.88 & +0.63 & 0.00 & $x^{2}$ & 42.00 \\
\hline & 28 & $3^{8} .54 \quad 20.87$ & -431.26 & -6.93 & 0.00 & 3849 & 42.68 \\
\hline & 29 & $5^{8} \quad$ I. 72 & -816.08 & $-5 \cdot 50$ & 0.00 & & 40.14 \\
\hline & 30 & $50 \quad 19.96$ & -033.94 & $-6.2 \mathrm{I}$ & 0.00 & & $39.8 \mathrm{I}$ \\
\hline & $3 \mathrm{I}$ & 5220.47 & -240.89 & +2.67 & 0.00 & & 42.25 \\
\hline & 32 & $52 \quad 9.08$ & -230.25 & +2.50 & 0.00 & & $4 \pi \cdot 33$ \\
\hline & 33 & $44 \quad 7.72$ & +530.66 & +2.94 & 0.00 & & $4 \mathrm{r} \cdot 32$ \\
\hline & 34 & $45 \quad 8.56$ & +429.25 & +2.83 & 0.00 & & 40.64 \\
\hline
\end{tabular}


Computation for Latitude._Station; Colorado Springs-Continued.

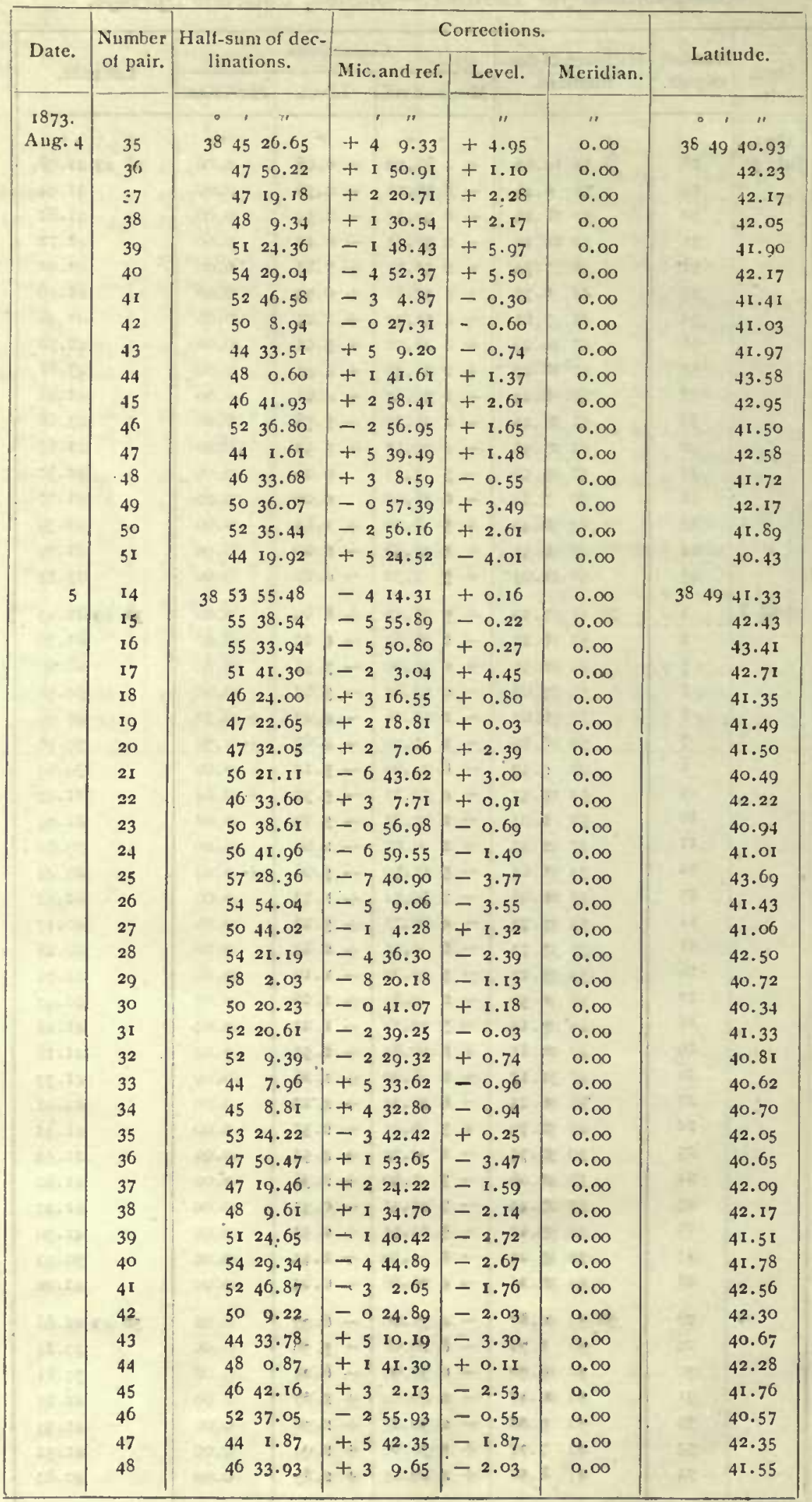


Computations for Latitude.-Station, Colorado Springs-Continued

\begin{tabular}{|c|c|c|c|c|c|c|c|}
\hline \multirow{2}{*}{ Date. } & \multirow{2}{*}{$\begin{array}{l}\text { Number } \\
\text { of pair. }\end{array}$} & \multirow{2}{*}{\multicolumn{2}{|c|}{$\begin{array}{l}\text { Half-sum of dee- } \\
\text { linations. }\end{array}$}} & \multicolumn{3}{|c|}{ Corrections. } & \multirow{2}{*}{ Latitude. } \\
\hline & & & & Mic. and ref. & Level. & Meridian. & \\
\hline \multirow{4}{*}{$\begin{array}{l}\text { I } 873 . \\
\text { Aug. } 5\end{array}$} & \multirow[b]{2}{*}{49} & \multicolumn{2}{|c|}{$\circ, "$} & , $"$ & " & $"$ & \multirow{2}{*}{ 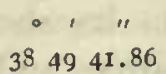 } \\
\hline & & $3^{8} 5$ & 5036.36 & $-055.7 \mathrm{I}$ & $+1.2 \mathrm{I}$ & 0.00 & \\
\hline & 50 & & 5235.67 & -250.17 & -2.34 & 0.00 & $\begin{array}{r}384941.86 \\
43.16\end{array}$ \\
\hline & 51 & & 4420.15 & +521.35 & -0.11 & 0.00 & 41.39 \\
\hline \multirow[t]{11}{*}{6} & 29 & \multicolumn{2}{|c|}{$\begin{array}{lll}3^{8} & 5^{8} & 2.34\end{array}$} & $-822.4 I$ & +1.98 & 0.00 & \multirow{2}{*}{$\begin{array}{rr}3849 & 41.01 \\
41.09\end{array}$} \\
\hline & 30 & & 5020.51 & -042.00 & $+2.5^{8}$ & 0.00 & \\
\hline & 31 & & 5220.90 & $-2+3.18$ & +3.77 & 0.00 & 41.49 \\
\hline & 33 & & $44 \quad 8.20$ & +534.84 & $-I .84$ & 0.00 & $4 I .20$ \\
\hline & 34 & & $45 \quad 9.05$ & +433.33 & - I. 76 & 0.00 & 40.62 \\
\hline & 35 & & 5324.54 & -340.63 & -0.30 & 0.00 & $43.6 \mathrm{I}$ \\
\hline & $3^{6}$ & & 4750.71 & +154.85 & -2.75 & $0 . \infty 0$ & +2.81 \\
\hline & 37 & & $47 \quad 19.75$ & +223.82 & -1.13 & 0.00 & 42.44 \\
\hline & $3^{8}$ & & $4^{8} \quad 9.89$ & +133.71 & -1.13 & 0.00 & 42.47 \\
\hline & 39 & & 5124.94 & -141.39 & $-2 \cdot+2$ & 0.00 & $4 I . I 3$ \\
\hline & 40 & & $54 \quad 29.62$ & -445.64 & -2.53 & 0.00 & $41 \cdot 45$ \\
\hline 7 & 25 & $3^{8} 5$ & 5728.97 & -747.05 & -0.52 & 0,00 & $38 \quad 49 \quad 41.40$ \\
\hline & 26 & & 5454.62 & -517.90 & +3.16 & 0.00 & 39.88 \\
\hline & 27 & & 5044.96 & - I $5.4 \mathrm{I}$ & +1.57 & 0.00 & 41.12 \\
\hline & 28 & & 5421.81 & -442.15 & +2.23 & 0.00 & 41.89 \\
\hline & 29 & & $5^{8} \quad 2.66$ & -822.63 & $+\mathrm{r} .32$ & 0.00 & $41 \cdot 35$ \\
\hline & 30 & & 5020.77 & -041.32 & $+\mathrm{I} .48$ & 0.00 & 40.93 \\
\hline & $3 \mathbf{I}$ & & 5221.20 & $-239: 99$ & +1.10 & 0.00 & 42.31 \\
\hline & 32 & & 5210.01 & -246.49 & +9.54 & 0.00 & 43.06 \\
\hline & 33 & & 448.46 & +526.19 & +8.06 & 0.00 & 42.71 \\
\hline & 34 & & $45 \quad 9.29$ & +429.41 & +7.89 & 0.00 & 41.59 \\
\hline & 35 & & 5324.86 & -349.63 & +7.70 & +0.06 & 42.99 \\
\hline & 37 & & 4720.02 & +230.93 & +1.43 & 0.00 & $42 \cdot 38$ \\
\hline & 38 & & 48 10. 18 & +130.85 & $+I .24$ & 0.00 & 42.27 \\
\hline & 39 & & $51 \quad 25.22$ & - I. 45.86 & +1.84 & 0.00 & 41,20 \\
\hline & 40 & & 5429.91 & -450.11 & +1.84 & 0.00 & 41.64 \\
\hline & 41 & & 5247.44 & -36.79 & +1.29 & 0.00 & 41.94 \\
\hline & $4^{2}$ & & $50 \quad 9.79$ & -029.08 & +1.10 & 0.00 & 41.81 \\
\hline & 43 & & $4434 \cdot 35$ & $+5 \mathrm{I2.31}$ & -5.14 & 0.00 & 41.52 \\
\hline & 44 & & $4^{8} \quad 1.43$ & +143.79 & -3.22 & 0.00 & 42.00 \\
\hline & 45 & & 4642.62 & +31.67 & -2.69 & 0.00 & 41.60 \\
\hline & 46 & & $5237 \cdot 52$ & -253.38 & -2.72 & 0.00 & $4 \pi \cdot 4^{2}$ \\
\hline & 47 & & $\begin{array}{ll}44 & 2.3^{8}\end{array}$ & +547.07 & -8.88 & 0.00 & 40.57 \\
\hline & 48 & & $46 \quad 34.45$ & +313.37 & -8.00 & 0.00 & 39.82 \\
\hline & $5 \mathrm{I}$ & & 4420.60 & +527.95 & -7.92 & 0.00 & 40.63 \\
\hline 9 & 2 & 385 & $52 \quad 19.24$ & -238.76 & +2.86 & 0.00 & $3^{8} 4943 \cdot 34$ \\
\hline & 3 & & 4913.96 & +027.62 & +0.85 & 0.00 & 42.43 \\
\hline & 4 & & $48 \quad 44.25$ & +058.23 & -1.26 & 0.00 & 41.22 \\
\hline & 5 & & 5938.12 & $\begin{array}{ll}-10 & 1.74\end{array}$ & +4.23 & 0.00 & 40.61 \\
\hline & 6 & & $51 \quad 57.25$ & -214.62 & -1.07 & 0.00 & $41 \cdot 56$ \\
\hline & 7 & & $5047.5 \mathrm{I}$. & $-1 \quad 4.69$ & -0.96 & 0.00 & 41.86 \\
\hline & 27 & & $5045.2 \mathrm{I}$ & -19.03 & +5.23 & 0.00 & $4 I \cdot+r$ \\
\hline & 28 & 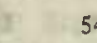 & 5422.43 & -444.00 & +2.86 & 0.00 & 41.29 \\
\hline & 37 & 4 & 4720.56 & +217.17 & +5.77 & +0.19 & 43.88 \\
\hline & 38 & 4 & $48 \quad 10.77$ & +226.10 & +6.02 & +0.19 & 43.28 \\
\hline
\end{tabular}


The observations for latitude were made under very unfavorable circumstances. Undulations in the atmosphere, heavy winds, and great changes in temperature constantly affected the instrument.

It is customary in this office to select for latitude only thirty-five pairs of stars, which have to be observed on five different nights. But the observer is dependent upon the weather, and is frequently disappointed, although after waiting I preferred to select a greater number of pairs, believing that the final result of a latitude is better when depending upon various star-places, giving the probability that the errors resulting from the declination-places of the stars will more nearly compensate each other, and that this part of the probable error of the final result will come within that resulting from observation alone.

The mean latitnde is obtained by taking the mean of all single results. For the different days the mean latitude is found to be as follows:

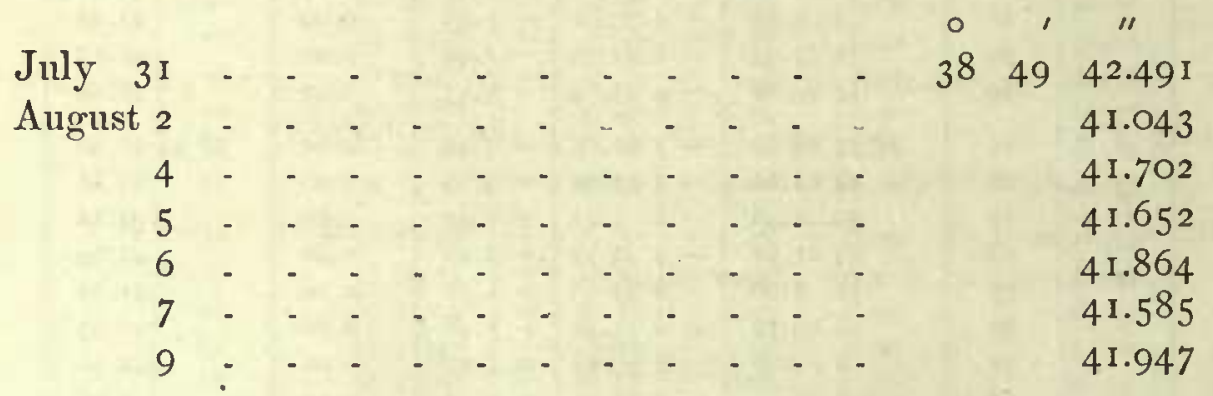

slowing a great difference between the first and second day, arising from the disturbed condition of the air.

The probable error of one observation is,

$$
\varepsilon_{\circ}= \pm 0.6745 \wedge \sqrt{\frac{\overline{\Sigma(v)^{2}}}{n-1}}
$$

where $v$ is the difference between the mean results and the single resilts and $n$ the number of observations; therefore the probable error of the mean result is,

$$
\varepsilon_{r}= \pm 0.6745 \wedge \sqrt{\frac{\sum(v)^{2}}{n(n-I)}}
$$

If it is proper to place all the observations in the final result with the same weight (as in determining the longitude of a station from different niglits' work) the formula should be used in this way; but in determining the latitude of a station, every single result obtained also depends upon the places of the stars forming the different pairs. It is certainly wrong to determine the probable error of the latitude-result by this formula, (it would give, for latitude of Colorado Springs, a probable erxor less than o".or,) 
though it is frequently done. If nearly the same number of stars are observed every night under the same conditions, I should prefer to determine the probable error of the final result after the manner of Mr. John H. Clark.

Let-

Probable error of one pair of stars, including constant errors of zenith-telescope observations _ _ _ _ _ _ _ $=\varepsilon_{p}$

Probable error of one observation _ _ _ _ - - - $_{-}=\varepsilon_{\mathrm{o}}$

Number of pairs used at the station _ _ _ _ . - $=m$

Number of observations _ . . . . . . . . . . . $=n$

then probable eiror of the final result,

$$
\varepsilon_{r}= \pm \sqrt{\frac{\varepsilon_{p}^{2}}{m}+\frac{\varepsilon_{o}{ }^{2}}{n}}
$$

The formula shows that if the stars used are not very good, it is then better to select a larger number of pairs of stars, giving the probability that the final result will be more independent of the declinations.

From all the observations of pairs of stars observed on three or more nights, I find the probable error of one observation-

and that of the final result,

$$
\varepsilon_{\mathrm{o}}= \pm \mathrm{o}^{\prime \prime} .428
$$

$$
\varepsilon_{r}= \pm 0^{\prime \prime} .035
$$

T'aking the value for $\varepsilon_{p}$, found by Maj. C. B. Comstock, of the United States Lake Survey, for stars taken from Professor Safford's Catalogue for 981 Stars, $\pm 0^{\prime \prime} \cdot 53$, the probable uncertainty of the final result will be, $\pm 0^{\prime \prime} .082$.

Resulting Astronomical Co-ordinates for the Astronomical Monument at Colorado Springs, Colorado Territory, using, for the longitude of Washington and Salt Lake, the same data as in Clark's report.

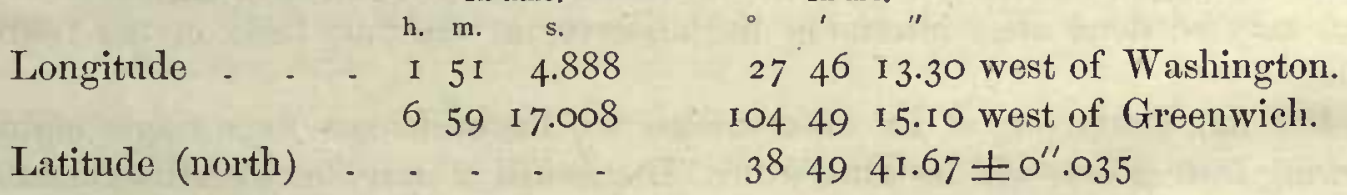

Respectfully submitted.

First Lieut. Geo. M. Wheeler,

DR. F. KAMPF, Civilian Astronomical Assistant.

Corps of Engineers, in charge. 


\section{United States Enginefr Office, Geographical and Geological Explorations and Surveys \\ West of the iooth Meridian, May I, I 873 .}

Memorandum of instruetions for conducting observations for longitude and latitude at a main or primary astronomical station for the field-8rason of 1873 .

The fixed observatory, with which connection will be made, is in Temple Square, Salt Lake City, Utah, and in charge of Assistant John H. Clark. Every observer is responsible for the receiving of the signals from the connecting-stations for certain reductions of results, as full as possible, and will follow such other instructions that may, from time to time, be given. This station will be changed during the season, probably in the month of Angust, to the United States engineer observatory, to be constructed at Ogden, Utah.

\section{I.-Observations for Longitude.}

In carrying on the astronomical campaign, preference will be given to the longitude-results for those nights that are clear at both stations; that is, the observers in charge, after having completed the necessary arrangements for the observations and exchange of signals, will, from this time on, exchange signals each night that shall be fair at the two stations, unless unavoidable difficulties arise to prevent.

It will be carefully observed that weather-signals are sent by telegraph each evening about $6 \mathrm{p}$. m. while the exchanges are going on and until the full series at a station shall have been completed. In case of uncertainty as to the possibility for observing for any one night, this may be indicated in the first dispatch; and subsequent dispateh or dispatches, between this time and 10 p. m., shall determine whether exchanges shall be made that night or not.

Some convenient and concise form will be adopted for the weather-signals, and in no case must there be a failure as regards forwarding them.

It may be admissible, contingent upon the stage of the prosecution of the work, should the weather at Salt Lake at about $6 \mathrm{p}$. m. look unfavorable, that the observer at the distant station should at once conclude to take that night for latitude-work, which may be done after informing the observer at the Salt Lake or the receiving station.

For the conducting of the observations for the exchanges for a single niglit, the following instructions will be adhered to: The transit of stars for time-determinations, consisting of not less than three time and two circumpolar stars in each position of the instrument, both before and after the transmission of the signals, will be taken. Of course, should the night be sufficiently clear, so that it seems possible to make the time-determinations at both stations, by the modification of the above, so that at one or both of the stations the transits of star's may be made entirely before or entirely after the sending of the signals, the strict following-out of the above is not necessary.

These instructions are made with the understanding that a twenty-six or thirty- 
two inch Wiirdemann instrument is used, with recording-apparatus in shape of a chronograph or register.

The time set for the transmission of signals should be as near $9.30 \mathrm{p}$. m. local time of the Salt Lake meridian as possible; and great care should be taken that both observers shall be on hand simultaneously, so that as little delay as possible shall ensue in the use of the telegraph-wires.

In the transmission of signals the record is to be made upon the chronograph or register at the two places over a space of five minutes in time, the connecting or Salt Lake station sending for the first five minutes and the distant station receiving, and vice versa.

In addition to these, which may be known as the chronograph-signals, arbitrary signals will be sent, by the use of a.break-circuit key, at about ten seconds apart, at fractional parts of a second, making a series of thirty-one arbitrary signals during the five minutes.

In order that the observer at a station may conclude that he has accomplished six nights of first-class observations, it becomes necessary that there should be an exchange of the approximate results. Each observer will therefore send to the other, upon each subsequent night or as soon thereafter as practicable, the approximate error of his chronometer and the mean of seven arbitrary signals, sent and received, selected from the middle of the set of thirty-one. This may be concisely expressed in a telegram.

For the full satisfaction of an observer at the distant station, such further computation shall be sent and received as shall seem necessary to a clear understanding of the case; as an observer will be held responsible slould he leave the station and go to another before he is certain that the results upon final computation would prove satisfactory.

\section{2.-Observations for Latitude.}

These will be conducted through five complete and clear nights, so that there shall not be less than 175 pairs of observations upon 35 separate and distinct pairs of stars, each pair of which observations shall give a first-class result.

These instructions are furnished to the observers with a view to their clearly understanding the class of results intended, and will always be carried out, unless unforeseen difficulties arise, in which event, as the observers will not hereafter be within speedy communication of these headquarters, it will be necessary for them to adopt immaterial modifications upon their own responsibility. It is, of course, understood that each observer is responsible for and receives the credit of his own work.

A report will be made by each observer, at intervals not exceeding fifteen days, of the work under his charge.

A full daily journal will be kept by each observer. Great care will be taken as to the character of the record upon this journal, which should be clear and explicit. The position of the station in reference to surrounding natural objects should be clearly described, and imperishable meridian-marks firmly planted. When possible, a special survey and plat will be made. 
The day following an exchange of the chronographic record of time and excliangesignals, said signals will be carofully copied into a record-book, which, like all other records, must be made in duplicate.

It is intended that computations in the field sliall be carried on to suel an extent as circumstances may permit.

The time that can be allotted to each of these stations, in order to accomplish the expected results for the season, is from twenty to twenty-five nights; and as obscrvations are required only for eleven nights, the remaining interval shonld be employed in computation.

The order of sequence for an astronomical report is furnished herewitl, and the records and journal will be so kept as to comprise all the data necessary for the full expression of results in accorlance therewith. .

Official :

GEO. M. WHEELER,

First Lieutenant Corps of Engineers, in charge. 





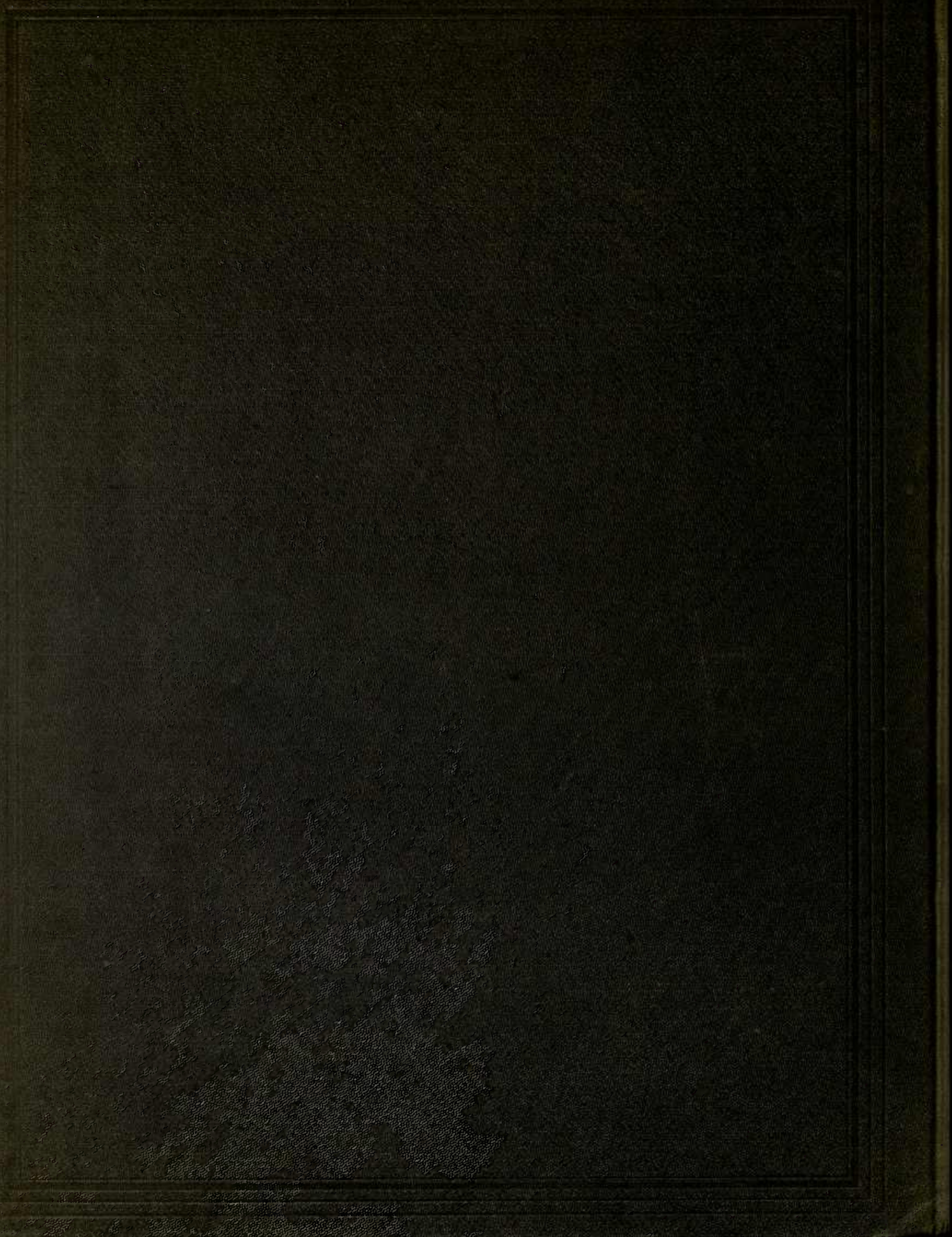

